University of Louisville

ThinkIR: The University of Louisville's Institutional Repository

Electronic Theses and Dissertations

$12-2007$

\title{
Therapeutic targeting of tumor associated macrophages.
}

Stephanie Kaye Watkins 1981-

University of Louisville

Follow this and additional works at: https://ir.library.louisville.edu/etd

\section{Recommended Citation}

Watkins, Stephanie Kaye 1981-, "Therapeutic targeting of tumor associated macrophages." (2007). Electronic Theses and Dissertations. Paper 1535.

https://doi.org/10.18297/etd/1535

This Doctoral Dissertation is brought to you for free and open access by ThinkIR: The University of Louisville's Institutional Repository. It has been accepted for inclusion in Electronic Theses and Dissertations by an authorized administrator of ThinkIR: The University of Louisville's Institutional Repository. This title appears here courtesy of the author, who has retained all other copyrights. For more information, please contact thinkir@louisville.edu. 
THERAPEUTIC TARGETING OF TUMOR ASSOCIATED MACROPHAGES

By

Stephanie Kaye Watkins

B.A. Bellarmine University, Louisville, KY 2003

M.S. University of Louisville, Louisville, KY 2005

\author{
A Dissertation \\ Submitted to the Faculty of the \\ Graduate School of the University of Louisville \\ in Partial Fulfillment of the Requirements \\ for the Degree of
}

Doctor of Philosophy

Department of Microbiology and Immunology

University of Louisville

Louisville, KY

December 2007 
Copyright 2007 by Stephanie K. Watkins

All rights reserved 
Stephanie Kaye Watkins

B.A. Bellarmine University, Louisville, KY 2003

M.S. University of Louisville, Louisville, KY 2005

A Dissertation Approved on

September 20, 2007

by the following Dissertation Committee:

Dissertation Director 


\section{DEDICATION}

I would like to dedicate this dissertation to my family: My parents, Ed Sermon and Margie Haines, whom have provided both the loving and financial support that have guided me through all of my scholastic ambitions, to my husband, Keith for his constant support and understanding, and to my son Tyler, whom has taught me more than one could ever imagine. 


\section{ACKNOWLEDGMENTS}

First, I would like to thank my advisor Dr. Robert D. Stout for all of the opportunities which he has provided me over the last four years. I am grateful to him for letting me work on this exciting project, for his guidance, continuous support and the opportunities to present my work at national and international meetings. He allowed me freedom to learn independence, yet was always available when help was needed or wanted, in spite of his many departmental obligations, and most importantly, was patient with me. One could not ask for a better mentor and friend. I'm eternally grateful for his guidance both in the laboratory and in life.

I would like to say thanks to the many current members, Joe, Kim, Duygu, Lihau, Qun, Xinyan, and Bing and past members, Bharati, Jeff, Melanie, Illya, Chenchang, Katherine, and Lata, of the Stout and Suttles labs, whom made working there interesting and enjoyable. Thanks to everyone for your friendship, I had fun working with you all! I will especially miss Joe, who always had the right thing to say or weird little noise to make which could immediately cheer me up when frustrations were high.

I would also like to express thanks to the members of my committee, Drs. Jill Suttles, Jun Yan, Robert Mitchel, Hari Bodduluri, and Nejat Egilmez for all suggestions, techincal help, comments, and criticisms. Thanks to the Department of Microbiology and Immunology and IPIBS program for the opportunity to work here at the University of Louisville. I would also like to acknowledge past and present fellow grad students for 
their friendship and sharing their experiences both good and bad, so that we all had to opportunity to learn from them, especially Joe, Jennifer, and Deanna.

Last but not least, I would like to thank my family - my husband Keith, my son Tyler and dog Sadie, my parents Margie and Matt Haines and Ed and Sheila Sermon, my other parents and sister Ross, Linda and Hayley Watkins, our Louisville family Ross and Linda Sherer, and Jennifer, Mike and Rachel Littrell and those we consider family Maggie and Brian Harlow, Brad and Debbie Gianulis, and the entire Turi family. Thank you all for your kindness and generosities, I would not have been able to work on and prepare this dissertation without your help and support. 


\begin{abstract}
THERAPEUTIC TARGETING OF TUMOR ASSOCIATED MACROPHAGES

Stephanie K. Watkins
\end{abstract}

September 20, 2007

Previous studies on the mechanistic induction of anti-tumor responses by IL-12 cytokine therapy have focused on the adaptive immune response, specifically the activation NK cells and T cells as the primary targets of IL-12 treatment. In contrast, little attention has been given to the potential role of macrophages in the initiation of antitumor responses by IL-12 therapy despite reports that macrophages play a major role in promoting tumor growth and metastasis and in suppressing anti-tumor immune responses. Based on the functional adaptivity hypothesis, which is the concept that macrophages functionally adapt, rather than differentiate into specific mature subsets, in response to environmental stimuli, we hypothesized that tumor infiltrating (TIMs) as well as tumorassociated macrophages (TAMs) could be converted from tumor supportive activities to pro-immunogenic activities by IL-12 therapy. We examined the functional activities displayed by TIMs and TAMs after treatment with IL-12. Our data demonstrate (1) that tumor cells and tumor exosomes activate TIMs and TAMs by cell-contact dependent mechanisms involving ligation of CD40 and/or NKG2D, (2) that IL-12 treatment both in vivo and in vitro induces a rapid reduction of tumor supportive activities and a concomitant increase in pro-inflammatory activities in TIMs as well as TAMs, (3) that 
IL-12 induces a rapid release of cytoplasmic IL-15 from the in situ activated tumor associated macrophages and (4) the release of IL-15 in essential to the recruitment of lymphocytes to the tumor and the metastatic lung, and to the destruction of the tumor and clearance of metastasis. It is concluded that macrophages in the tumor environment are activated and functionally modulated by the tumor. TIMs and TAMs respond to IL-12 treatment by rapidly converting from suppressive, tumor supportive activities to inflammatory, pro-immunogenic activities. Tumor associated macrophages thus are a critical target of IL-12 therapy and may orchestrate the subsequent NK and T cell cytotoxic response against the tumor. 


\section{TABLE OF CONTENTS}

\section{PAGE}

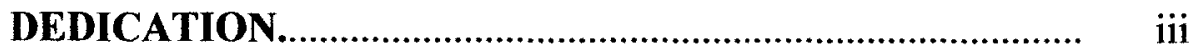

ACKNOWLEDGMENTS................................................... iv

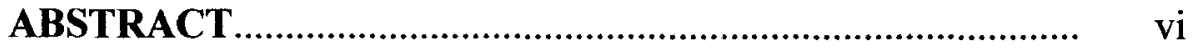

LIST OF FIGURES...............................................................

INTRODUCTION .................................................................

Macrophage Activation and Plasticity ............................... 1

Tumor Associated Macrophages........................................ 5

TAM Activation............................................................. 10

TAM Plasticity and Cytokine Therapy............................. 12

Hypothesis, Significance, Summary................................... 15

MATERIALS AND METHODS............................................ 18

RESULTS

Tumor Associated Macrophage Phenotype....................... 25

IL-12 Treatment in vitro Alters TAM Phenotype.............. 27

IL-12 Treatment in vivo Alters TIM/TAM Phenotype..... 33

IL-12 Induces TAM IL-15 Release................................... 37

TAMs Aid in IL-12 Induced Immuno-Activation............. 39

Macrophages Activated by Tumor Exosomes.................. 47

Tumors Activate Macs via CD40/NKG2D Ligation......... 55

CD40 Essential for Metastatic Growth............................ 63

TAMs Stimulate Tumor Cells............................................ 68

Table 1. Tumor-macrophage interactions.............. 73 
DISCUSSION

Conclusions.

REFERENCE LIST

CURRICULUM VITAE 


\section{LIST OF FIGURES}

FIGURE

PAGE

1. Macrophage functional diversity................................................ 2

2. Tumor induced macrophage function........................................

3. IL-12 modulation of TAM function......................................... 17

4. TAM display the anti-inflammatory "suppressor"phenotype similar to tumor infiltrating macropahges.................................... 26

5. IFN $\gamma$ restores $\mathrm{CD} 11 \mathrm{~b}^{\text {hi }} \mathrm{Gr}-1{ }^{-}$phenotype.................................. 28

6. IFN $\gamma$ reduces TAM pro-tumorigenic cytokine production in response to LPS stimulation....................................................... 29

7. IL-12 impact on TAM cytokine gene expression..................... 31

8. In vitro treatment alters cytokine gene expression................ 32

9. IL-12 changes the pattern of cytokine production in vitro in response to LPS stimulation........................................................

10. In vivo IL-12 treatment induces a rapid shift in the cytokine profile that is sustained for at least 5 days

11. In vivo injection of IL-12 induces a rapid shift to pro-inflammatory functions systemically.

12. IL-12 injection induces a decrease in pro-tumorigenic and an increase in pro-immunogenic cytokine genes.

13. TAMs release IL-15 in vivo in response to IL-12 treatment..

14. TAM cytoplasmic IL-15 is released rapidly as a bolus within 2-3 hours and is replenished by $24 \mathrm{hrs}$ after IL-12 treatment...... 
15. Neutralization of IL-15 reduces $T$ cell infiltration in response to IL-12

16. Neutralizing IL-15 prevents IL-12 induced tumor destruction.

17. Neutralization of IL-15 reduces $T$ cell infiltration into metastatic tissue in response to IL-12 treatment.

18. IL-15 neutralization prevented the clearance of tumor metastasis in the lung.

19. IL-12 treatment prevents the formation or induces the breakdown of the major tumor blood vessel, which is prevented upon neutralization of IL-15

20. Macrophages in beige mice respond to IL-12 treatment

21. Depleting NK cells does not inhibit $\mathrm{T}$ cell infiltration in response to IL-12 induced IL-15 release.

22. TAMs are actively producing cytokines upon ex vivo analysis. $\quad 53$

23. Unfiltered tumor supernatant activates macrophages.

24. Tumor exosomes activate macrophages

25. Tumor cells express CD40L and Rae- 1 and TAMs but not normal macrophages express elevated levels of surface CD40 and NKG2D receptors.

26. TAMs express elevated NKG2D mRNA gene expression........

27. CD40 and NKG2D are involved in macrophage activation by tumor exosomes

28. Blockade of either NKG2D or CD40 ligation interferes with exosome activation of macrophages

29. Anti-NKG2D Ab specifically blocks macrophage activation by exosome NKG2D ligation as shown rmNKG2D-Fc inhibited the ability of the blocking $\mathrm{Ab}$ to prevent cytokine production........

30. CD40 and NKG2D are involved in macrophage activation by tumor exosomes 
31. Blocking Abs to NKG2D and CD154 do not inhibit macrophage cytokine production in response to LPS stimulation.. $\quad 65$

32. Cytokine secretion is decreased in TAMs from CD40-/- tumor bearing mice.

33. Tumor activated macrophage $\mathrm{IL}-15$ release is blocked in the absence of CD40 and/or NKG2D ligation.

34. CD40-/- tumor bearing animals displayed reduced lung metastasis in comparison to tumor bearing wild type mice.

35. CD40-/- TAMs produce reduced levels of MMP-9 and VEGF in comparison to wild type TAMs

36. Tumors grown in CD40-/- mice produced significant amounts of angiogenic factors MMP-9 and VEGF, although drastically less than tumors grown in wild type mice

37. TAMs restore tumor expression of surface molecules 72

38. Tumor cytokines production induced by TAMs 


\section{INTRODUCTION}

\section{Macrophage Activation and Plasticity}

Macrophages are important components of both innate and acquired immunity. They are highly heterogeneous, and have the ability to take on many phenotypes and functional properties. Macrophages are derived from circulating monocytes and either remain motile or take residence within tissues in the host where they exhibit tissue specific morphologies and distinct functional activities crucial for homeostasis of the tissue. Many of the behaviors that macrophages display could be considered opposing in nature, for example they can participate in pro-inflammatory or anti-inflammatory activities, be tissue destructive or tissue restorative and immunogenic or tolerigenic (Fig. $1)^{1,2}$. Each of these diverse activities can be triggered by a variety of response modifiers such as cytokines, growth factors, hormones, arachidonates, stress hormones, PPARS, FABPs, etc. as well as by an activation signal received, such as TLR, CD40 and/or TNF$\mathrm{R}$ ligation ${ }^{1-5}$.

Macrophage activation traditionally refers to what is termed classical activation, or the pro-inflammatory, and phagocytic functions that macrophages employ during antimicrobial immune responses. Macrophages are among the first cells to arrive at sites of infection. Infiltrating monocytes respond to tissue cytokines and chemokines to up regulate inflammatory activities. Upon activation macrophages increase expression of -1 


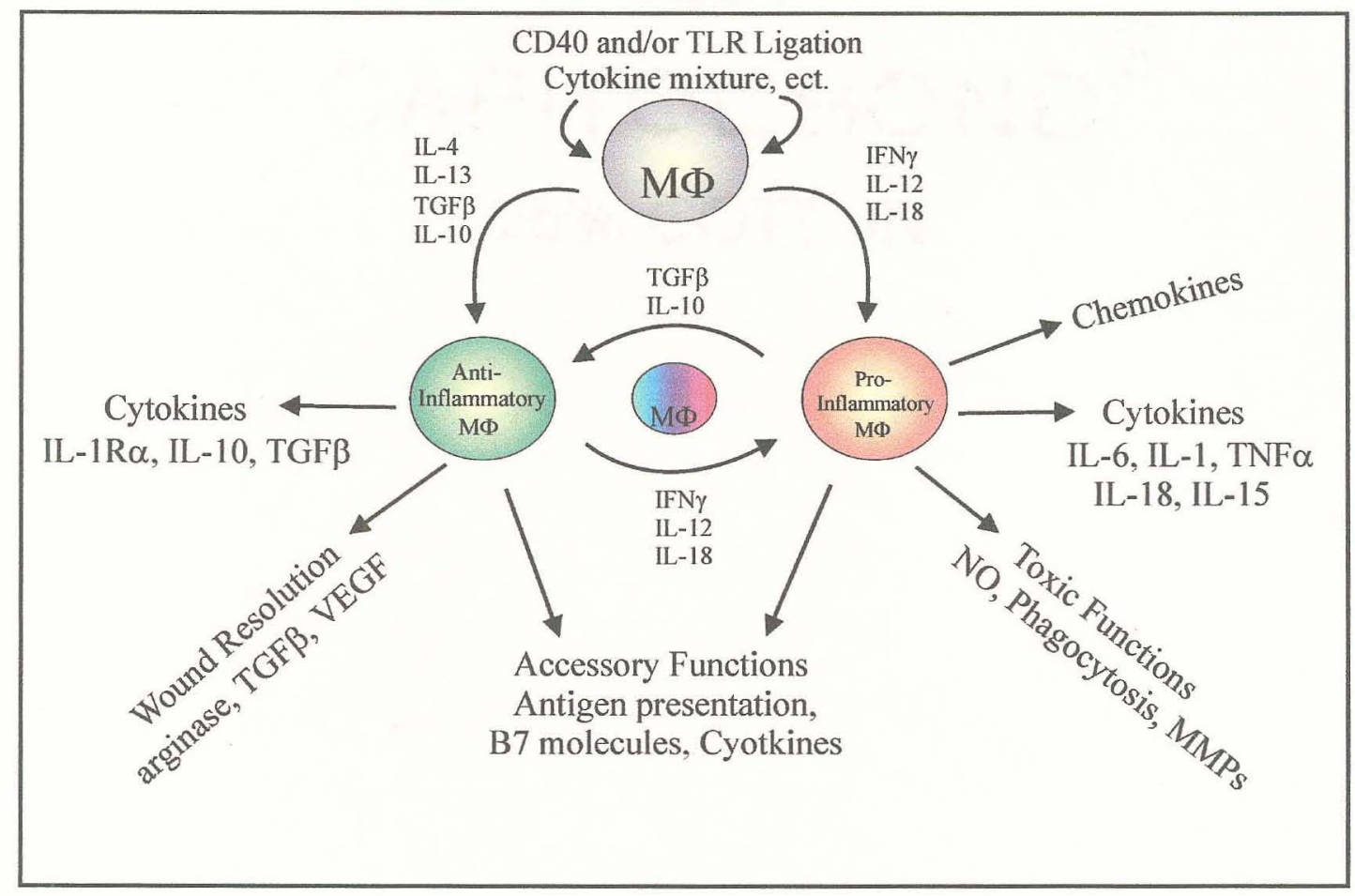

Figure 1. Macrophages are capable of many functions, many seeming opposing in nature, anti-inflammatory vs pro-inflammatory, and toxic vs healing. The function displayed is dependent upon the response modifiers encountered. Furthermore, macrophages will continue to differentially and reversibly adapt the functional profile displayed in response to progressive changes in the cytokine environment. 
CD40 and TNF receptors, elevate production of pro-inflammatory cytokines, TNF $\alpha$, IL and IL-6, and chemokines which recruit other immunological cells to the area. As the response progresses macrophages produce increased INOS and NO, participate in oxidative bursts and provide co-stimulation by the up-regulation of B7 and MHC class II molecules ${ }^{1,3}$. At this point in the response, NK and $\mathrm{T}$ cells are present and producing IFN $\gamma$. IFN $\gamma$ promotes the "generation" of the activated macrophage which is referred to as "classically activated" and displays enhanced pro-inflammatory and cytotoxic activities.

In apparent opposition to the classically activated, pro-inflammatory functions, macrophages are also among the first cells to arrive at areas of non-infectious wounding, such as a muscle tear for example. In this situation, along with tissue macrophages they promote wound healing and tissue repair by the production of growth factors, proteases and angiogenic factors ${ }^{3-5}$. Macrophages displaying wound healing or anti-inflammatory type profiles are described as being "alternatively activated." Alternative activation was first described by Siamon Gordon as macrophages activated in the presence of IL-4 or IL$13^{3}$. Others have extended this description to refer to macrophages which display any pattern of function distinctly different from classically activated macrophages, and are found to produce anti-inflammatory cytokines, such as IL-10 and TGF $\beta$, as well as having increased arginase production ${ }^{4-7}$.

Many have tried to classify macrophages into distinct populations characterized by the state of activation or functional profile observed, for example classically or alternatively activated or based on the cytokines involved in the activation, M1 for cytokines typically involved in Th1 responses and M2 for cytokines involved in Th2 
responses $^{5,7-10}$. However, it is far too simplistic to suggest that macrophages are only classically (M1) or alternatively activated (M2) or exist in a polarized state between the two functional subsets. Macrophage activation is much more complex and can not be described in a linear fashion. Efficient macrophage activation requires at least two signals. One signal being a functional modulating signal, such as a cytokine, chemokine, hormone, etc. and the second being a stimulating signal such as TLR and/or CD40 ligation or a mixture of cytokines ${ }^{1,11}$. One example of macrophage plasticity can be observed by looking at a typical response to microbial infection. Once the bacteria have been cleared, macrophages begin to phagocytose apoptotic neutrophils and down regulate production of pro-inflammatory cytokines in favor of an up-regulation of tissue restorative functions ${ }^{12-14}$. This situation provides a clear example of functional adaptivity rather than subset replacement. A second example presented by our lab demonstrates that upon activation the functional pattern displayed by macrophages depends on the nature of the stimulating ligand, the modulating stimulus encountered and the time points between when each signal was received and at what time point after activation or modulation the function was assayed '. When bone marrow derived macrophages were treated with IFN $\gamma$ for $24 \mathrm{hrs}$ prior to LPS stimulation one pattern of function was observed, showing increased TNF $\alpha, \mathrm{IL}-1 \beta$, and IL-12 production and decreased IL-10 and MCP-1 production in comparision to bone marrow derived macrophages stimulated with LPS alone. A second pattern of function that was distinctly different was then observed when the macrophages were treated with IL-4 instead of IFN $\gamma$ for $24 \mathrm{hrs}$ prior to LPS stimulation, displaying increased TNF $\alpha$, MCP-1, IL-12 and IL-6 and decreased production of IL-10. Additonally, other cytokine treatments prior to LPS 
continued to give different patterns of functions than observed from either IFN or IL-4 treatment. In demonstration that timing of stimulation is important, different patterns of function were also observed when cytokine treatment (IFN, IL-4, etc.) and LPS stimulation was administered simultaneously. Moreover, we demonstrated that the pattern of function displayed continued to adapt with progressive changes to the cytokine environment ${ }^{2,11}$. These data established the functional adaptivity hypothesis, which states that macrophages are not subsets of stably differentiated cells, but rather they are plastic in nature and respond rapidly by changing their functional profile repeatedly as the response modifiers within the environment sequentially change ${ }^{2,11}$.

\section{Tumor Associated Macrophages}

The functional adaptivity hypothesis is applicable to many disease states which affect macrophage activation and functional profiles. One of the best examples of this is macrophage function and support of cancer. Macrophages have been observed to be associated with cancer for decades. However, their role in supporting tumor development has been largely unappreciated. Only recently have the observations that macrophage densities impact clinical outcomes become respected.

Cancer progression and/or tumor development is highly dependent on the tumor cell's ability to interact with its surrounding stroma. Tumor stroma content can vary from model to model, however, most are found to contain high numbers of fibroblast, endothelial cells, and possibly most importantly, macrophages ${ }^{6}$. High densities of tumor associated macrophages (TAMs) are reported to indicate a poor prognosis, specifically in 
models of breast, prostate, ovarian, and cervical cancers ${ }^{15,16,17,18}$. Many studies have shown that macrophages are critical to tumor progression ${ }^{6,15,19-24}$. In one example, Lin et al. have shown that depletion of macrophages in breast carcinoma delayed tumor progression and reduced metastasis ${ }^{25,26}$. In another study, Nowicki et al. reported impaired tumor growth in CSF-1 deficient mice, which are macrophage deficient, as CSF-1 is required for differentiation of monocytes ${ }^{16,27}$. The roles that macrophages play in tumor development are highly dependent on the tumor micro-environment ${ }^{15,28}$.

Tumors have been shown to actively recruit macrophages by the production of an array of chemokines and growth factors, most notably MCP-1 and CSF-1 ${ }^{6,29,30}$. Tumors then seem to co-opt the normal role of macrophages to promote their development, invasion, and angiogenesis. Logically, macrophage recruitment to tumors should be immunologically beneficial to the rejection of the growing tumor. The tumor would be recognized as foreign due to tumor associated antigens (TAA) and normal macrophages would initiate an anti-tumor response, presenting TAA to T cells. However, the tumor micro environment is highly immuno-suppressive, therefore TAA are not being recognized and macrophage functions are being modulated toward pro-tumorigenic and immuno-suppressive functions by anti-inflammatory tumor produced metabolites, such as TGFß, VEGF, MMPs, and PGE2 (Fig. 2) ${ }^{11,28,31-34}$.

Of the many pro-tumorigenic functions that TAMs display, angiogenesis is one of the most important for tumor growth ${ }^{35,36}$. Tumors require angiogenesis to grow beyond a certain size. Pollard has reported TAMs are responsible for "throwing the angiogenic switch"15. TAMs contribute to angiogenesis by the production of essential factors, such as IL-1, TNF $\alpha$, IL-8, MMP-9 and VEGF. How TAMs initiate angiogenesis is poorly 


\section{Cytotoxicity}

\section{Suppression}

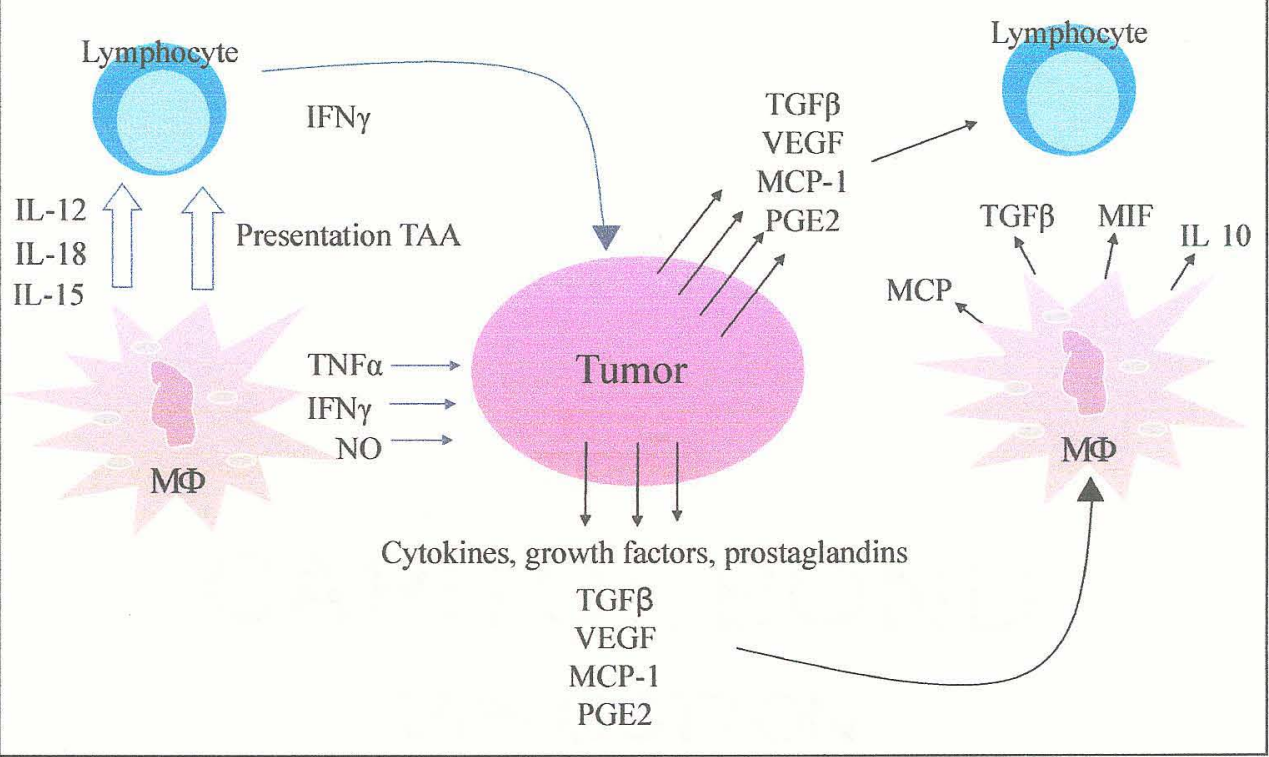

Figure 2. Macrophages which would otherwise be capable of promoting tumor destruction and cytotoxicity are influenced by metabolites of the tumor microenvironment to promote tumor growth. Tumor growth is supported by macrophage production of cytokines and growth factors, which aid in angiogenesis, tumor migration, and immunosuppression. 
understood. However, TAMs have been observed to cluster in areas of hypoxia. Hypoxia up-regulates the transcription factor, hypoxia-inducible factor $2-\alpha(\mathrm{HIF}-2 \alpha)$ in macrophages, which in turn induces VEGF expression. VEGF expression can then up regulate CSF-1, as well as act as a chemoattractant for further macrophage recruitment ${ }^{25}$, 35, 36. Other molecules influencing angiogenesis include the production of IL-1 and increased COX-2, both of which further increase HIF-1 $\alpha$, inducing transcription of VEGF ${ }^{26,37}$. MMPs, especially MMP-9 which is essential for the cleavage of biologically active VEGF, have also been shown to be up-regulated. According to reports studying tumor angiogenesis VEGF and MMPs are the most critical to vessel formation ${ }^{26,37,38}$. Production of these factors, along with cytokine production of IL-1, IL-8, and TNFo, results in the proliferation and migration of endothelial cells, matrix remodeling, and the formation of blood vessels $15,35,39,40$.

In addition to angiogenic roles, TAMs are essential in other areas of tumor development as well, including metastasis, and immunosuppression. In metastatic development, macrophages infiltrate the basement membrane. This invasion along with the production of proteases degrade the basement-membrane which creates a portal through which tumor cells can enter the stroma ${ }^{31,36}$. The tumor cells are recruited to the portal by macrophage production of growth factors which serve as chemotactic molecules allowing tumor migration and metastatic spread ${ }^{17,31}$.

In immunosuppression, several populations of "suppressor" macrophages have been described. The first set described by Bordin in 1976, and further characterized by Mantovani, referred to TAMs which are recruited from circulating blood monocytes and are believed to be mature macrophages that have been polarized by tumor products. No 
distinct phenotype has been described for this population, however they are known to produce cytokines and growth factors favorable to tumor growth such as those previously mentioned $^{5,41}$. The second population described by Bronte and Gabriolovich is referred to as an immature myeloid suppressor $(\operatorname{ImC})$ cell with a phenotype $\mathrm{CD} 11 \mathrm{~b}^{+} / \mathrm{GR} 1^{+} / \mathrm{CD} 31^{+}$. They have been described to be able to "mature" to either a type I or type II polarized functional state depending on the cytokine treatment. However, this population has only been described in the tumor in the late stages of development, typically accumulating in the spleen and other lymph tissue ${ }^{27,42-45}$. Gabriolovich reports that the ImC impair alloreactivity in vitro through a nitric oxide dependent mechanism and induces $\mathrm{T}$ cell apoptosis but require cell-cell contact ${ }^{45,46}$. The third population is described as a mature myeloid cell by Rodriguez that accounts for high arginase I production and has a $\mathrm{CD} 11 \mathrm{~b}^{+} / \mathrm{GR}-1^{-}$phenotype, but are also $\mathrm{CD} 16^{+} / \mathrm{CD} 32^{+}$and I-A/I-E ${ }^{+}$. Though the mechanisms are poorly understood, no cell-cell contact seems to be required for the inhibition of T cell activity by this population. This population of TAMs are reported to display distinct morphology consisting of irregular cytoplasms with high intracytoplasmic vacuoles and are low in phagocytic nature which makes their functional pattern unique from the other TAM populations reported ${ }^{47}$. To date there has been no relationship described for between these three individually described populations, nor has there been a study addressing, at which time point during tumor development the different functional patterns are displayed by the TAMs. TAM function is known to vary based on the locations within or around the tumor, such as within perivascular sites, stromal regions, and hypoxic or necrotic areas, as well as in the metastatic tissue ${ }^{35,36}$. Application of the functional adaptivity hypothesis may explain some of the discrepancies 
in deciphering these seemingly different TAM populations or TAMs functional profiles. The pattern of function displayed is influenced by the cytokines and growth factor components within the tissue micro environment. The levels of these metabolites change during tumor development and in different tumor micro environments (peri-vascular vs tumor parenchymal), these progressive changes would in turn cause the functional profiles of TAMs continue to shift.

\section{TAM activation}

All of the functional profiles that have been observed indicate that TAMs are actively producing cytokines, chemokines, and growth factors and contributing to tumor growth, suggesting that they are in some way activated. While it is known that tumor

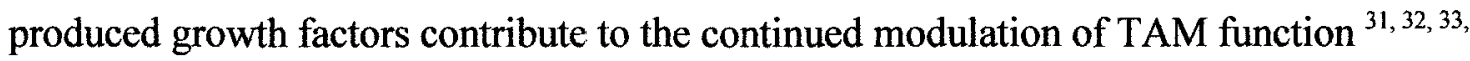
${ }^{48}$, how TAMs actually become activated to elicit pro-tumorigenic functions remains to be determined. Some speculation includes activation by recognition of tumor associated antigens (TAA) or by the mixture of cytokines present with the tumor, but these speculations do not explain the activation of TAMs residing in tissues distal to the primary tumor mass. There are reports of tumor suppression mediated by the release of membrane vesicles or exosomes. Exosomes were first described in 1987 by Johnston while studying reticulocytes from sheep ${ }^{49}$. Exosomes are formed by the inward budding of endosomal membranes, which are then referred to as multivesicular bodies, then are

then secreted from endocytic compartments after fusing with the plasma membrane ${ }^{50,51}$. Many cells have been shown to release exosomes including APCs, epithelial cells, mast 
cells, and tumor cells. Exosomes are believed to function in two ways, first they are thought to eliminate obsolete proteins during cell maturation and secondly, they have been reported to communicate among cells by cell contact, participating in immune stimulation and immune suppression ${ }^{52}$. Similarly, exosomes circulating in the tumor bearing host would interact with cells of the immune system allowing for both activation and suppression. NK cells, for example have been reported to be suppressed by interaction with the over-expression of NKG2D ligands on the surface of tumor cells and tumor released exosomes ${ }^{20,53,54}$. Further investigations of suppression reveal that tumor exosomes mediate CD8+ T cell apoptosis by Fas ligand ${ }^{55}$. Stimulation by exosomes has been evidenced by the ability of DC derived exosomes to be able to effectively activate T cells in the absence of viable APCs ${ }^{52}$. An exosome expresses the same receptors and ligands as the area of the cell membrane from which it was generated. Tumor cells express many surface molecules important in both suppression of cells of the immune system and for stimulation of tumor cell proliferation. Among the molecules reported are corresponding receptors and ligands which could theoretically allow for self stimulation such as, CD40/CD154 and NKG2D/Rae- $1^{20,53,56}$. Many other cells of the immune system also express these receptors or ligands, which provide them with activation signals. Macrophages express CD40 and be can efficiently activated by the ligation of CD40 by CD154 ${ }^{12,57}$. It has not been examined as to whether tumor exosomes interact with tissue macrophages, which may provide some insight as to how macrophages distal to the tumor mass become activated TAMs and are induced to express pro-tumorigenic, pro-metastatic and anti-inflammatory activities. 


\section{TAM Plasticity and Cytokine Therapies.}

There is evidence that TAMs are not a stable subset of pro-tumorigenic immunosuppressive cells, but rather their functional profile can change. Chu et al, have derived DCs from TAMs with IL-4 and GMCSF treatments and compared their ability to present $\mathrm{Ag}$ with DCs that were derived from peripheral blood monocytes of a healthy mouse. It was observed that DCs presenting TAA were capable of stimulating IFN $\gamma$ secretion by tumor specific T cells at the same level as, or in some cases higher levels than the peripheral blood monocyte derived DCs ${ }^{58}$. Another example generated in our lab, showed that TAMs respond to IFN $\gamma$ treatment in vitro by reducing production of protumorigenic cytokines, MCP-1 and IL-10 and increasing pro-inflammatory, TNF $\alpha$ and IL6 production in comparison to normal macrophages upon LPS stimulation ${ }^{11}$. These examples indicate that TAMs maintain functional plasticity and are capable of adapting their cytokine profiles in response to changes in environmental signals and/or stimulation. It is well established that tumor produces and inflicts its anti-inflammatory products on macrophages encouraging them to aid in tumor growth ${ }^{15}$. However, observing TAM function after interrupting the tumor environmental signals, in other words testing their adaptivity, such as through cytokine treatments has not been examined.

Cytokine therapies have previously focused on NK, NKT, and T cell responses ${ }^{13,}$ ${ }^{59-61}$. Much of the success has come from the administration of pro-inflammatory cytokines, such as IL-12 and or IL-2/IL-12. IL-12 is a heterodimeric molecule made up of an $\alpha$ chain, the $p 35$ subunit, and a $\beta$ chain, the $p 40$ subunit. The subunits are linked by a disulfide bridge, which forms the biologically active heterodimer ${ }^{14,62}$. IL-12 plays an 
essential role in interactions between the innate and adaptive responses. It is produced by macrophages, DCs, B cells, and possibly other accessory cells ${ }^{14,62}$. IL-12 activates T cell and NK cells proliferation and production of IFN $\gamma$. IL-12 was first shown to induce anti-tumor effects by Brunda et. al. ${ }^{13}$. They reported that the potent anti-tumor effects were based on the induction of IFN $\gamma$ and the activation of NK and CD8+ T cells ${ }^{13,14,63,64}$. Since then there have been many studies further supporting IL-12 anti-tumor activity in various tumor models, including melanomas, colon cancers, and mammary carcinomas ${ }^{60}$, 65. In addition to effector cell activation IL-12 has been shown to possess potent antiangiogenic capabilities and can inhibit growth factor production. Even in light of the many beneficial anti-tumor effects of IL-12, there have been severe difficulties with clinical trials due to considerable toxicity when administered systemically. Hill and Egilmez, as well as many others have developed methods of administration to overcome clinical toxicity by encapsulating IL-12 in lipid microspheres or nanovessicals, alone or with other cytokines such as IL-2 and GMCSF in the treatment regime ${ }^{23,61,66,67}$. This method allows for both local delivery as well as for a slow release of the cytokines, enabling the initiation of an effective response, while minimizing side effects and toxicity ${ }^{61,66}$. Hill et al. showed significant results in that IL-12 and GMCSF delivered in microspheres greatly reduced tumor proliferation, metastasis, and recurrence, and ultimately enhancing survival by $60 \%{ }^{61}$. The study showed that the anti-tumor effect was IFN $\gamma$ mediated and required a NK and NKT cell response as well as a long term, tumor specific adaptive $\mathrm{T}$ cell response ${ }^{23,61,66}$. Trinchieri et al. confirmed the findings of Hill, and showed that the effectiveness of IL-12 therapy is IFN $\gamma$ mediated through the induction of CXC chemokines, such as IP-10 and $\mathrm{MIG}^{60,68}$. 
Other pro-inflammatory cytokines, such as IL-18 and most impressively IL-15 have been reported to act synergistically with IL-12, further enhancing anti-tumor responses ${ }^{69,70}$. IL-15 was identified by its structural and physiological similarities to IL2 and by its ability to stimulate proliferation of the IL-2 dependent CTLL-2 T cell line in the presence of neutralizing Abs to IL-2 ${ }^{2,71}$. Much is still unknown of the synthesis and release of IL-15, however, it is known to activate through one or a combination of its receptors, IL-15R $\alpha, \beta$,or $\gamma$ in soluble or, most often, a membrane bound form ${ }^{71,72}$. IL-15 is produced by many cell types including skeletal muscle, BM stromal cells, thymic epithelia, heart, lung, and monocytes and macrophages. Monocytes and macrophages were the first cell types to be reported as a functionally relevant source of IL-15 in the context of the immune response ${ }^{71}$. IL-15 has an essential role in the development and activation of many immune cells ${ }^{73-75}$. IL-15 is required for NK cell development as is demonstrated by the absence of NK cells in mice deficient in IL-15, IL-15R, and/or in the JAK3 and STAT5 signaling pathways, which are required for IL-15 production or signaling by IL-15R ${ }^{76,77}$. Furthermore, IL-15 is essential for NK proliferation, cytotoxicity, and cytokine production, as well as for NK/macrophage interactions ${ }^{71,72,78}$. In addition to NK cells IL-15 is also essential to chemotaxis, proliferation, production of cytokines, protection from apoptosis and for cytotoxicity in T cells ${ }^{79-81}$. Again, mice deficient in IL-15 or IL-15R lack functional CD8 + T cells and have minimal reactive CD4+ T cells ${ }^{75,81,82}$. In the context of cancer, it was recently discovered that IL-15 could rescue tumor suppressed CD8+ T cells, allowing for enhanced survival ${ }^{79,81,83,84}$. This revelation may be a key in understanding the mechanisms by which cytokine therapies may be improved to be more effective. 


\section{Hypothesis, Significance, and Summary.}

To date, all studies regarding cytokine therapies have focused on the adaptive anti-tumor response generated 6 or more days after treatment ${ }^{61,85,86}$. However, the initial events from hours to several days following treatment have been ignored. The early events of cytokine therapy are likely to induce the production and/or release of other cytokines, such as IL-18 and/or IL-15 which would act synergistically with the administered cytokine in promoting the later observed adaptive response. It is unlikely that the potent adaptive anti-tumor response could take place without first reducing the highly immuno-suppressive influence of the tumor environment. In realization of this some studies have tried to circumvent the suppressive tumor environment by surgically removing the primary tumor mass prior to administering cytokine treatment ${ }^{61}$. While the effects of removing the primary tumor mass would be beneficial, we hypothesize that the disruption of the apparent tumor, macrophage relationship may provide the needed window of opportunity for the activation and promotion of the adaptive anti-tumor response. Although, macrophages are not activated by IL-12, they are responsive in so far as their functional profile is modulated by IL-12. Given the elevated production of pro-tumorigenic cytokines and growth factors observed to be produced in the tumor bearing host, we furthermore hypothesize that TAMs are activated in a tumor-supportive fashion in situ, but will adapt their function to a more proimmunogenic profile in response to IL-12 treatment (Fig.3).

In this study, macrophage activation by NK interactions, as well as by soluble tumor produced molecules, tumor cells and tumor exosome interactions were examined, 
before and after IL-12 treatment. Macrophages were observed to be activated by cell-cell contact with tumor cells and/or tumor exosomes, which was prevented by the blockade of either CD40 or NKG2D ligation. Activated TAMs rapidly responded to IL-12 treatment by increasing pro-inflammatory cytokine production and releasing their intracellular store of IL-15. The release of IL-15 was observed to mediate infiltration of CD4+ and CD8+ T cells into the tumor mass and metastatic lung. Furthermore, IL-15 release and lymphocyte infiltration coincided with tumor destruction and metastatic clearance. The observations made in this study provide insight as to the tumor/TAM relationship and on the impact of IL-12 treatment in the adaptation of the TAM functional profile in vitro and in vivo, which may contribute to the promotion of the anti-tumor response and ultimately destruction of the tumor and/or metastases. 


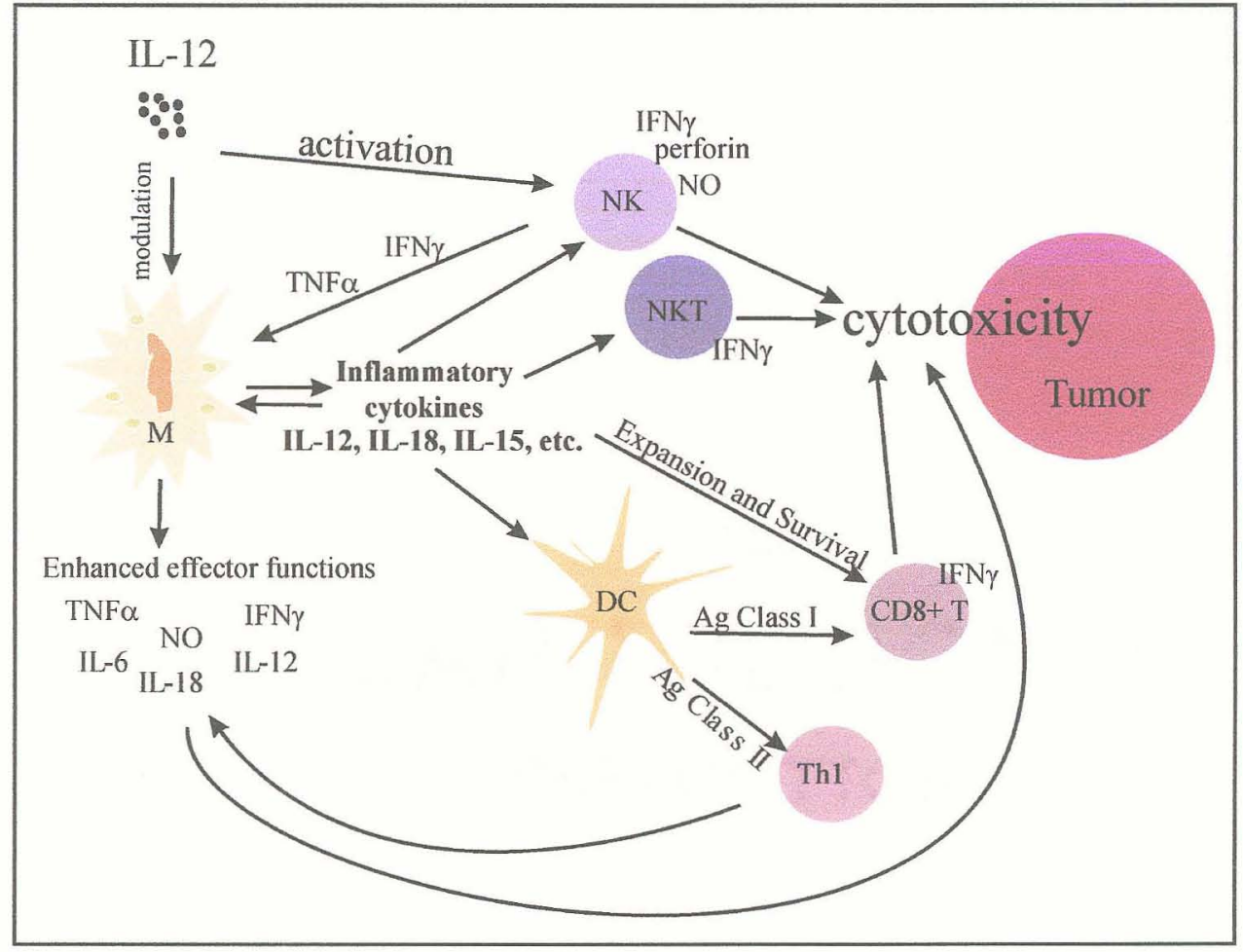

Figure 3. TAMs are modulated by IL-12 treatment to increase inflammatory cytokine production and release of IL-12, IL-18, and IL-15. The inflammatory cytokines then work synergistically with the IL-12 treatment to rescue and activate tumor suppressed NK, NKT cells and to promote the expansion and survival of CD8+ T cells. The motivated cells and enhanced effector functions promote cytotoxicity and tumor destruction. 


\title{
MATERIALS AND METHODS
}

\begin{abstract}
Animals. C57Bl/6J, C57Bl/6J -CD40 $0^{\mathrm{tm} / \mathrm{Kik} / \mathrm{J}}, \mathrm{C} 57 \mathrm{Bl} / 6 \mathrm{~J}-\mathrm{Lyst}^{\mathrm{tg}-\mathrm{T}}$, and DBA/2J female mice were obtained from Jackson Laboratories. C57BL/6J-IL-15KO mice were obtained from Taconic Farms. All animal protocols received prior approval of the Institutional Animal Care and Use Committee and all experiments were performed in accordance with relevant guidelines and regulations.
\end{abstract}

Tumor models. Lewis Lung carcinoma (3LLC) transfected to express membrane human Muc-1was obtained from Dr. Gordon Ross, Brown Cancer Center, Louisville, KY. TC-1, B16F1, and LL-2 tumor lines were purchased from ATCC. Tumors were maintained by in vivo passage with limited intermittent culture in vitro. Tumor cells were injected s.c. in the left side of $\mathrm{C} 57 \mathrm{BL} / 6 \mathrm{~J}$ mice. Tumors were measured at 3 day intervals with calipers at two bisecting diameters and an approximate volume is calculated by the formula (0.4) $\mathrm{x}$ (large diameter) $\mathrm{x}$ (small diameter) ${ }^{2}$. Tumors were excised at $0.4-0.6 \mathrm{cc}$, diced and passed through a sieve (Bellco, Vineland NJ). The cells were washed with Dulbecco's PBS + 2\% FBS and cultured for 2-5 3 day cycles in RPMI1640 with 5\% FBS, HEPES, and gentamycin before re-passage in vivo. Tumors with necrosis or with volumes twofold higher or lower than the mean of the group of mice were not used as a source of tissue for that experiment. 
Macrophages. Macrophages were purified from single cell suspensions of lung, spleen, peritoneal lavage, and tumor mass of tumor bearing animals by magnetic bead separation using first a negative selection with anti-CD19 and anti-CD5 and then positive selection with anti-CD11b (Mac-1) (Miltenyi Biotech, Auburn CA). The lungs and tumors were excised after perfusion with PBS-EDTA and removal of the mediastinal lymph nodes. Samples were treated with collagenase and DNase (Sigma) in $37^{\circ}$ water bath for $30 \mathrm{~min}$. Dispersed cells were then centrifuged over a discontinuous $30 / 35 / 45 / 70 \%$ percoll gradient. Macrophages were purified by magnetic bead separation from the cells banding at the $45 / 70 \%$ interface. Purity of $>95 \% \mathrm{CD} 1 \mathrm{~b}^{+}$/ F480 was confirmed by flow cytometry for all 4 macrophage populations. Macrophages were cultured in RPMI1640 supplemented with $5 \%$ fetal calf serum, HEPES, and gentamycin and were activated by addition of $100 \mathrm{ng} / \mathrm{ml}$ LPS where indicated.

NK cell isolation. NK cells were purified from single cell suspensions of the spleen from normal animals by magnetic bead selection using the DX5 magnetic beads and columns from Miltenyi Biotech.

Exosomes. Tumor exosomes were purified by magnetic bead selection. Tumor supernatants from cultures of adherent tumor cells were collected and centrifuged at 1500 rpm for $10 \mathrm{~min}$. to remove any detached intact tumor cells. $4 \mu \mathrm{g}$ of biotinylated antiBCP8 (MUC-1) was added to $5 \mathrm{ml}$ of tumor supernatant for 15 minutes at room temperature. Anti-biotin magnetic beads (Miltenyi Biotech) were then added to the 
supernatant for an additional 15 minutes at room temperature. Exosomes were then positively selected for using Macs columns (Miltenyi Biotech, Auburn CA).

In vitro Cytokine treatment. Macrophages were plated at $1 \times 10^{5} / \mathrm{ml}$ in a 96 well plate for cytokine analysis or a 6 well plate for flow cytometric analysis. rm-IFN $\gamma$ was added to cultures at $20 \mathrm{u} / \mathrm{ml}$ and soluble $\mathrm{rm}-\mathrm{IL}-12$ was added to cultures at $5 \mathrm{ng} / \mathrm{ml}$. Cultures were incubated from $17-48 \mathrm{hrs}$ as indicated.

In vivo IL-12 treatment. Interleukin-12 containing microspheres were prepared by phase inversion nanoencapsulation and supplied by Dr. Nejat Egilmez, State University, Buffalo NY ${ }^{61,66,67}$. Microspheres were re-suspended at $1 \mathrm{mg} / 100 \mu 1$ in PBS for injection into the center of the tumor mass.

Flow Cytometry. Cells were treated at $1 \mu \mathrm{g} / 10^{6}$ cells with Fc block (Pharmingen) for 15 minutes in the presence of $0.1 \%$ azide and $3 \%$ heat-inactivated fetal bovine serum (staining buffer). The cells were then incubated with fluorochrome conjugated antibodies at appropriate dilutions in staining buffer for 30 minutes on ice, washed and analyzed on a B-D FacsCalibur. Typically 10,000-20,000 cells were analyzed for their expression of surface proteins. Antibodies reactive with MAC-1, CD40, CD154, CD44, H2Kb, Gr-1, F480, CD69, CD45, CD8, CD4, CD3, IL-15R $\beta$, and NK1.1 were purchased from BD Pharmingen, anti-CD314 (NKG2D/CX5) was purchased from Biolegend, anti-hMuc-1 (BCP8) provided by Jun Yan, Brown Cancer Center, Louisville, KY, and pan-specific anti-Rae-1 was purchased from R\&D Systems. Intracellular IL-15 was assayed using the 
BD Pharmingen cytofix/cytoperm kit per manufacturer's instruction along with the biotinylated goat anti-mouse IgG IL-15 antibody (R\&D Systems) with a secondary streptavadin Ab (BD Pharmingen). Un-labeled anti-IL-15 (Novus Biochemicals) was used as an $\mathrm{Ab}$ control for intracellular staining. Appropriate isotype controls were ran for each $\mathrm{Ab}$ used.

SuperArrray. For the GEArray cells were purified and lysed in Trizol for a cholorform extraction. Briefly $200 \mu \mathrm{l}$ of choloroform was added to cell lysates and centrifuged. The aqueous phase was collected and an equal amount of (25:24:1) phenol : choloroform : isoamyl alcohol was added and centrifuged. The aqueous phase was collected and washed with isopropanol, centrifuged and was with ice-cold $75 \%$ ethanol. After centrifugation the pellet was air dried, then resuspended in nuclease-free water for further purification by Qiagen Mini Elute RNA easy clean-up kit according to the manufacturer's instructions. The GEArray was then run according to manufacturer's instructions using $3 \mu \mathrm{g}$ purified RNA. Data was analyzed by software provided by SuperArray for the GEArray Q series kit (software available on SuperArray website www. Superarray.com).

Quantitative Real Time PCR. cDNA was produced from mRNA from $10^{6}$ cells from independent cell culture experiments (in treated and untreated tumor bearing and normal mice) by using $\mu \mathrm{MACS}^{\mathrm{TM}}$ One-Step cDNA kit (Miltenyi Biotec). The cDNA was used for quantitative real-time polymerase chain reaction (PCR) amplification with Sybr Green using gene specific primers for MIF (Superarray), TGFß, IL-15, and IL-18 (Maxim), and Klrk-1 (NKG2D) (Qiagen). Duplicate cDNA template samples were amplified and 
analyzed in the MJ Opticon thermal cycler with conditions of $96^{\circ} \mathrm{C}$ for $15 \mathrm{~min}$ followed by 40 cycles of $95^{\circ} \mathrm{C}$ for $30 \mathrm{sec}, 58^{\circ} \mathrm{C}$ for $30 \mathrm{~s}$ and $30 \mathrm{~s}$ at 72 with a plate read for gene expression between each cycle. A standard curve of cycle thresholds using 10 fold serial dilutions of cDNA samples was established and used to calculate the relative abundance of the target gene of each cytokine and vehicle control samples. Samples were run according to manufactures protocol and analyzed as fold increases or decreases between normal and tumor bearing animals after normalization to a $\beta$-actin control. Differences between groups were analyzed using the REST program.

Cytokine Analysis. Cytokines were assayed using Becton-Dickinson's cytokine bead array kit and a BD FacsCalibur according to manufacturer's instructions. Sample supernatants were analyzed undiluted and also at a 1:50 dilution. ELISAs for TGFb, VEGF, and MMP-9 were performed using the Quantikine kits from R\&D Systems, as per manufacture's instructions. The IL-15 ELISA was performed using rat anti-mouse IgG2a IL-15 $\mathrm{Ab}$ (R\&D Systems) at $4 \mathrm{mg} / \mathrm{ml}$ as the capture $\mathrm{Ab}$ in combination with the biotinylated goat anti-mouse IgG IL-15 antibody (R\&D Systems) at $400 \mathrm{ng} / \mathrm{ml}$ as the detection $\mathrm{Ab}$.

Blocking Abs for in vivo studies. Neutralizing anti-IL-15 and IgG isotype control was obtained from eBioscience. Anti-IL-15 or the isotype control was injected i.p. At $500 \mathrm{ng}$ $1 \mathrm{hr}$ prior to intra-tumor IL-12 microsphere treatment. Anti-NK1.1 was obtained from BD Pharmingen and injected at $250 \mathrm{ng} 24$ hrs prior to IL-12 microsphere treatment. 
Blocking Abs for in vitro studies. Anti-NKG2D (R\&D Systems) was added to macrophages in vitro at $2 \mu \mathrm{g} / \mathrm{ml}, 5 \mu \mathrm{g} / \mathrm{ml}$, or $10 \mu \mathrm{g} / \mathrm{ml}$ to block NKG2D ligation. To measure specificity of the blocking anti-NKG2D, Fc-rmNKG2D purchased from R\&D systems was added to cultures at $1,2,5$, or $10 \mu \mathrm{m} / \mathrm{ml}$. Anti-CD154 (Biolegend) was added to $3 \mathrm{LLC}$ cells and exosomes with and without macrophages in vitro at $5 \mu \mathrm{g} / \mathrm{ml}$, $10 \mu \mathrm{g} / \mathrm{ml}$, or $25 \mu \mathrm{g} / \mathrm{ml}$ to block the tumor cell ligation of macrophage CD40.

Infiltration Studies. Mice were injected with 3LLC tumor cells subcutaneously, when the tumor measured $0.5 \mathrm{cc}$ anti-IL-15 or anti-NK1.1 were injected $1 \mathrm{hr}$ and $24 \mathrm{hrs}$ prior to IL-12 microsphere injection respectively. Three and 5 days post treatment tissues were harvested. Mice were purfused with $10 \mathrm{ml}$ cold PBS, and the tumor mass and lungs were removed. Tumor diameter was recorded as previously mentioned. Wet weights of the tumor were recorded and digital images were obtained. The tissues were then pressed between frosted slides to acquire single cell suspensions. Cell populations were then stained for flow cytometric analysis.

Histology. Animals bearing tumors were purfused with $10 \mathrm{ml}$ PBS prior to lung extraction. The lungs were then harvested and kept in individual cassettes which were placed in $10 \%$ buffered formalin for 16 hours, washed in $70 \% \mathrm{EtOH}$ and taken to University of Louisville School of Medicine, Pathology Department, (Louisville KY) for sectioning and H\&E staining. 
Digital Images. Digital images of the slides obtained from pathology services were taken with a Nikon coolpix digital camera at 10x magnification on a microscope. Slides were also scanned on a HP scanner unmagnified to count visible metastatic nodes.

Statistical analysis. For flow cytometric analysis of the neutralization of IL-15 or depletion of NK cell studies, the mean and standard deviation was calculated on the number of infiltrating CD4+/CD45+ and CD8+/CD45+ cells per $1 \times 10^{6}$ tumor cells or $1 \times 10^{6}$ viable lung cells recorded from 5 mice or 3 mice respectively. The significance between groups ( 5 individual mice/group) for cytokine production assayed by ELISA, CBA, or qrt-PCR, and tumor volumes and masses were determined by the two sample $t$ test. 


\section{RESULTS}

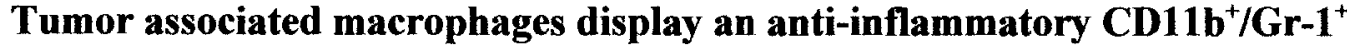 phenotype that shifts in response to inflammatory cytokine treatment.}

An accumulation of myeloid or macrophage suppressor cells have been observed in many tissues of tumor bearing hosts. The cells are characterized by a CD1 $1 b^{+} / \mathrm{Gr}-1^{+}$ phenotype and are thought to be contribute significantly to the suppression of the immune response by the production of anti-inflammatory or pro-tumorigenic cytokines. Primarily these cells have been reported to originate from the bone marrow and infiltrate tissues such as the spleen, liver, and lung and aid in metastatic development as well as immune suppression ${ }^{19,35,43,87}$. To determine whether resident macrophages from the peritoneum of tumor burdened animals displayed the anti-inflammatory, "suppressor" phenotype, macrophages were purified from tumor-bearing or healthy mice. The cells were then stained with anti-CD11b and anti-Gr-1 (LY6G/C) fluorescent antibodies for flow cytometry analysis. The surface phenotype of macrophages from the tumor bearing animals display a shift from $\mathrm{CD} 11 \mathrm{~b}^{\mathrm{Hi}}$ to $\mathrm{CD} 11 \mathrm{~b}^{\mathrm{Low}}$ in comparison to normal peritoneal macrophages. Concurrent with the shift in $\mathrm{CD} 11 \mathrm{~b}$ expression, approximately half of the CD1 $1 b^{+}$TAM population also expressed the Gr- $1^{+}$phenotype (Fig 4.). Similarly TAMs purified from the spleen and lung of tumor mice also displayed an increase in Gr-1 


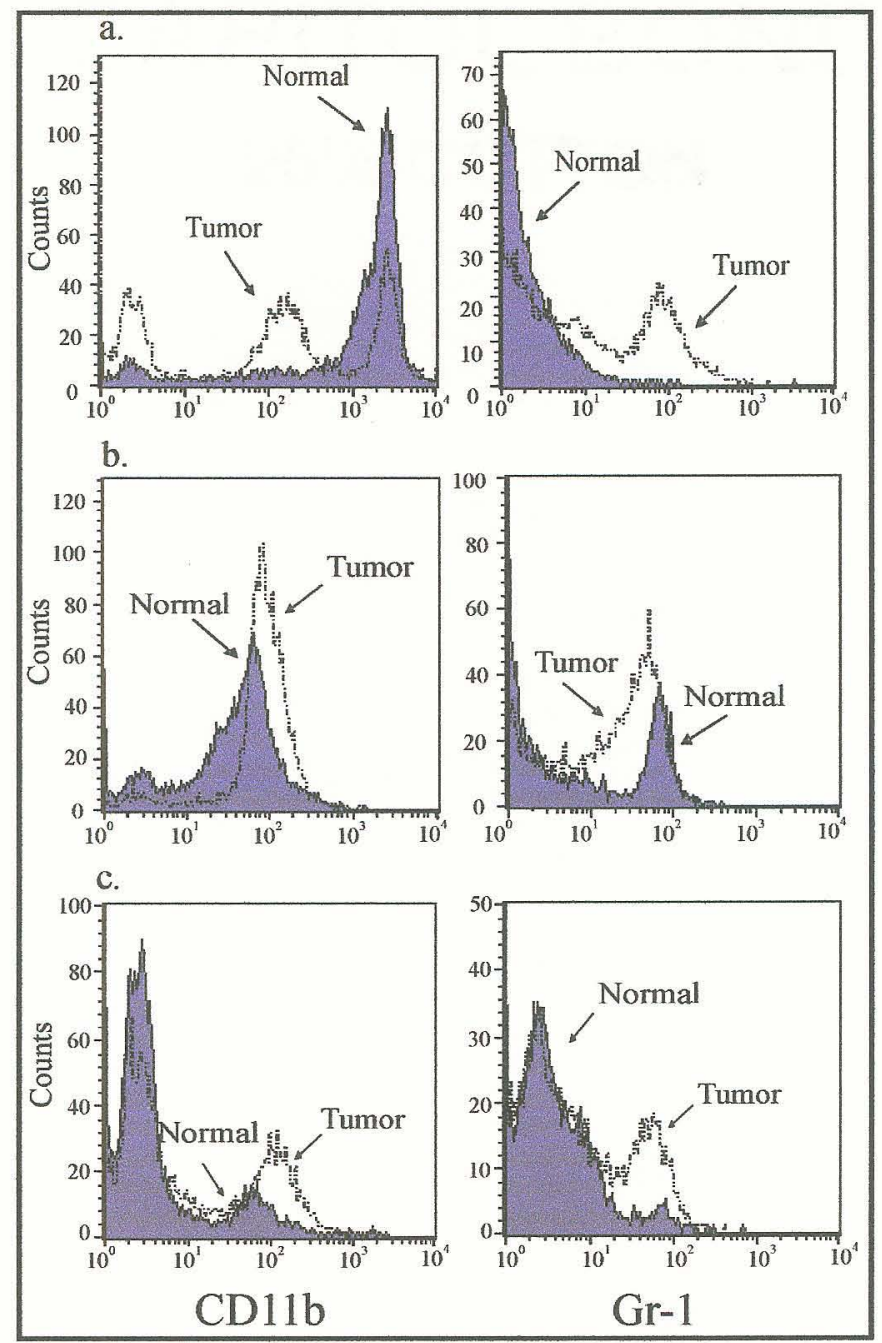

Figure 4. Tumor associated macrophages display the anti-inflammatory "suppressor" phenotype similar to tumor infiltrating macrophages. Macrophages from tumor bearing and normal mice were enriched from the a) peritoneum, b) spleen, and c) lungs and stained for cell surface expression of CD11b and Gr-1 (Ly6G/C). Results are representative of three independent trials. 
expression. Macrophages however, are known to have transient phenotypes and change functions due to changes in the micro-environment ${ }^{1,2,11}$. Therefore to determine if the phenotype of these macrophages was stable, TAMs were treated in vitro with proinflammatory cytokine IFN $\gamma$ (Fig. 5) for 24 hours. Subequent flow cytometric analysis revealed that the Gr- $1^{+}$expression receded and the $\mathrm{CD} 11 \mathrm{~b}^{\mathrm{Hi}}$ phenotype was restored (Fig. 5)

In addition to their distinctive surface phenotype it has been reported and we have confirmed that, TAMs produce elevated levels of pro-tumorigenic cytokines, such as MCP-1 and TGF $\beta$ in response to LPS stimulation in comparison to normal macrophages from the peritoneum. (Fig.6). Treatment overnight with IFN $\gamma$ prior to LPS stimulation reduced pro-tumorigenic cytokines production (Fig.6)

\section{IL-12 treatment in vitro alters the functional profile of TIMs and TAMs.}

While IFN $\gamma$ treatment overnight is effective for altering macrophage function in vitro, pro-inflammatory cytokines, such as IL-12 have been shown to induce an antitumor response with less toxicity when delivered locally to the tumor ${ }^{61,66,67}$. To gain a broader perspective of the differences in cytokine gene expression between normal macrophages and TAMs and to define the impact of IL-12 treatment, macrophages were isolated from the peritoneal cavity of normal and tumor bearing mice and cultured overnight with or without IL-12. SuperArray analysis indicated that many cytokine genes were down-regulated in TAMs, most notably IFN $\gamma$ and TNF $\alpha$, as well as several cytokine 


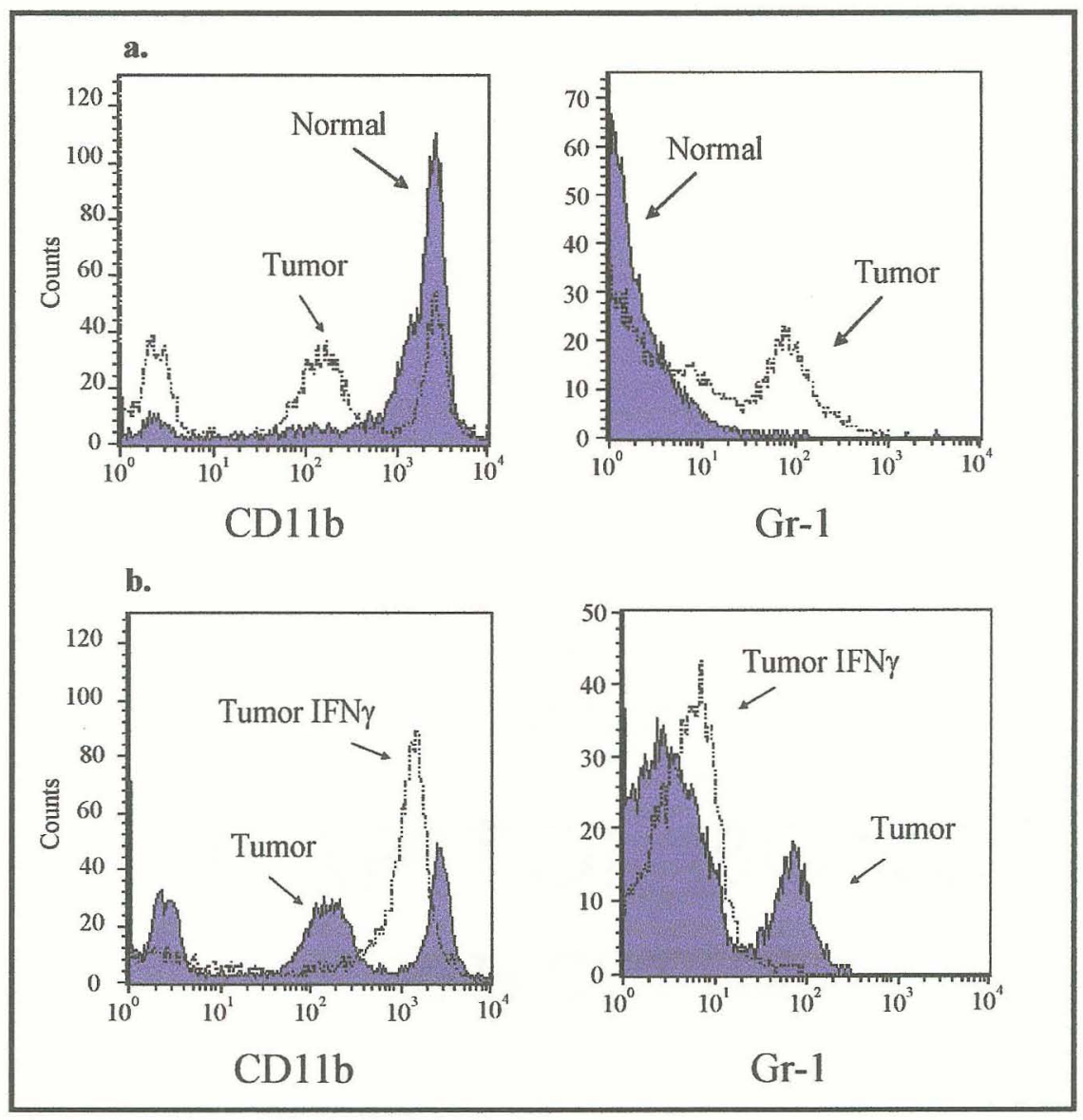

Figure 5. IFN $\gamma$ treatment restores $\mathrm{CD} 11 \mathrm{~b}^{\text {hi }}$, Gr- $1^{-}$phenotype. Macrophage were purified from peritoneal lavage of normal or tumor bearing mice and stained for cell surface expression of CD11b and Gr-1 before and after overnight culture with $20 \mathrm{u} / \mathrm{ml}$ IFN $\gamma$. Results are representative of 4 independent trials. 


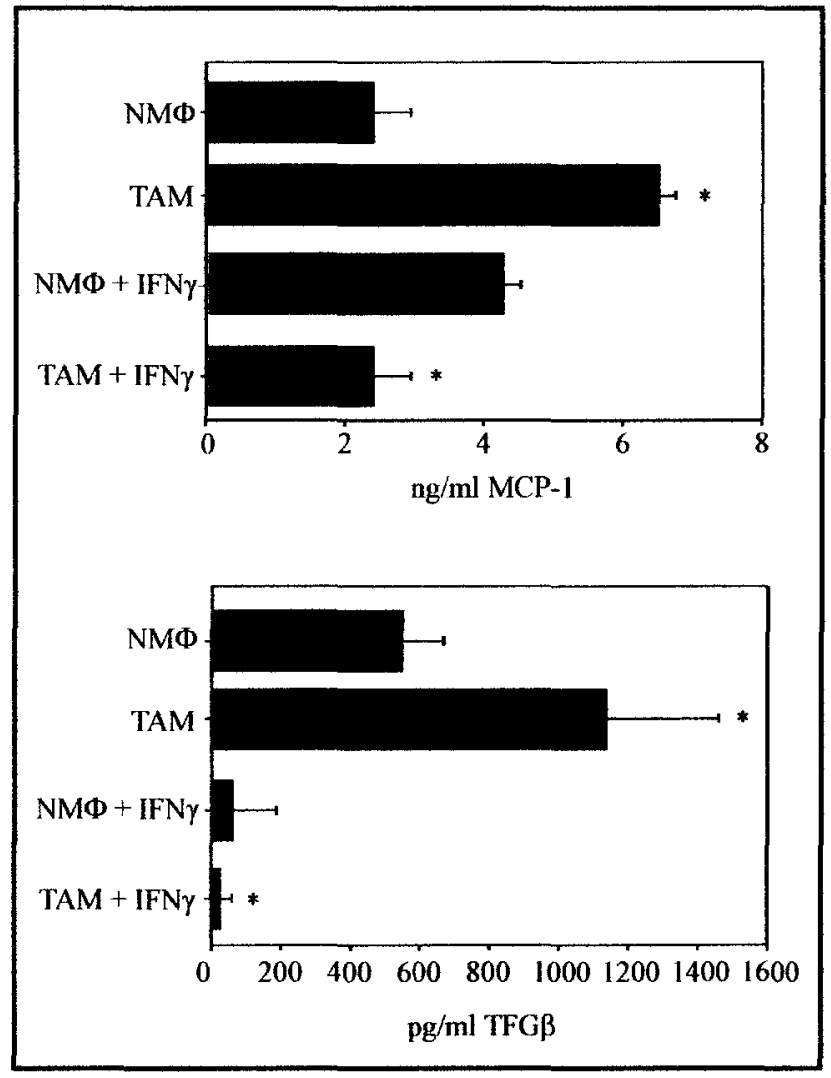

Figure 6. IFN $\gamma$ reduces TAM production of pro-tumorigenic cytokines in response to LPS stimulation. MФs were purified from normal or tumor bearing mice and immediately or after overnight culture with $20 \mathrm{u} / \mathrm{ml}$ IFN $\gamma$ stimulated with LPS. Supernatants were then harvested and analyzed for cytokine production by ELISA. Data expressed as mean \pm s.d. of triplicate cultures. ${ }^{*} p<0.05$, comparing groups normal vs TAM and TAM vs TAM + IFN $\gamma$. Data are representative of 3 independent trials. 
receptor and chemokine genes. Other genes such as, MIF, IL-10 and CCR2, which are products known to aid in tumor development were greatly up-regulated in TAMs compared to normal macrophages (Fig. 7a). Overnight treatment with IL-12 appeared to enhance expression of the genes that were down-regulated in TAMs and simultaneously reduced the expression of the genes that were up-regulated in TAMs (Fig. 7b). Based on the results of this assay two pro-tumorigenic cytokines genes of interest, TGF $\beta$ and MIF, that were found to be significantly up-regulated by the presence of the tumor and a proinflammatory cytokine gene of interest, IL-15 that were found to be up-regulated by IL12 treatment of TAMs by the superarray (Fig. 7b) were selected for quantitative analysis by real time qrt-PCR. TAMs expressed a greater than 9-fold increase in expression of TGF $\beta$ and MIF mRNA but very little IL-15 or IL-18 mRNA compared to normal peritoneal macrophages (Fig.8). Overnight culture with IL-12 reduced the expression of both TGF $\beta$ and MIF genes to near background levels and induced a 3-fold increase in expression of IL-18 mRNA and a 20-fold increase in expression of IL-15 mRNA (Fig.8).

Given the vast differences in gene expression we then analyzed the pattern of cytokine production elicited by activation in vitro, the tumor infiltrating (TIMs) and peritoneal, splenic or lung TAMs were stimulated with $100 \mathrm{ng} / \mathrm{ml}$ LPS immediately ex vivo or after overnight culture with rIL-12. IL-12 treatment reduced or abrogated MCP-1 production and elevated TNF $\alpha$ and IL- 6 production by TIMs as well as by peritoneal, splenic and lung TAMs (Fig.9). The IL-12 treatment reduced IL-10 production by splenic and lung TAMs (Fig. 9) but elevated IL-10 production by peritoneal TAMs (Fig.9). 


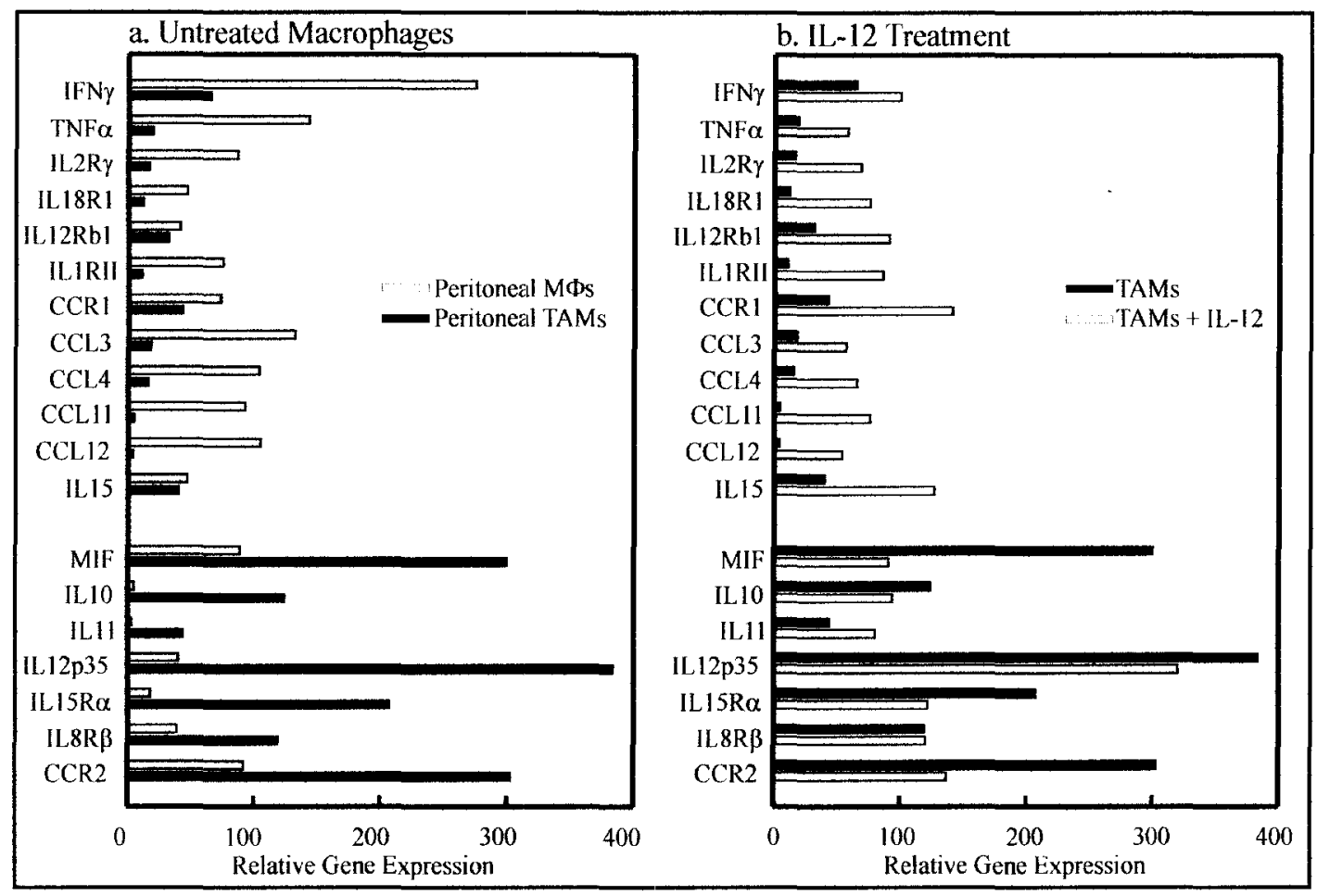

Figure 7. IL-12 impact on cytokine gene expression of TAMs. Peritoneal M $\Phi$ from normal or tumor-bearing mice (a) or from tumor bearing mice treated with placebo or IL-12 microspheres (b) were analyzed by Superarray for expression of cytokine cytokine receptor or chemokine mRNA. Results shown are from a single gene array, however gene expressions were confirmed by multiple qrt-PCRs. 


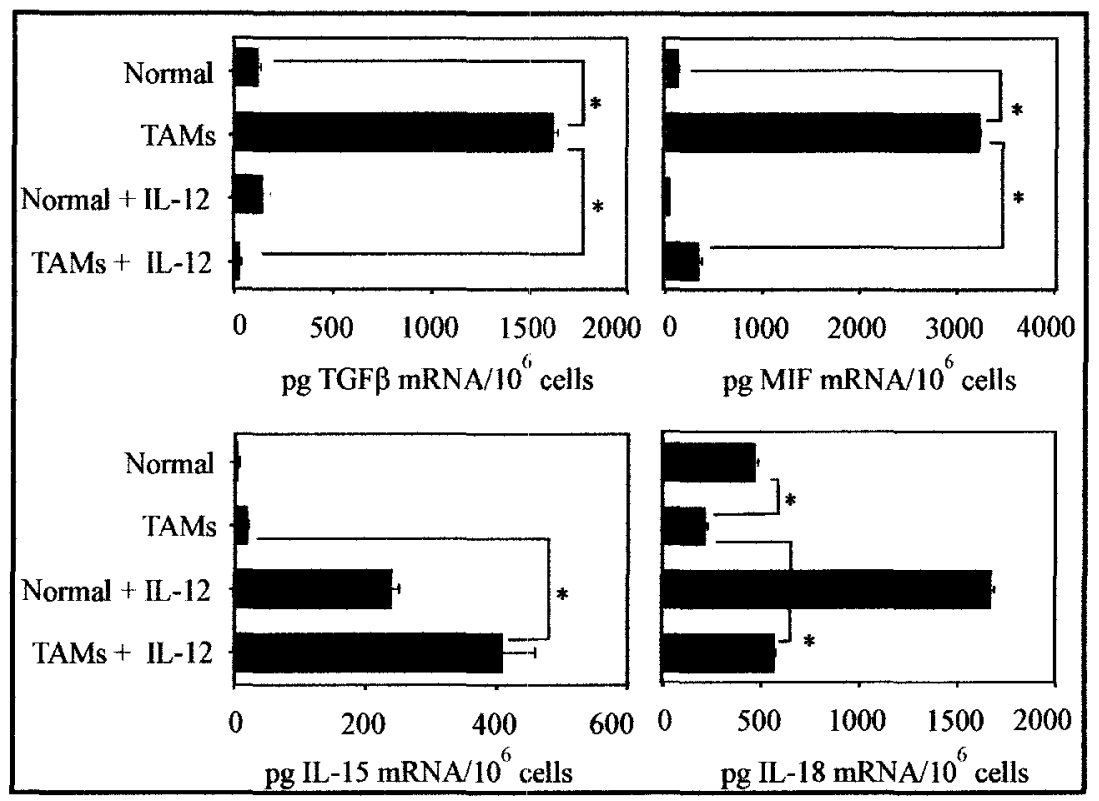

Figure 8. In vitro treatment alters the cytokine gene expression pattern. Macrophages were purified from peritoneal lavage of tumor bearing and normal mice and cultured overnight in the presence or absence of $5 \mathrm{ng} / \mathrm{ml} \mathrm{IL-12.} \mathrm{RNA} \mathrm{was} \mathrm{extracted} \mathrm{and} \mathrm{analyzed}$ for expression of TGF $\beta$, MIF, IL-15 and IL-18 by qrt-PCR. Data are expressed as 1 pg mRNA $/ 10^{6}$ cells \pm s.d. of 3 mice, ${ }^{*} p<0.001$ between normal macrophages and TAM or between TAM and TAM + IL-12. Data represent 4 independent trials. 
Therefore, for each tissue analyzed, IL-12 treatment altered the pattern of the cytokine response to LPS stimulation.

\section{IL-12 treatment in vivo alters the functional profile of TIMs and TAMs.}

IL-12 effectively modified macrophage cytokine production in vitro, but to determine if IL-12 could alter the function of TAMs in vivo, mice bearing subcutaneous 3LLC tumors were treated with IL-12 encapsulated microspheres. Peritoneal macrophages were harvested from 90 min to 5 days post injection and assayed for cytokine production following LPS stimulation. IL-12 treatment induced a dramatic shift in cytokine production in the peritoneal TAMs as early as 90 minutes after treatment (Fig. 10). The elevated production of MCP-1 and IL-10 elicited from peritoneal TAMs from the placebo-treated tumor-bearing mice was reduced 2-3 fold in TAMs from the IL-12 treated mice. Furthermore the production of TNF $\alpha$ was observed to be elevated in TAMs following IL-12 treatment. This shift in functional profile was sustained for at least 5 days after IL-12 treatment (Fig. 10). Purified lung and splenic TAMS (Fig. 11a) displayed the same shift in their functional profile as peritoneal TAMs after injection of the IL-12 microspheres. TAMs from spleen and lung displayed elevated production of MCP-1 and IL-10 and decreased production of IL-6 relative to normal controls. Three hours after IL12 treatment, the splenic and lung TAMs displayed a 3-fold or greater reduction in MCP1 and IL-10 production in response to LPS stimulation in vitro and a comparable increase in TNF $\alpha$ and IL-6 production (Fig. 11a). Purified TIMs from IL-12 treated mice displayed 


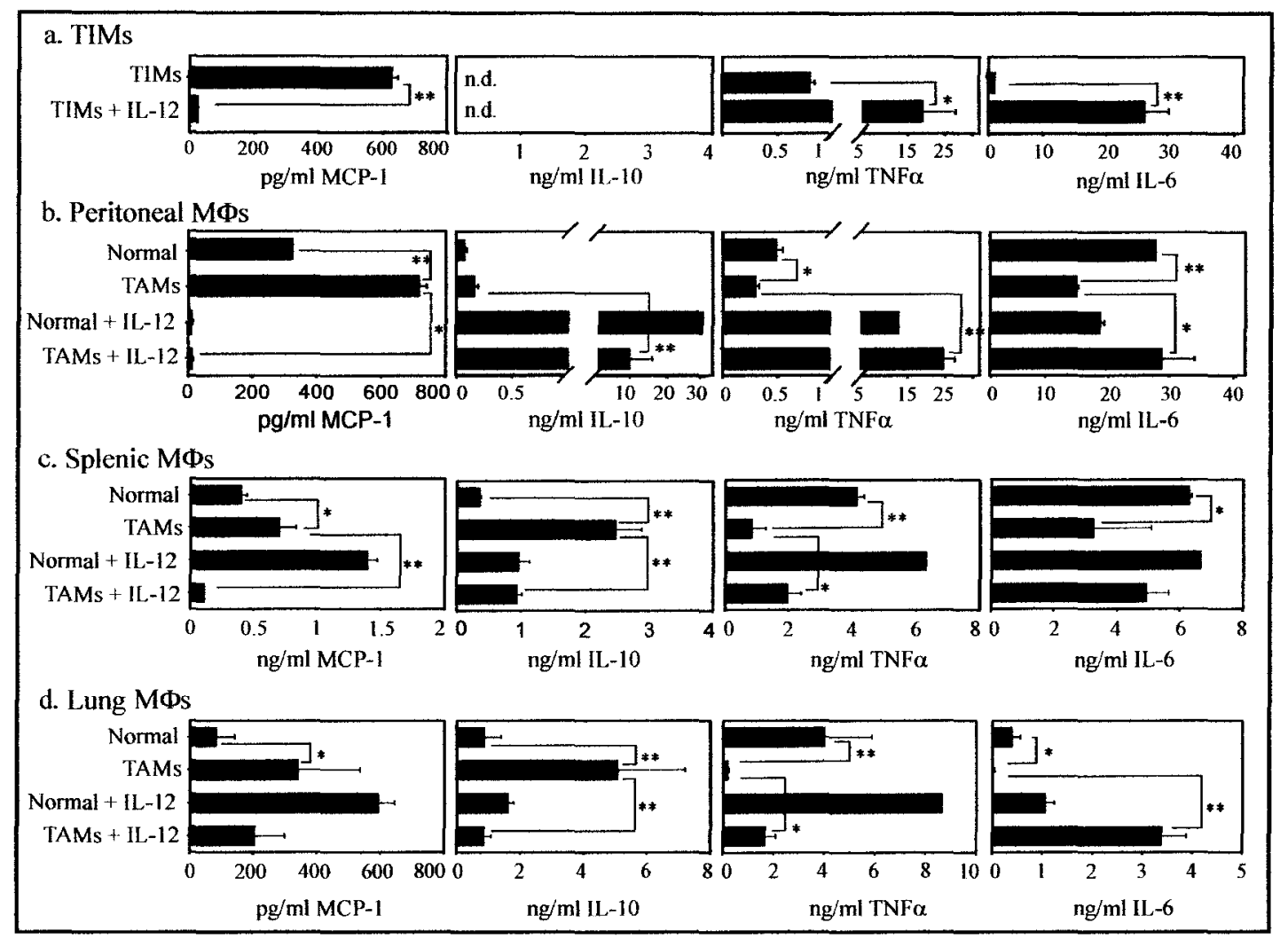

Figure 9. IL-12 changes the pattern of cytokine production by TIMs and TAMs in vitro in response to LPS stimulation. Macrophages were purified from a) the tumor, b) peritoneal lavage, c) spleen, and d) lung tissue of normal or tumor bearing mice. The cell cultures were stimulated with $100 \mathrm{ng} / \mathrm{ml}$ LPS immediately or after overnight culture with $5 \mathrm{ng} / \mathrm{ml}$ IL-12. The culture supernatants were collected and analyzed for cytokines by CBA. Data are displayed as mean \pm s.d. of macrophages from 3 mice, ${ }^{*} p<0.05,{ }^{* *} p<0.01$. Groups compare normal vs TAM and TAM vs TAM + IL-12. n.d. = none detected. The results are representative of four independent trials. 


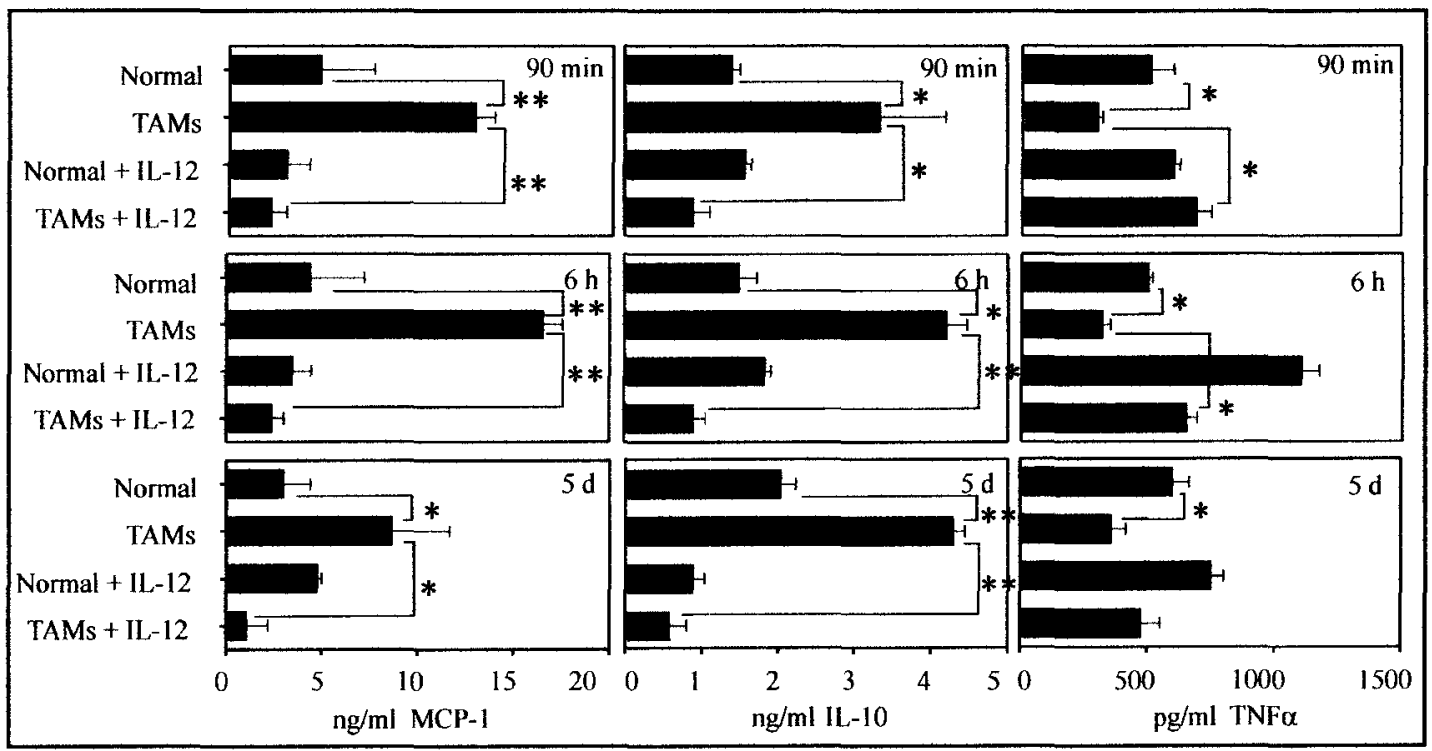

Figure 10. In vivo IL-12 treatment induces a rapid shift in the cytokine profile that is sustained for at least 5 days. Peritoneal MФs were purified $90 \mathrm{~min}$ to $12 \mathrm{hrs}$ after injection of IL-12 microspheres subcutaneously (normal mice) or into the tumor mass. The MФs were stimulated with $100 \mathrm{ng} / \mathrm{ml} \mathrm{LPS} \mathrm{and} \mathrm{the} \mathrm{supernatant} \mathrm{collected} 17 \mathrm{hrs}$ later for cytokine analysis. Data is displayed as mean + s.d. of 3 mice. ${ }^{*} \mathrm{p}<0.05 .{ }^{* *} \mathrm{p}<0.01$. Results are representative of 4 independent trials. 


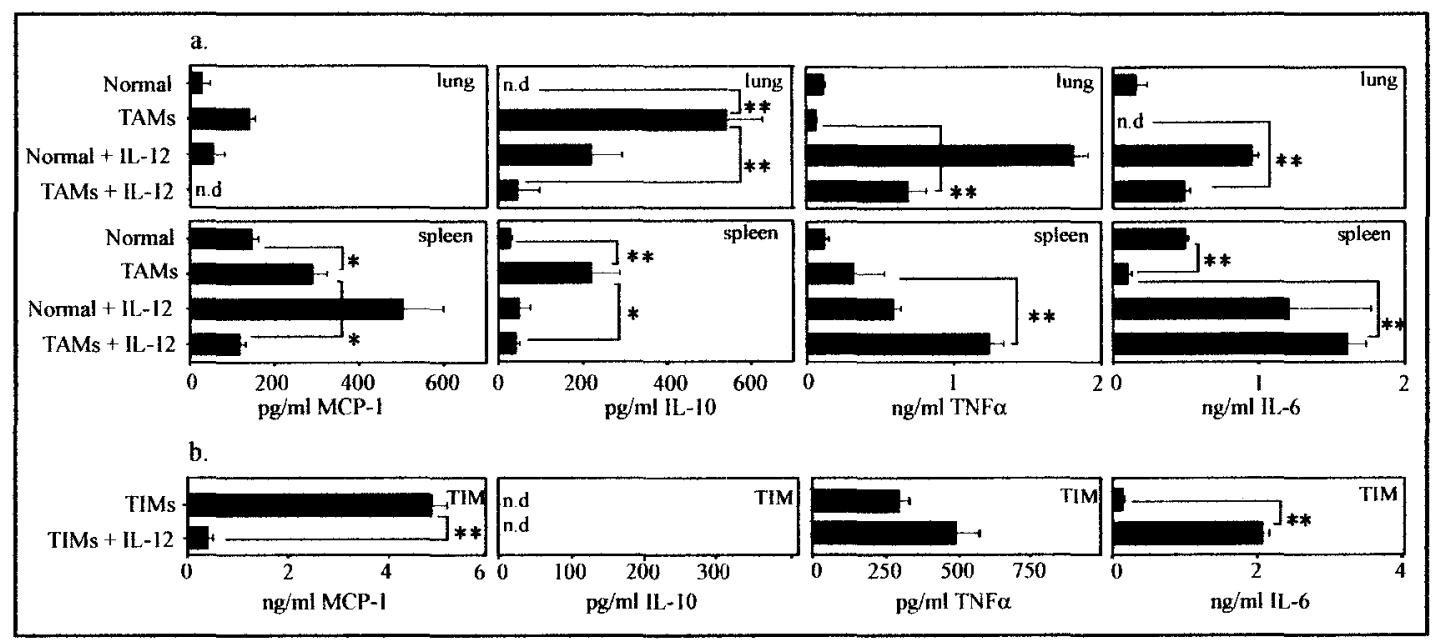

Figure 11. In vivo injection of IL-12 induces a rapid shift to pro-inflammatory functions systemically. a) Lung and splenic or b) tumor infiltrating MФs were purified $3 \mathrm{hrs}$ after IL-12 injection. The cells were stimulated with LPS and the supernatant collected $17 \mathrm{hrs}$ later for cytokine analysis. Data is displayed as mean \pm s.d. of 3 mice per group.

${ }^{*}$ indicates $\mathrm{p}<0.05$. ${ }^{* *}$ indicates $\mathrm{p}<0.01$. n.d. $=$ none detected. Results are representative of 4 independent trials. 
enhanced IL-6 production and dramatically decreased MCP-1 production as compared to placebo (Fig. 11b). Unlike TAMs, TIMs consistently failed to produce IL-10 in response to LPS stimulation in these studies (Fig. 11b). We attributed the differences observed in cytokine production by TIMs and TAMs to differences in the tissue environments from which they were purified.

\section{IL-12 induces release of $I L-15$ in tumor bearing mice.}

Analysis of TAMs from the spleen, lung, and peritoneal cavity ex vivo for cytokine gene expression corroborated previous reports that genes for MIF and TGF $\beta$ were significantly elevated (Fig.12a) ${ }^{5,33,36,88,89}$. Within 90 min after IL-12 treatment in vivo, the splenic, lung, and peritoneal TAMs displayed almost a complete abrogation of expression of MIF and TGF $\beta$ mRNA. This sharp down-regulation of tumor-supportive TGF $\beta$ and MIF gene expression was accompanied by a greater than 10 fold increase in expression of IL-15 and IL-18 mRNA relative to placebo (Fig.12b ). Therefore, the same impact of IL-12 on expression of these 4 cytokine genes by TAMs was observed both in vitro (Fig. 8) and in vivo (Fig. 12a,b). IL-15 administration to tumor bearing mice has been reported to rescue anergic/tolerant tumor-specific cytotoxic $\mathrm{T}$ cells . The dramatic IL-12 mediated up regulation of IL-15 gene expression (Fig. 12b ) was therefore of particular interest. To determine if IL-15 protein was being released as a result of IL-12 treatment, a blood sample was obtained from mice 2-24 $\mathrm{h}$ after IL-12 treatment. Analysis revealed that $3 \mathrm{~h}$ after IL-12 treatment there was a rapid appearance of IL-15 in the serum of tumor-bearing mice, attaining $>6$-fold higher concentration than that observed in 


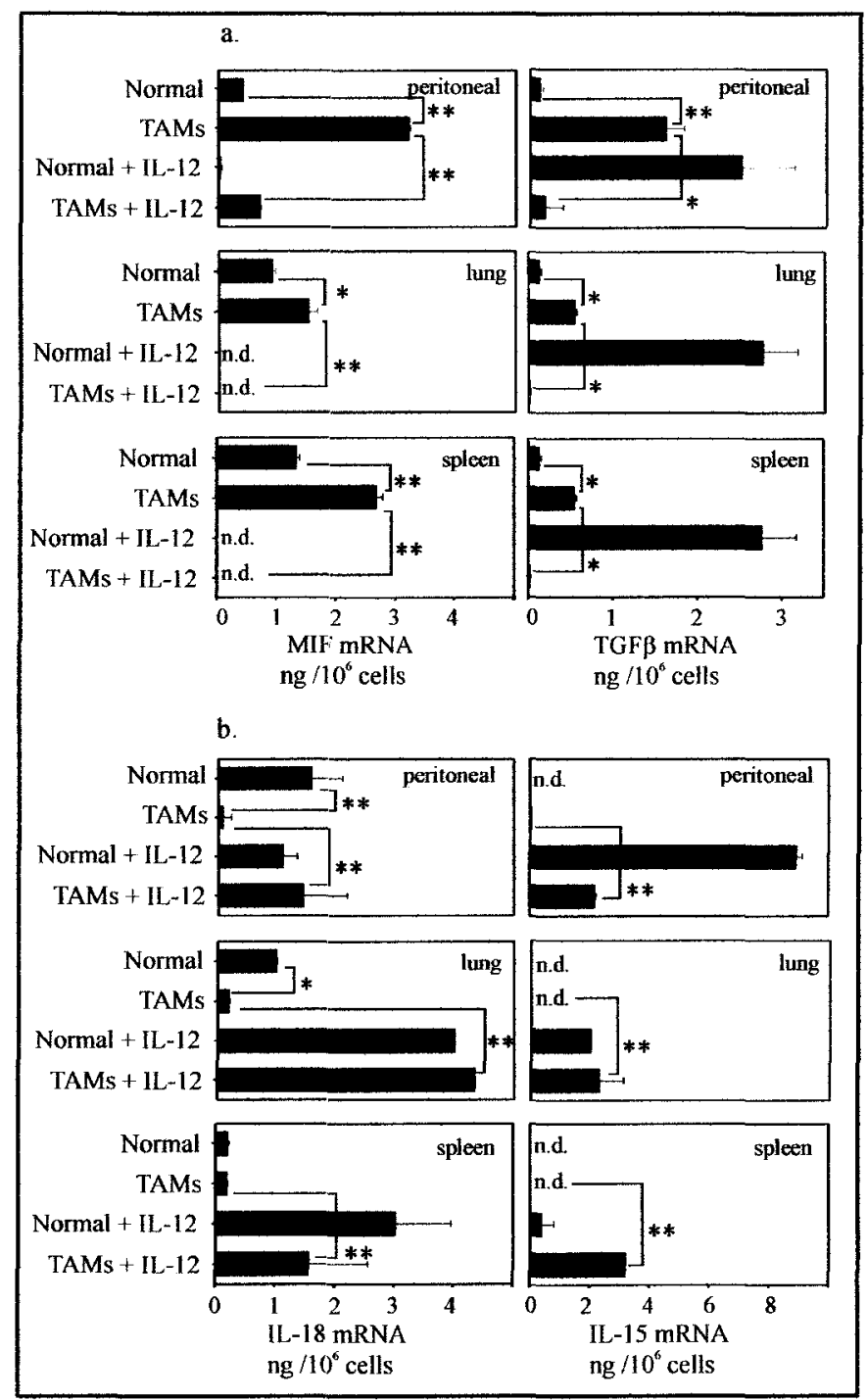

Figure 12. IL-12 injection induces a decrease in pro-tumorigenic and an increase in proimmunogenic cytokine genes. Peritoneal, lung, and splenic macrophages were purified from normal or tumor bearing mice 90 min after IL-12 injection. The cells were immediately lysed for mRNA isolation for qrt-PCR to assay a) pro-tumorigenic MIF and $\mathrm{TGFb}$ and $\mathrm{b}$ ) pro-immunogenic genes $\mathrm{IL}-18$ and $\mathrm{IL}-15$. Data are expressed as ng of mRNA $/ 10^{6}$ cells \pm s.d. of 3 mice, ${ }^{*} p<0.05,{ }^{* *} p<0.01$. N.d. $=$ none detected. Groups compare normal macrophages vs TAMs and TAMs + placebo treatment vs TAMs + IL12 treatment. 
normal mice treated with IL-12 (Fig. 13a). The contribution of TAMs to this IL-15 release was investigated by collecting peritoneal macrophages before and after IL-12 treatment and analyzing for the level of cytoplasmic IL-15. IL-12 treatment of normal mice induced an increase in cytoplasmic IL-15 in a small fraction of the macrophages whereas $\mathrm{IL}-12$ treatment of tumor bearing mice resulted in nearly a complete depletion of cytoplasmic IL-15 (Fig. 13b,c). IL-15 appeared to be released rapidly as a bolus reaching maximum concentration in the serum around 3-4 hrs after IL-12 injection which corresponded to a complete depletion of cytoplasmic IL-15 (Fig.13b,c). Serum IL-15 also disappeared rapidly, as the cytokine was undetectable in the serum $24 \mathrm{hrs}$ after IL-12 treatment. Macrophage intracellular IL-15 which was decreased at 2-3 hours after IL-12 treatment was vastly increased by 18-24 hours after IL-12 injection (Fig 14).

\section{Activated macrophages from tumor animals aid in the initiation of immuno- activation in response to IL-12 treatment.}

To determine if the IL-12 induced release of intracellular IL-15 played a role in IL-12 initiation of a tumor destructive response, anti-IL-15 or an IgG isotype control was injected into tumor bearing mice $1 \mathrm{hr}$ prior to intra-tumor injection of IL-12. Lymphocyte infiltration into the tumor and metastatic lung tissue were then measured 3 and 5 days later by flow cytometry, and the size and integrity of the primary tumor mass and extent of lung metastasis were assessed 10 days post IL-12 injection. Five days after IL-12 injection there was a 10 fold increase in infiltrating CD4+ and CD8 $+\mathrm{T}$ cells within the primary tumor. This increase was not observed in mice treated with 


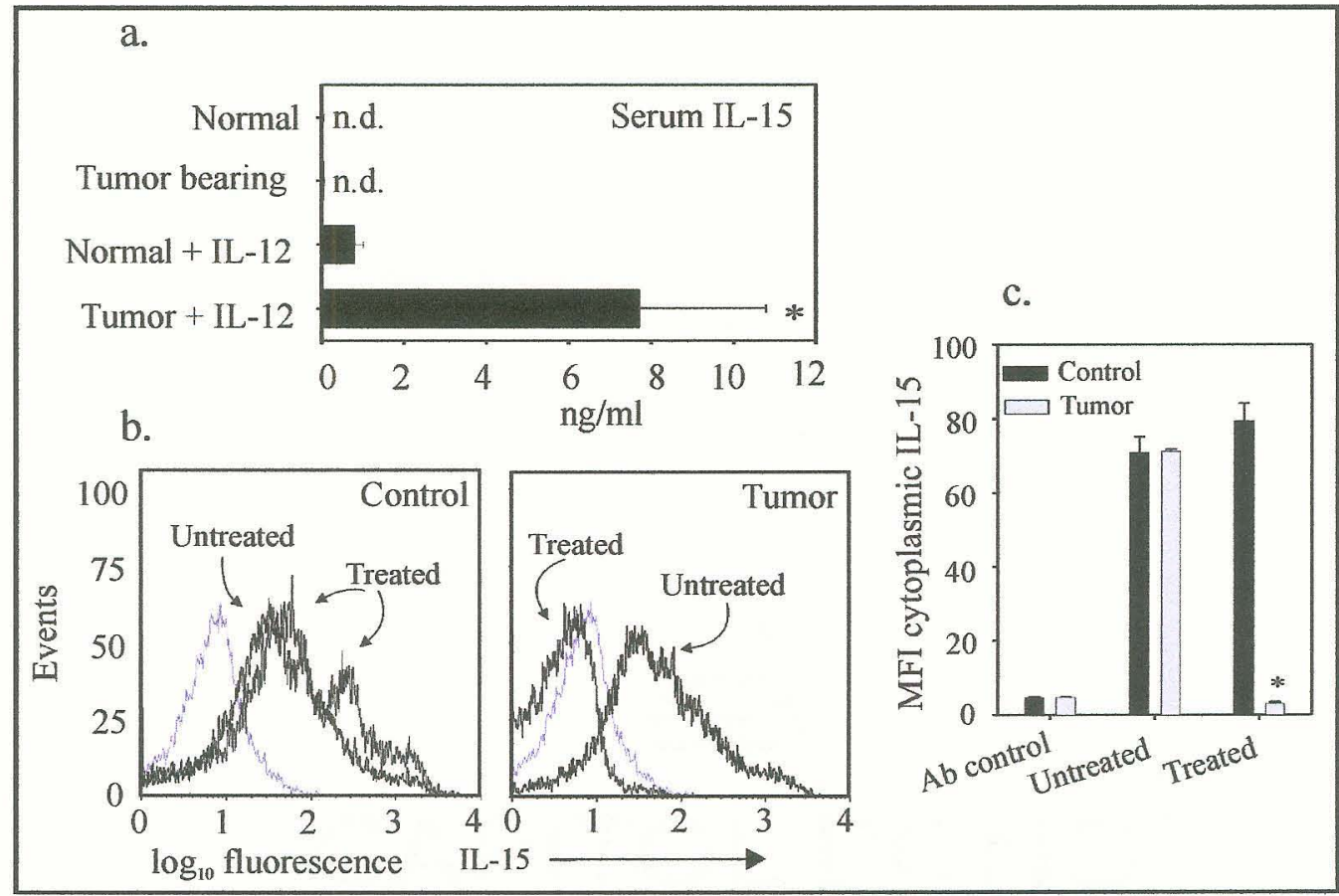

Figure 13. TAMs release IL-15 in vivo in response to IL-12 treatment. a) A blood sample was collected from normal or tumor bearing mice $3 \mathrm{hrs}$ post IL-12 injection. Serum IL-15 was assayed by ELISA. Data is displayed as mean \pm s.d. of 3 mice. $p=0.012$ for normal versus tumor-bearing IL- 12 treated mice. $n . d .=$ none detected. Results are representative of 2 independent trials (b) Peritoneal MФs from normal (left panel) or tumor bearing (right panel) mice were analyzed for cytoplasmic IL-15 3 hrs after IL-12 injection. Specificity was controlled by pre-labeling with cold anti-IL-15. c) Mean fluorescence intensity displayed a significant shift in the TAM IL-12 treated group compared to the normal MФ treated group. Data are displayed as mean \pm s.d. of 3 mice per group. Results are representative of 2 independent trials. 


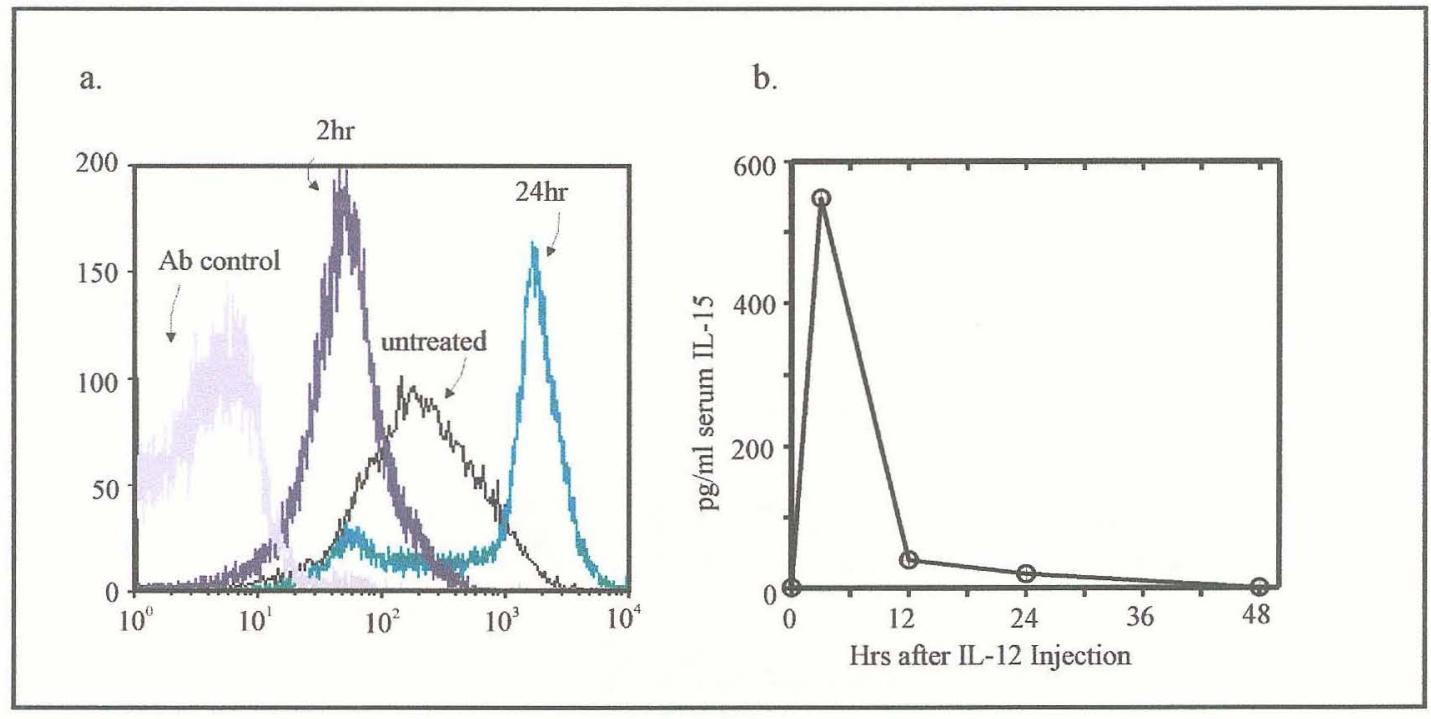

Figure 14. TAM cytoplasmic IL-15 is released rapidly as a bolus within 2-3 hrs and is replenished by 24 hrs after IL-12 treatment. a) Macrophages from tumor bearing mice were purified from peritoneal lavage before or 2-24 hrs after IL-12 injection and stained for intracellular IL-15. Specificity was controlled by pre-labeling with cold anti-IL-15. b) A blood sample was taken to measure serum levels of IL-15 by ELISA at time points 0 , $3,12,24$, and 48 hrs after IL-12 injection. Data is representative of 2 independent trials. 
anti-IL-15 + IL-12 (Fig. 15). By 10 days post IL-12 injection the tumor volume and tumor mass were significantly reduced in the IL-12 treated and IL-12 + isotype control treated groups in comparison to the untreated control group (Fig. 16a). In the IL-12 treated and IL-12 + isotype control treated groups the tumor masses were visibly ulcerated (Fig. 16b) whereas there was no indication of ulceration in the untreated control. The volume and mass of the subcutaneous tumor harvested from mice treated with anti-IL-15 + IL-12 were similar to untreated control group, the anti-IL-15 + IL-12 group did not display visible signs of ulceration (Fig.16b). Furthermore, an H\&E stain of cross sections of the primary tumor mass show both infiltration of lymphocytes and as well as the breakdown of the tumor stroma in the IL-12 and IL-12 + isotype control groups, but not in the untreated control or the anti-IL15 + IL-12 treated groups(Fig. 16c). Similarly, metastatic lungs harvested from mice treated with IL-12 displayed a significant infiltration of CD4+ and CD8 $+\mathrm{T}$ cells and contained dramatically reduced frequency and size of metastases (Fig. 17). The lungs harvested from mice treated with anti-IL-15 + IL12 displayed very little lymphocytic infiltration and displayed increased frequency and size of metastatic lesions compared to the group treated with IL-12 alone (Fig. 18). However, the lungs of anti-IL-15 treated mice did not display as extensive metastatic tumor growth as the untreated control mice. When these experiments examining IL-12 induced tumor and metastasis destruction were carried out for longer period of time, mice receiving IL-12 treatment had an $80 \%$ survival rate up to 30 days post IL-12 treatment at which point the untreated control group or animals lacking IL-15, IL-15-/- mice or where release of IL-15 was neutralized in wild type C57BL/6 mice, had expired. 


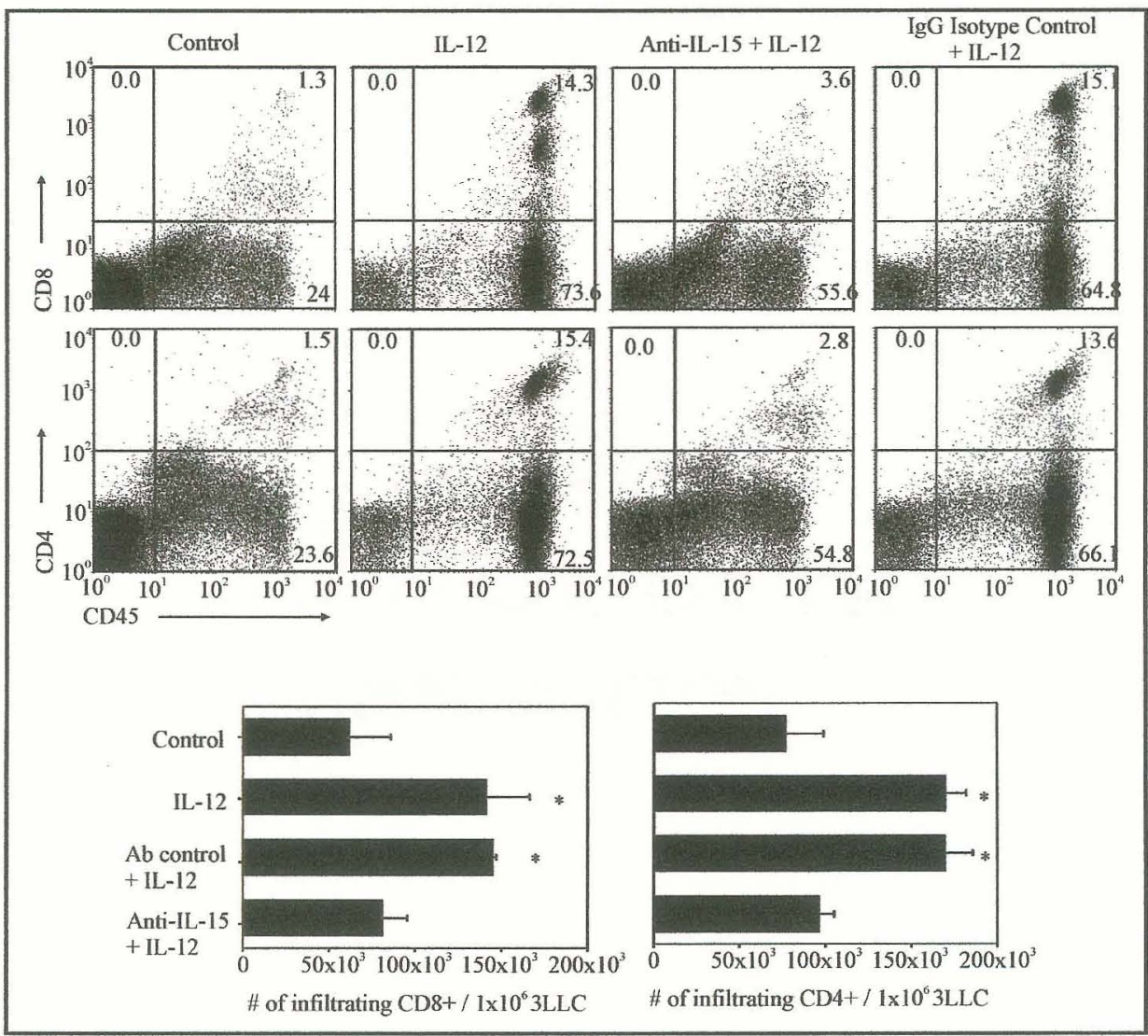

Figure 15. Neutralization of IL-15 reduces T cell infiltration in response to IL-12. Tumor bearing mice were injected with placebo or IL-12 encapsulated microspheres. One group was injected with $250 \mathrm{ng}$ anti-IL-15, $1 \mathrm{hr}$ prior to $\mathrm{IL}-12$ treatment. The tumor mass was then harvested and analyzed for infiltrating cells 3 and 5 days after IL-12 injection. Data are expressed as the mean \pm s.d. of 5 mice per group, $* p<0.05$ comparing control group or anti-IL15 group to IL-12 or IL-12 + isotype control treated groups. Results represent 2 independent trials. 


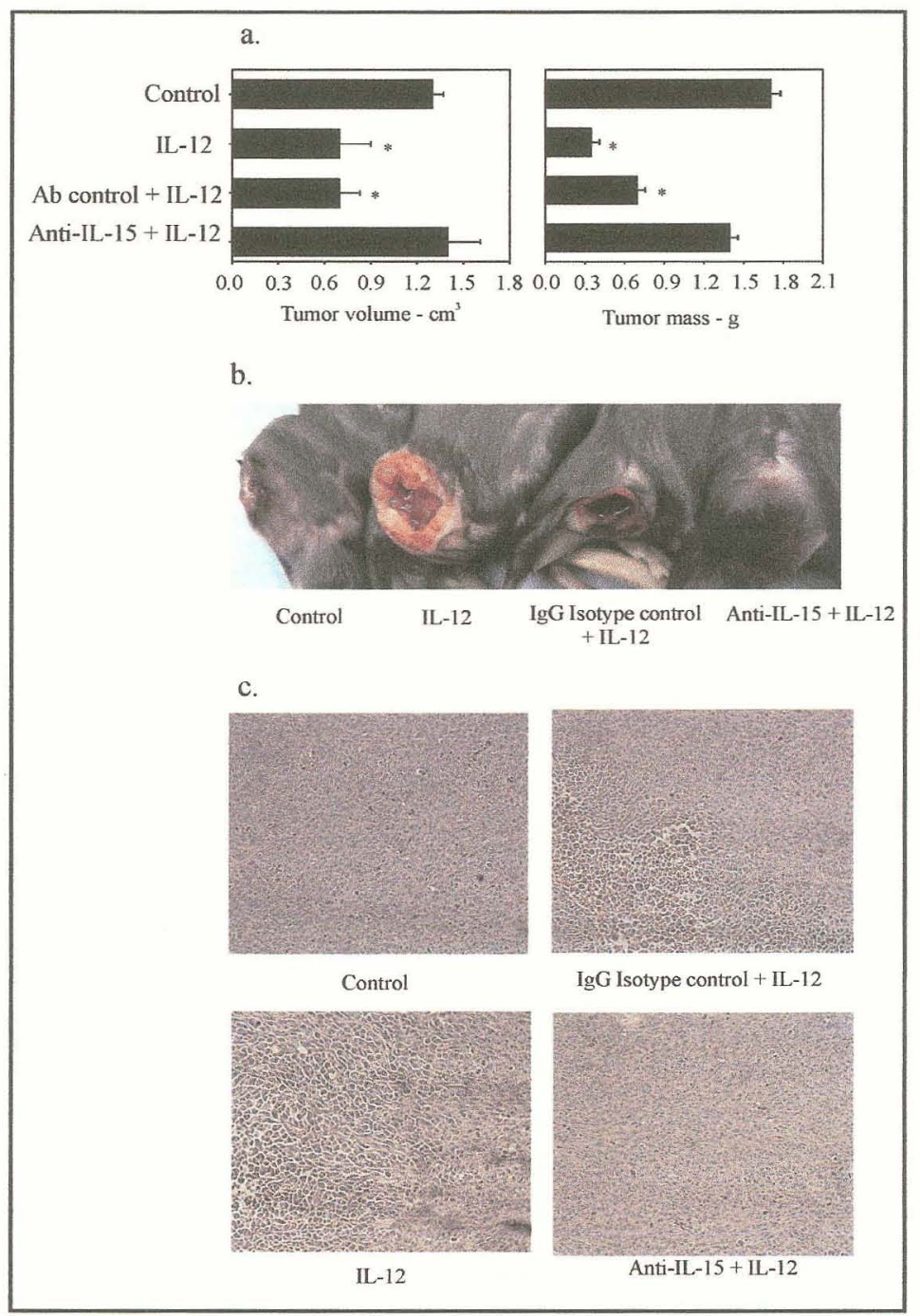

Figure 16. Neutralizing IL-15 prevents IL-12 induced tumor destruction. Tumor bearing mice were injected with placebo or IL-12 encapsulated microspheres. One group was injected with $250 \mathrm{ng}$ anti-IL-15, $1 \mathrm{hr}$ prior to IL-12 treatment. The tumor mass was then harvested 10 days post injection for analysis. a) Tumor volume was measured using bisecting calipers and wet weights were acquired for comparison of the groups. Data are displayed as mean weight \pm s.d. of 5 mice. b) Tumor ulceration shown by comparison of digital images, c) H\&E stains from tumor cross sections were performed by University of Louisville Pathology Dept. Results represent 5 mice per group. 


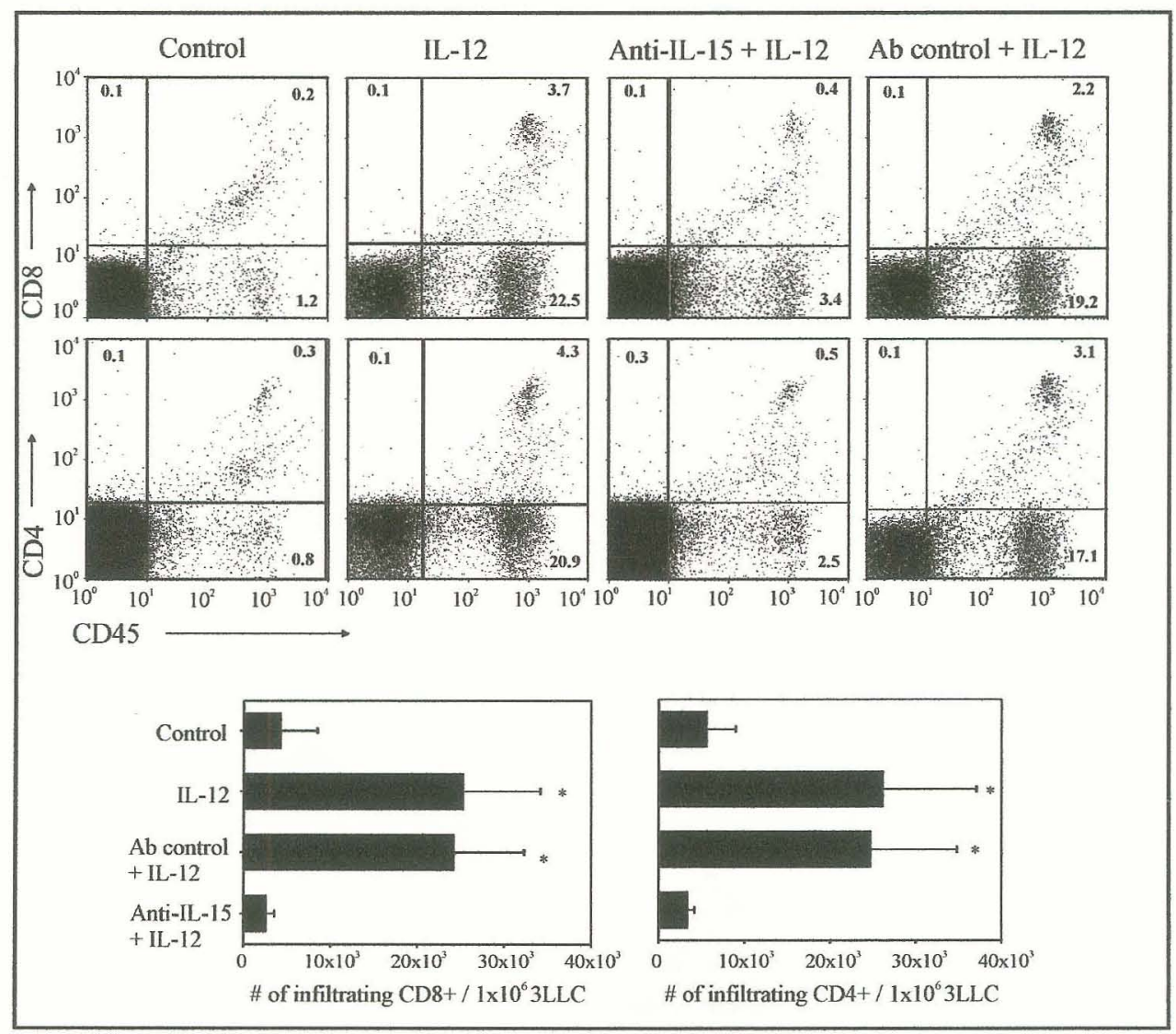

Figure 17. Neutralization of IL-15 reduces T cell infiltration into metastatic tissue in response to IL-12 treatment. Tumor bearing mice were injected with placebo or IL-12 encapsulated microspheres. One group was injected with $250 \mathrm{ng}$ anti-IL-15, $1 \mathrm{hr}$ prior to IL-12 treatment. The lungs were then harvested and analyzed for infiltrating cells 3 and 5 days after IL-12 injection. Data are expressed as the mean \pm s.d. of 5 mice per group, $* p$ $<0.05$ comparing control group or anti-IL15 group to IL-12 or IL-12 + isotype control treated groups. Results represent 2 independent trials. 


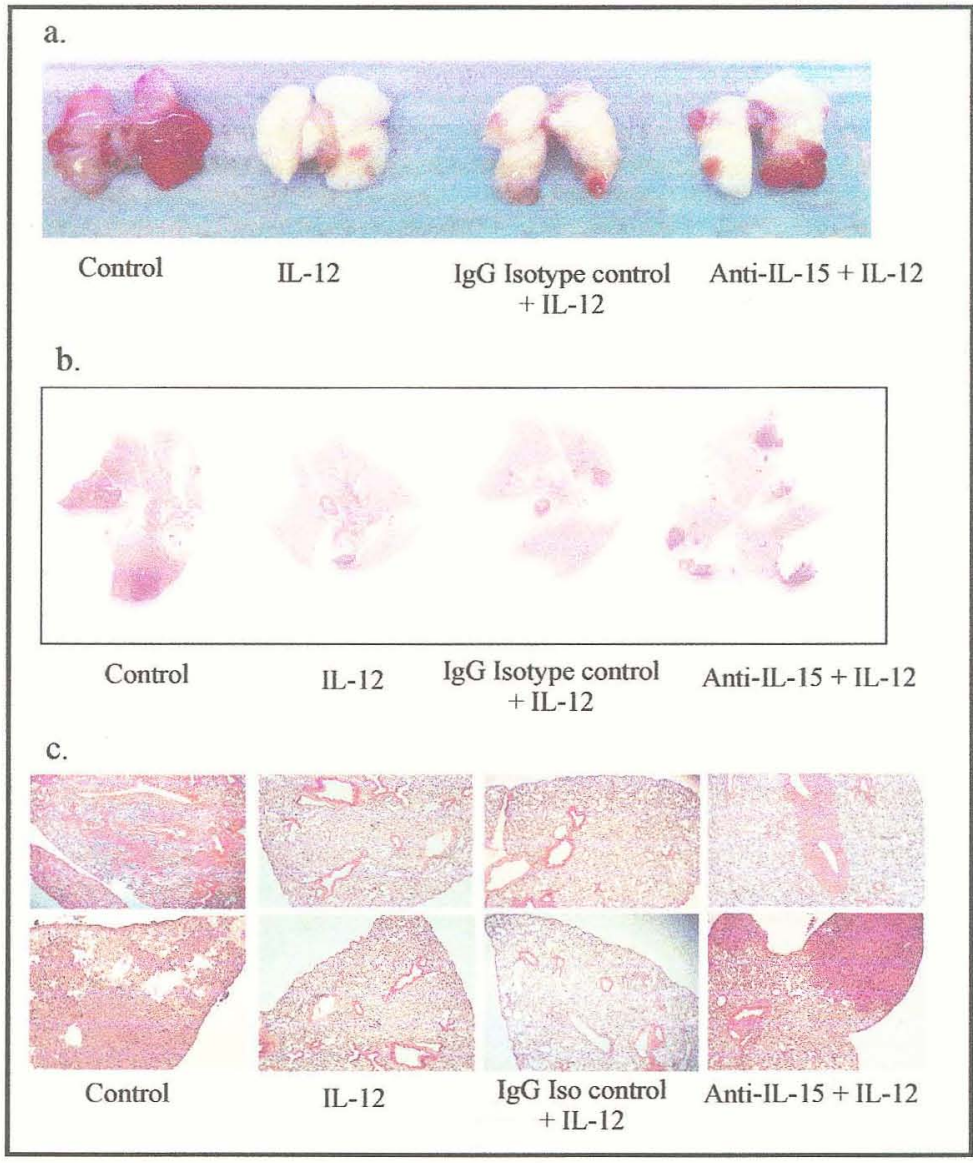

Figure 18. IL-15 neutralization prevented the clearance of tumor metastasis in the lung. Tumor bearing mice were injected with placebo or IL-12 encapsulated microspheres. One group was injected with $250 \mathrm{ng}$ anti-IL-15, $1 \mathrm{hr}$ prior to IL-12 treatment. The lungs were then harvested and analyzed 10 days after $\Pi \mathrm{L}-12$ injection for size and number of metastatic lesions. a) The number of lesions were counted as shown in digital image, b) cross sections of the lung were scanned to visualize extent of metastasis, c) an H\&E stain was done by University of Louisville Pathology Dept, to determine extent of metastasis, hemorrhage and lymphocyte infiltration. Similar results were observed in each of 5 mice per group. 
In addition to stimulating an anti-tumor immune response, IL-12 treatment may also be effective in the break-down of the vascular network supporting the tumor. Upon examination of IL-12 induction of the breakdown of the tumor stroma, we observed that tumor bearing animals treated with IL-12 displayed a dramatic reduction in size of the main blood vessel nourishing the tumor or appeared to be absent in many of the IL-12 treated mice. However, mice injected with neutralizing anti-IL-15 prior to IL-12 treatment were comparable to untreated control mice in that the major tumor blood vessel remained exuberant (Fig. 19).

\section{Macrophages in the tumor microenvironment are activated by cell contact with} tumor cells and tumor exosomes.

IL-12 treatment induces both production of inflammatory cytokines as well as release of intracellular IL-15. However, while IL-12 can modulate macrophage function, it can not effectively activate macrophages. Therefore, macrophages that responded to IL-12 injection presumably received an activating stimulus from some other source, e.g.NK cells that were activated in response to the IL-12 treatment, the combination of tumor produced cytokines or cell contact with the tumor.

To determine whether NK cells activated by IL-12 were responsible for stimulating macrophage activation, macrophages were harvested from beige mice, which are reported to lack functional NK cells, bearing a subcutaneous tumor or tumor free mice. Cytokine production and gene expression were then measured by cytokine bead array (CBA) or qRT-PCR. Cytokine production TNF $\alpha$, MCP-1, IL-10 and IL-6 


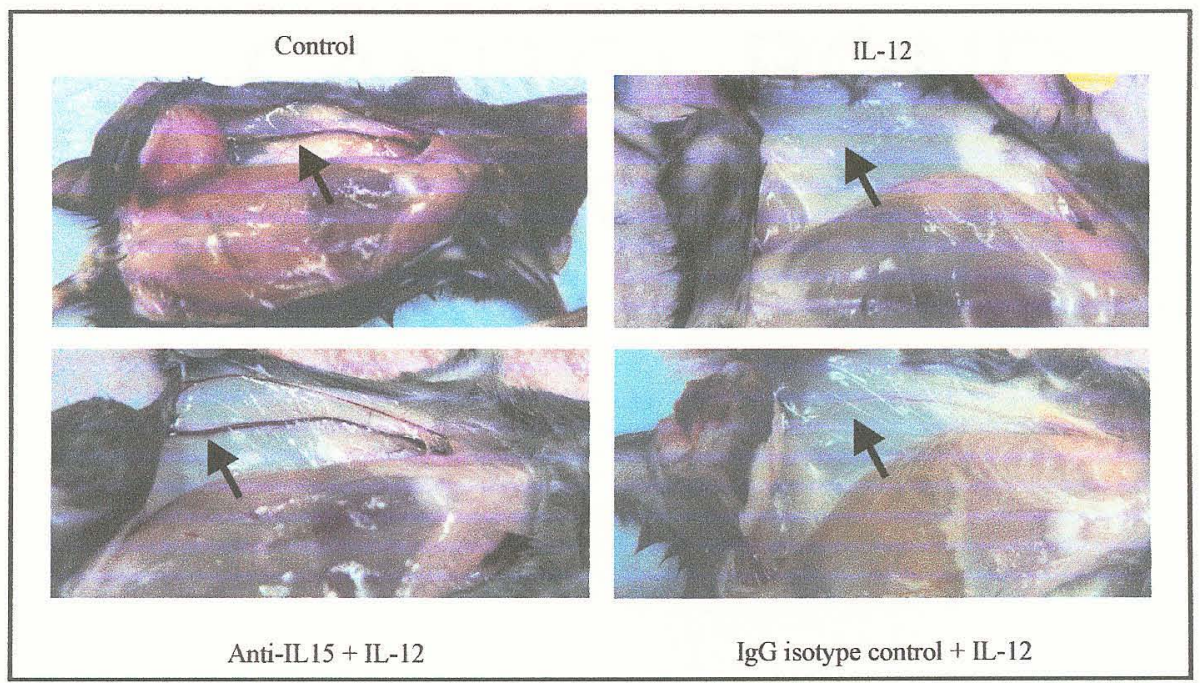

Figure 19. IL-12 treatment prevents formation or induces the break-down of the major tumor blood vessel, which is prevented upon neutralization of IL-15. Tumor bearing mice were injected with placebo or IL-12 encapsulated microspheres. One group was injected with $250 \mathrm{ng}$ anti-IL-15, $1 \mathrm{hr}$ prior to IL-12 treatment. Mice were euthanized 10 days after IL-12 treatment for analysis of destruction of the tumor and metastasis. Images of the tumor blood vessel are representative of 5 mice per group. 
were elevated in beige TAMs compared to macrophages from tumor free beige mice upon LPS stimulation. However, TAM production of IL- 6 and TNF $\alpha$ was further amplified after IL-12 injection and IL-10 and MCP-1 cytokine production was reduced compared to placebo treated. Additionally, cytokine genes MIF and TGF $\beta$ where found to be upregulated in beige TAMs but were significantly reduced upon IL-12 treatment. Furthermore, pro-inflammatory IL-15 mRNA was dramatically increased in response to IL-12 in both normal macrophage and TAMs (Fig. 20). In addition to mRNA expression, IL-15 protein was measured at ng levels in the serum of tumor bearing beige mice after treatment with IL-12, indicating activated NK cells are not essential for activation of macrophage cytokine production in the initiation phase of the IL-12 induced anti-tumor response. To confirm that macrophage release of IL-15 in response to IL-12 treatment could continue to contributed to the observed CD45+/CD8+ lymphocyte infiltration in the absence of NK cells, NK cells were depleted from normal and beige mice by antiNK1.1 24 hrs prior to IL-12 treatment, additionally to one group neutralizing anti-IL-15 was administered $1 \mathrm{hr}$ prior to $\mathrm{IL}-12$ injection. Depleting NK1.1 $1^{+}$cells did not inhibit CD8 + T cell infiltration in response to IL-12 induced IL-15 release. Infiltration was actually slightly increased in the tumor in the absence of NK cells (Fig.21).

The demonstrations that 1) IL-12 does not activate expression of function in normal resting macrophages, 2) that IL-12 does induce strong inflammatory functions in TIMs and TAMs and 3) that NK cells are not essential to the activation of TIMs and TAMs, suggests that TIMs and TAMs were activated prior to IL-12 treatment. Several investigators have reported evidence that TIMs and TAMs are in an activated state and 


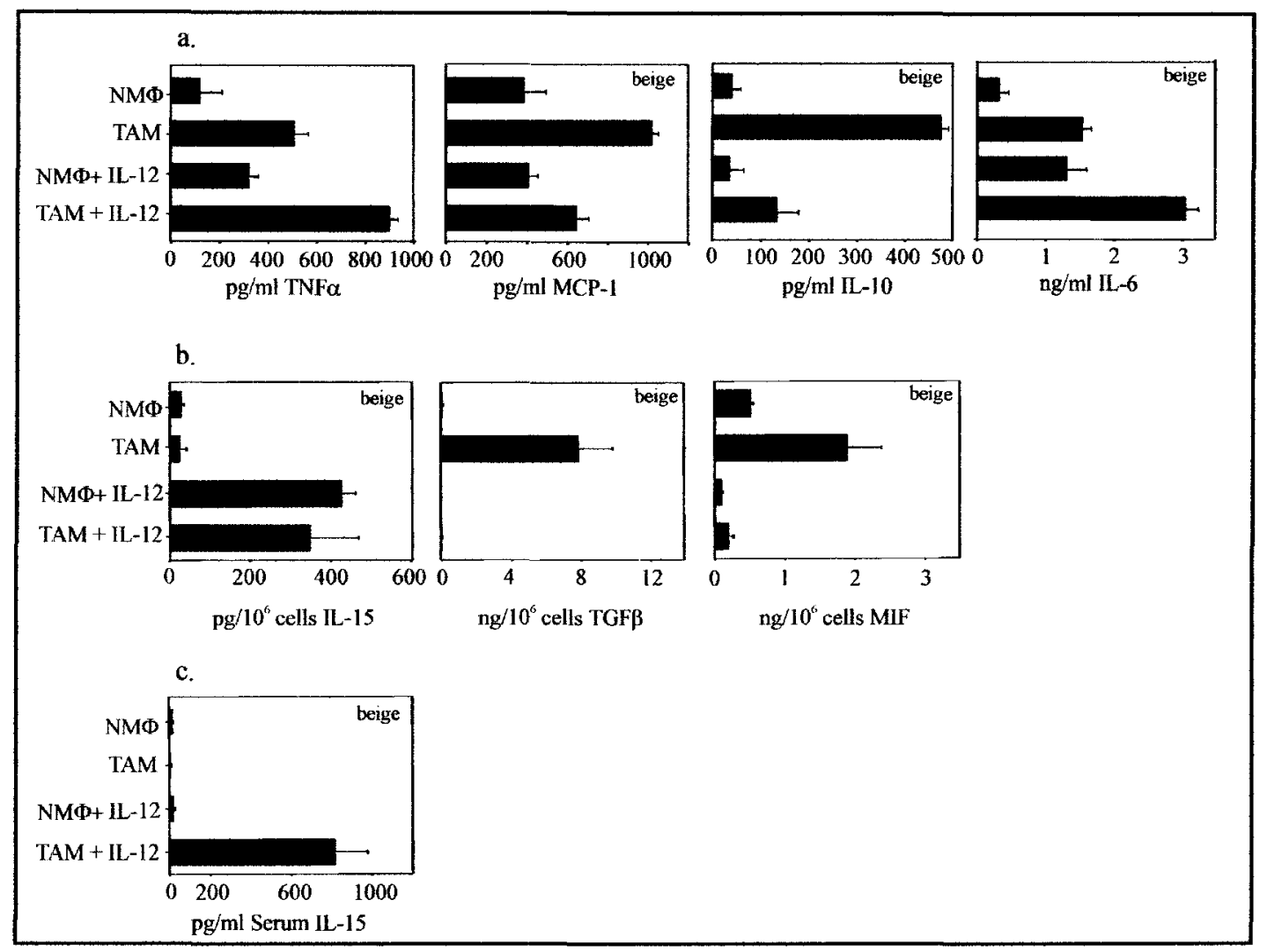

Figure 20. Macrophages in beige mice respond to IL-12 treatment. Macrophages were harvested from the spleen of tumor bearing beige mice before or 3 hours post IL-12 injection. Cells were either a) plated for analysis of TNF $\alpha$, MCP-1, IL-10 and IL-6 cytokine production in response to LPS or b) lysed immediately for mRNA extraction to assay cytokine gene expression by qrt-PCR. c) A blood sample was also taken to measure serum IL-15 levels by ELISA. Results are displayed as mean \pm s.d. of 3 mice. Cytokines TNF $\alpha$, MCP-1, IL-10 and IL-6 are from a single trial of 3 separate mice, Gene expression of MIF, TGF $\beta$, and IL-15 are representative of 3 independent trials of 3 separate mice, and serum IL-15 levels are representative of 2 independent trials of 3 separate mice. 

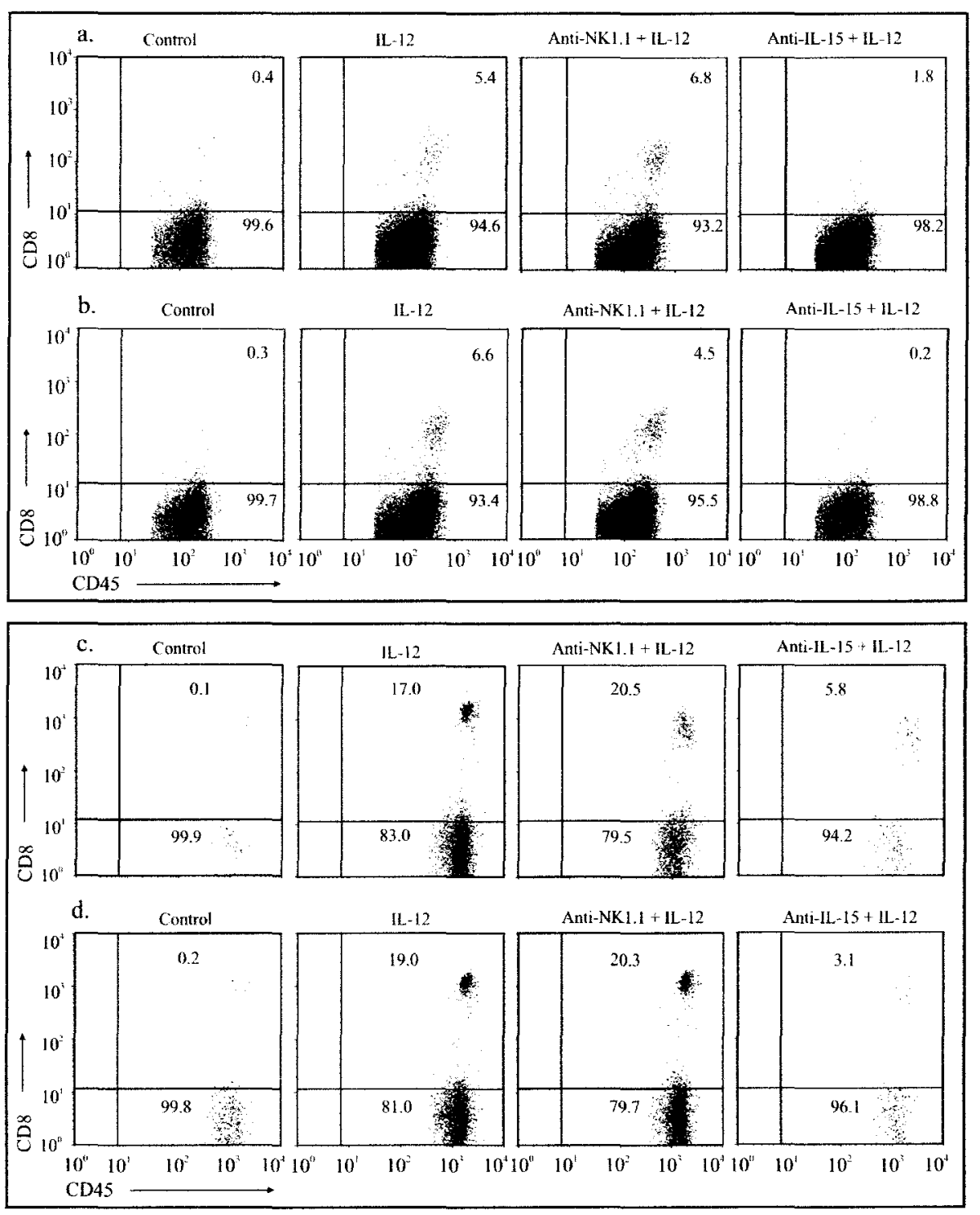

Figure 21. Depleting NK cells does not inhibit $\mathrm{T}$ cell infiltration in response to IL-12 induced IL-15 release. Tumor bearing beige $(\mathrm{a}, \mathrm{c})$ or tumor bearing wild type $(\mathrm{b}, \mathrm{d})$ were depleted of NK cells by injection of anti-NK1.1 24 hrs prior to IL-12 treatment. Anti-IL15 was injected $1 \mathrm{hr}$ prior to IL-12 injection. The tumors $(\mathrm{a}, \mathrm{b})$ and lungs $(\mathrm{c}, \mathrm{d})$ were harvested 5 days after IL-12 treatment to analyze infiltrating CD8+ T cells. Percentages displayed are based on percent of CD45+. Results are representative of 2 independent trials of 4 mice per group. 
express functions which promote angiogenesis and tumor growth. The mechanism of this activation, especially of the tumor-distal TAMs remains unresolved ${ }^{26,31,32,35,36,48}$. To corroborate that macrophages were in as activated state in vivo in the 3LLC model, TIMs and TAMs were purified from the primary tumor mass, or from the spleen, peritoneal cavity, and lung tissue of mice bearing subcutaneous 3LLC tumors and lung metastasis. The macrophages were plated for $17 \mathrm{hrs}$ without exogenous stimulation after which the cultures were assayed for cytokine accumulation. TAMs from the spleen, peritoneal lavage, and the lung produced 2-16 times more TNF $\alpha, \mathrm{MCP}-1, \mathrm{IL}-10$, and IL-6 than macrophages from normal healthy tissues. TIMs also produced elevated levels of TNF $\alpha$, MCP-1, and IL-6 but did not produce detectable levels of IL-10 (Fig.22a). These results were confirmed in other lung carcinoma models, LL2 and TC-1, indicating that macrophages are activated in the presence of a tumor and the results observed were not unique to the 3LLC carcinoma (Fig.22b).

To determine whether cytokines or other soluble factors released by the tumor were responsible for macrophage activation in situ, macrophages from normal mice were cultured in vitro with the filtered supernatant from primary $3 L L C$ tumor cell cultures. The 3LLC supernatant contained high levels of MCP-1, VEGF, and TGF $\beta$. Cytokine analysis did not reveal evidence of macrophage activation with the exception of pro-MMP-9, which has been shown to be released in response to high levels of VEGF ${ }^{35}$, ${ }^{37}$. However, culturing the macrophages in unfiltered tumor supernatant clearly resulted in activation as measured by an increase in production of TNF $\alpha$, MCP-1, IL-10, IL-6, VEGF, and pro-MMP-9 (Fig.23). Previous reports have identified tumor released 


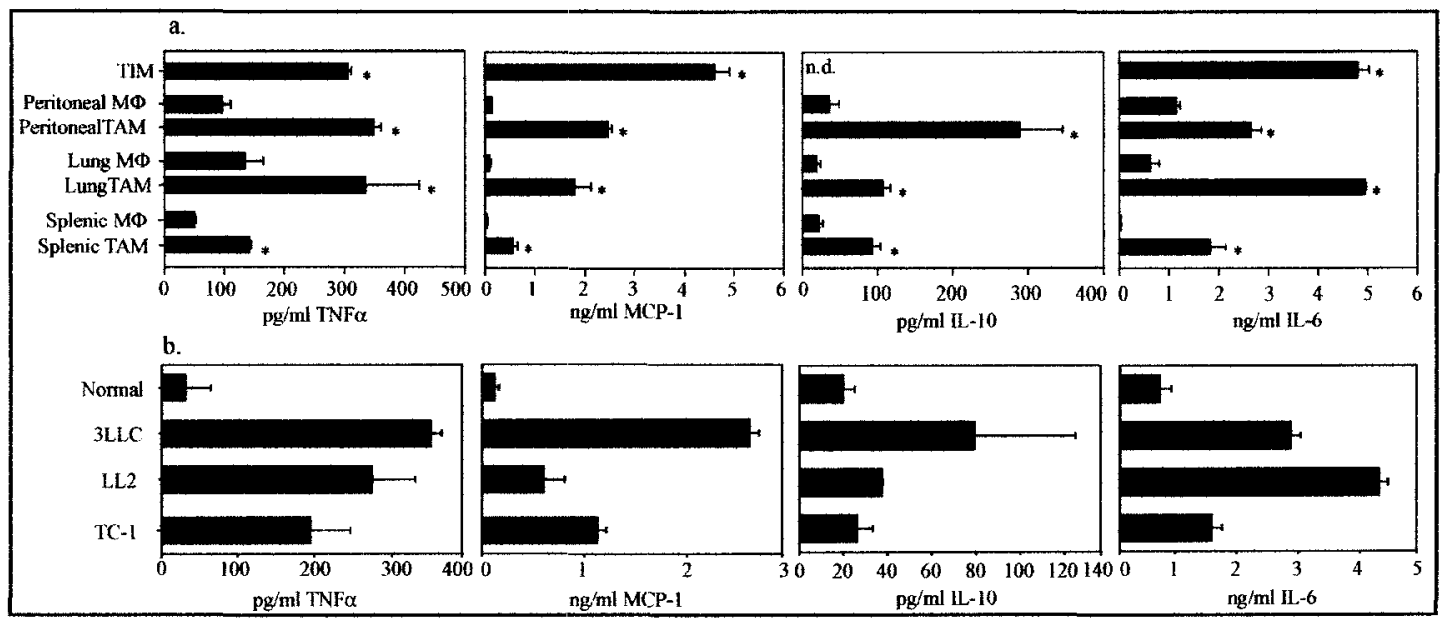

Figure 22 - TAMs are actively producing cytokines upon ex vivo analysis. MФ were purified from the primary 3LLC tumor, peritoneal lavage, spleen, and lungs of normal or mice bearing a $0.6 \mathrm{cc}$ tumor with lung metastasis..(a) TIMs and TAMs were plated overnight with no further stimulation for 17 hours to allow for cytokine accumulation. Supernatants were then harvested and assayed by CBA. (b) TAMs from various tumor models were harvested and assayed for cytokine production. Data represented as mean + s.d., $\mathrm{n}=3$ mice . ${ }^{*} \mathrm{p}<0.0001$ comparing TAMs vs macrophages from control animals. 


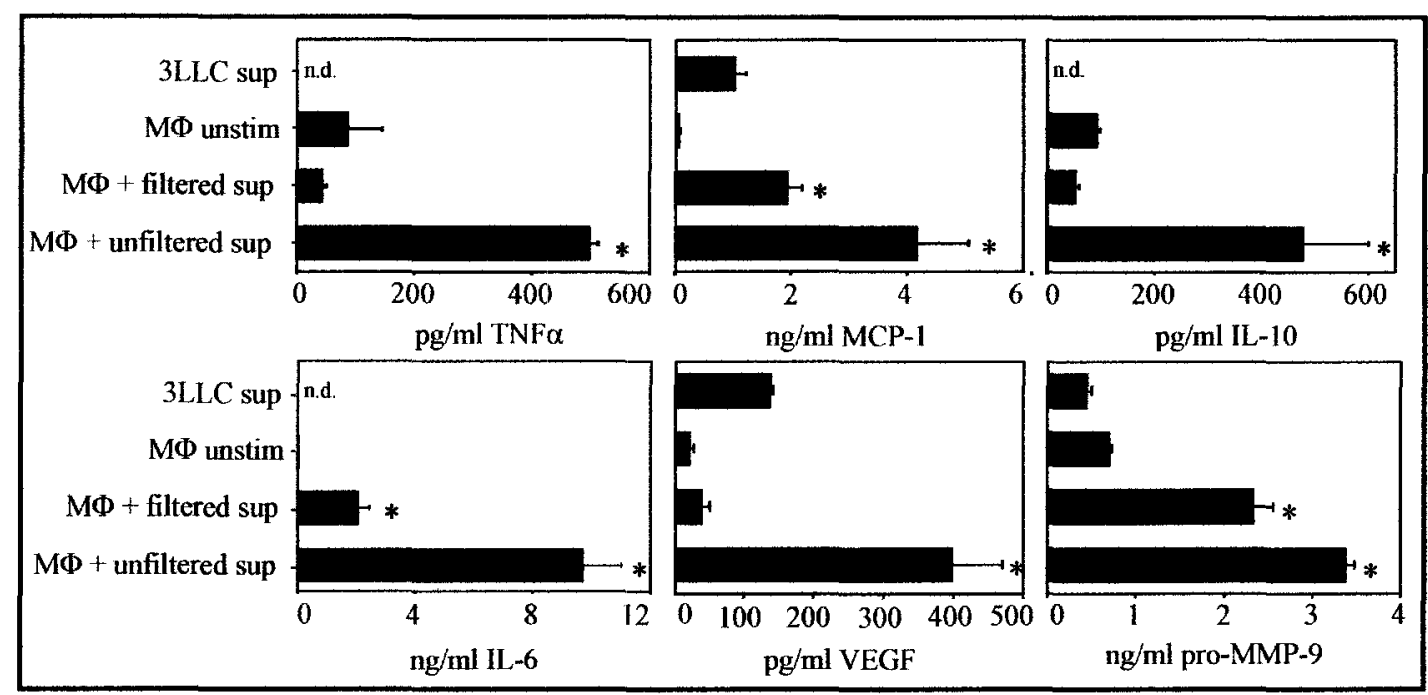

Figure 23. Unfiltered tumor supernatant activated macrophages. Normal peritoneal MФs were cultured overnight with filtered $(0.2 \mu)$ or unfiltered supernatants of $3 L L C$ tumors passaged one day ex vivo. The culture supernatants were assayed for cytokines. Data is expressed as mean + s.d. of triplicate cultures. n.d. $=$ none detected. Results are representative of 5 independent trials. 
exosomes of the tumor membrane expressing tumor cell surface molecules ${ }^{54}$. The exosomes are thought to contribute to the systemic suppression of the immune system as well as in tumor metastatic development ${ }^{54,55,90,91}$. This led us to consider the possibility that exosomes in the unfiltered tumor culture supernatant could activate macrophages upon cell contact. To determine whether exosomes released by the tumor were capable of activating macrophages, exosomes were purified by magnetic bead selection using a tumor specific hMuc-1 Ab and were cultured with macrophages for $17 \mathrm{hrs}$. ELISA and CBA analysis confirmed that normal peritoneal macrophages cultured with exosomes produced elevated levels of TNF $\alpha$, MCP-1, IL-10, IL-6, VEGF, and pro-MMP-9 (Fig.24).

\section{Tumor cell and macrophage interactions occur through CD40/CD154 and NKG2D/Rae-1.}

Previous studies on many types of cancer reported that tumor cells express activation receptors and their corresponding ligands such as CD40/CD154 and NKG2D/Rae-1 20,53,56,92,93. We have confirmed that these ligands are expressed by 3LLC ex vivo (Fig 25). Macrophages are known to be activated by ligation of CD40 ${ }^{12,57}$ and macrophages purified from tumor bearing mice displayed elevated expression of CD40 compared to macrophages from tumor-free mice (Fig.25). Analysis of macrophage surface expression of NKG2D produced varying results when assayed by flow cytometry, 3 trials indicated elevated NKG2D surface expression on peritoneal 


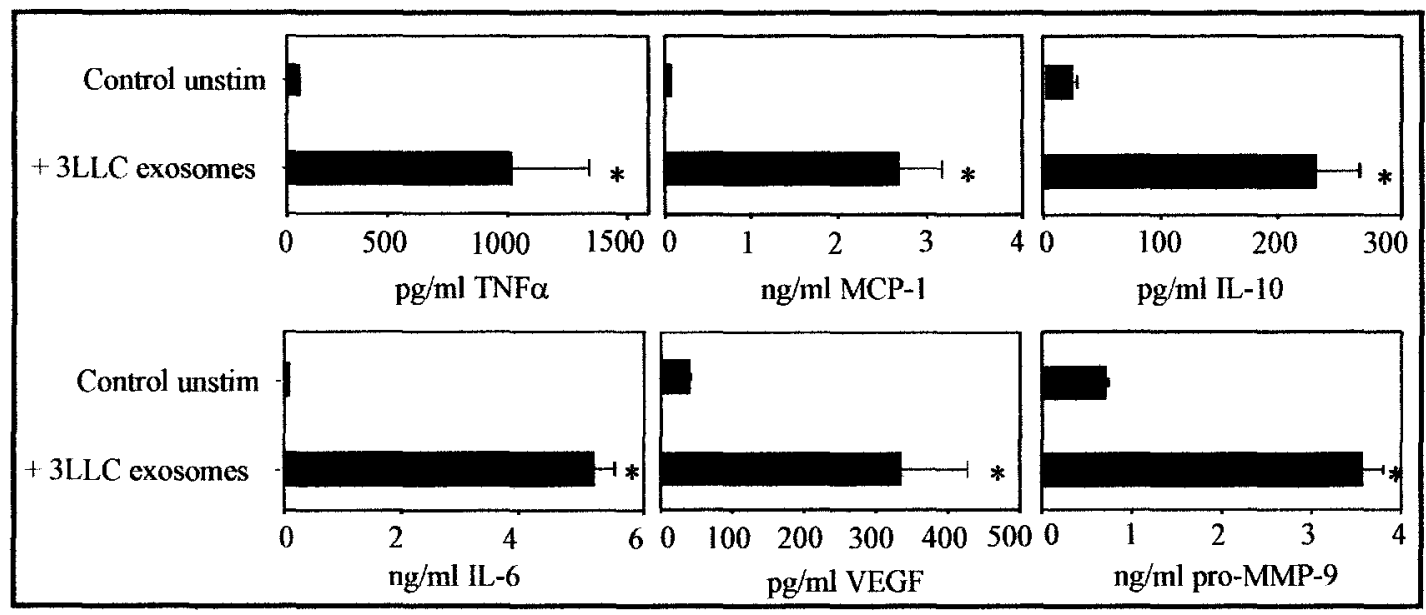

Figure 24. Tumor exosomes activate MФs. Normal peritoneal MФs were cultured overnight with exosomes purified by magnetic bead selection of hMuc-1+ particles in unfiltered supernatants of cultures of hMuc-1 transfected 3LLC tumor cells. Data are expressed as mean \pm s.d. of triplicate cultures. Results are representative of 4 independent trials. 


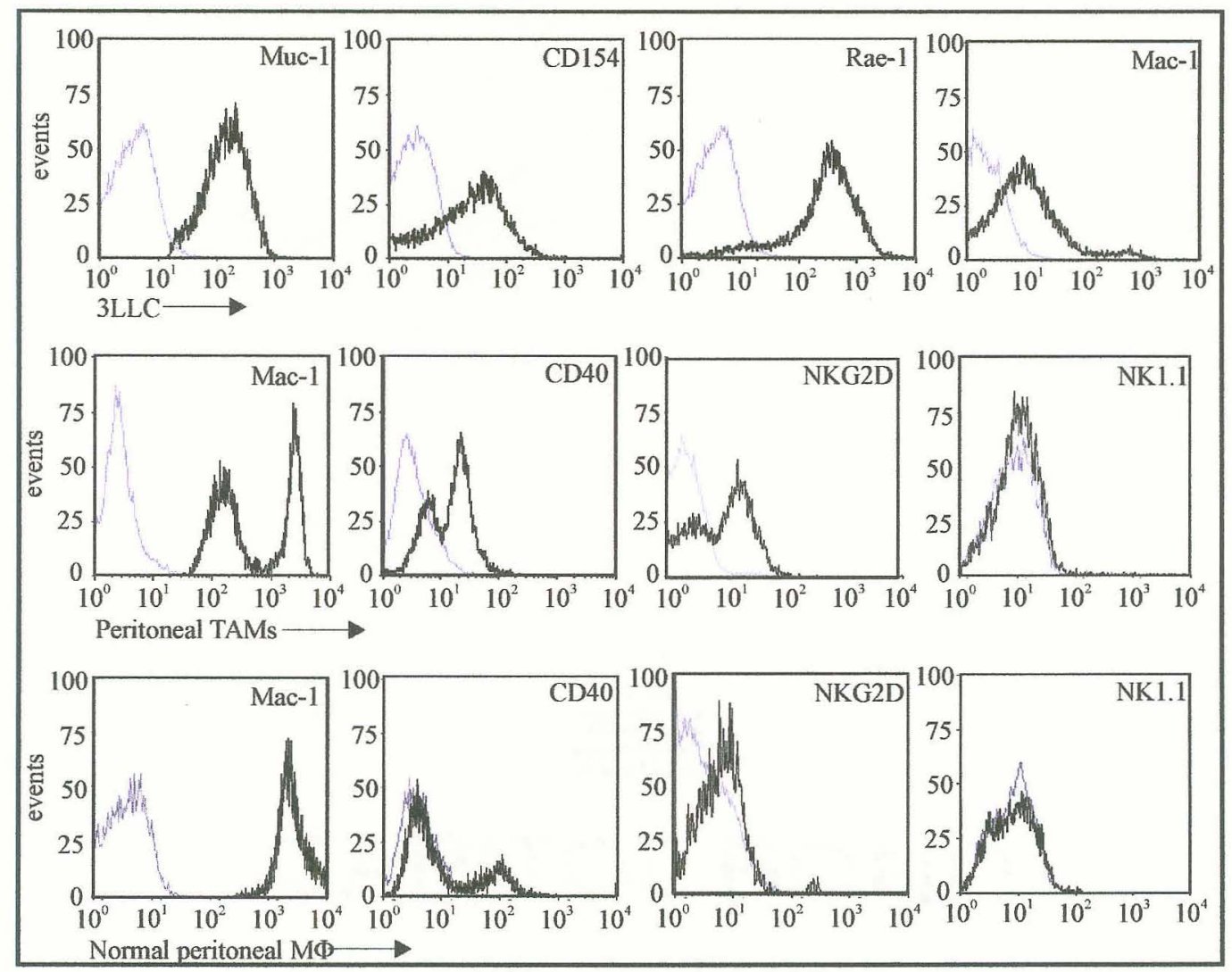

Figure 25. Tumor cells express CD40L and Rae-1 and TAMs but not normal MФ express elevated levels of surface CD40 and NKG2D receptors. 3LLC as determined by hMuc-1+ population were stained ex vivo for cell surface expression with fluorescent Abs to CD40L (CD154) and Rae-1. MФs as determined by the Mac-1 + (CD11b) population were stained ex vivo for cell surface expression of CD40 and NKG2D. Staining for NK1.1 was used as a control to verify populations were free of NK contamination. Results are representative of 3 independent trials. 
TAMs in comparison to normal peritoneal macrophages, while 2 trials displayed only background staining levels. The data was even more disconcerting by the observation that both TAM and normal macrophages from lung tissue stained positive for NKG2D surface expression, by others in our lab (data not shown). However the presence of NKG2D mRNA was observed in both TAM and normal macrophages by qrt-PCR, NKG2D gene expression was observed to be up-regulated by a factor of 78 in peritoneal TAMs in comparison to normal peritoneal macrophages and up-regulated by a factor of 32 compared to normal resting NK cells purified from a spleen of a healthy animal (Fig.26). Interestingly, normal peritoneal macrophages cultured for $24 \mathrm{hrs}$ in the presence of 3LLC exosomes and culture supernatant displayed increased NKG2D gene expression in comparison to normal macrophages cultured without exosomes (Fig. 26). To determine whether 3LLC exosomes were activating macrophages by ligation of CD40 or NKG2D, normal peritoneal macrophages were stimulated with 3LLC exosomes in the presence or absence of blocking anti-CD154 and/or anti-NKG2D. Blocking either CD154 or NKG2D significantly reduced exosome induced TNF $\alpha$, IL-10, IL-6, and proMMP-9 production. Blocking both NKG2D and CD40 ligation completely abrogated cytokine release including MCP-1, which was not significantly affected by blocking only one receptor ligand interaction (Fig 27). A titration of the Abs shows a dose dependant response when added separately, but total blockage of cytokine production or release when added in combination (Fig. 28). To insure specific blocking by anti-NKG2D, rmNKG2D-Fc was added to the cultures. The addition of rmNKG2D-Fc prevented inhibition of cytokine production by anti-NKG2D (Fig 29). Further analysis revealed that, wild type and 


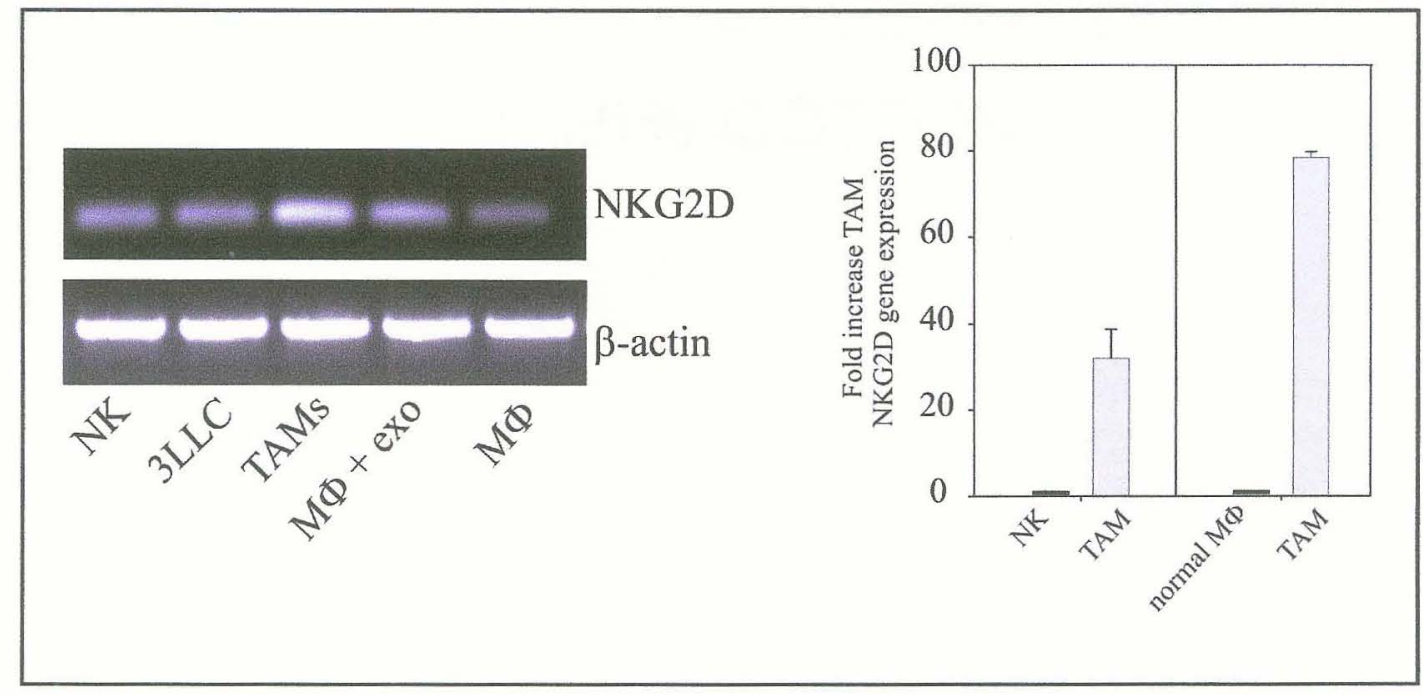

Figure 26. TAMs express elevated NKG2D gene expression. Macrophages were harvested from normal or tumor bearing mice and lysed for mRNA extract immediately or normal macrophages were plated with exosomes for $24 \mathrm{hrs}$ prior to lysis. NK cells were harvested from the spleen of a normal mouse and used as a positive control for NKG2D expression. 3LLC tumor cells were also lysed ex vivo for comparison. Results are representative of 4 independent trials. 


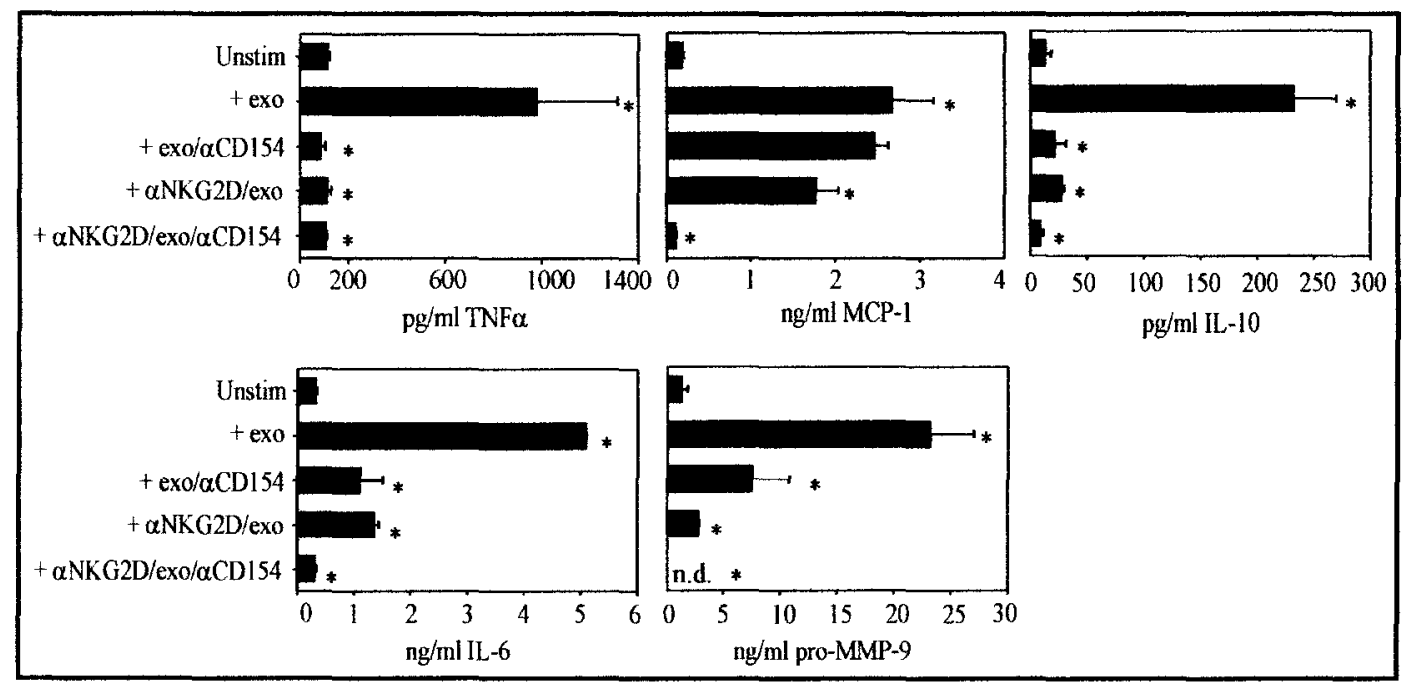

Figure 27. CD40 and NKG2D are involved in macrophage activation by tumor exosomes. Macrophages were purified from the peritoneal lavage of normal animals and cultured for $17 \mathrm{hrs}$ with 3LLC exosomes in the presence or absence of blocking Abs to NKG2D and CD154. The supernatants were then harvested and analyzed for cytokine production by CBA and ELISA. Results are displayed as mean \pm s.d. of triplicate cultures. ${ }^{*} p<0.05$ comparing un-stimulated vs exosome stimulated and exosome stimulated vs exosome stimulated in the presence of blocking Abs. Results are representative of 3 independent trials. 


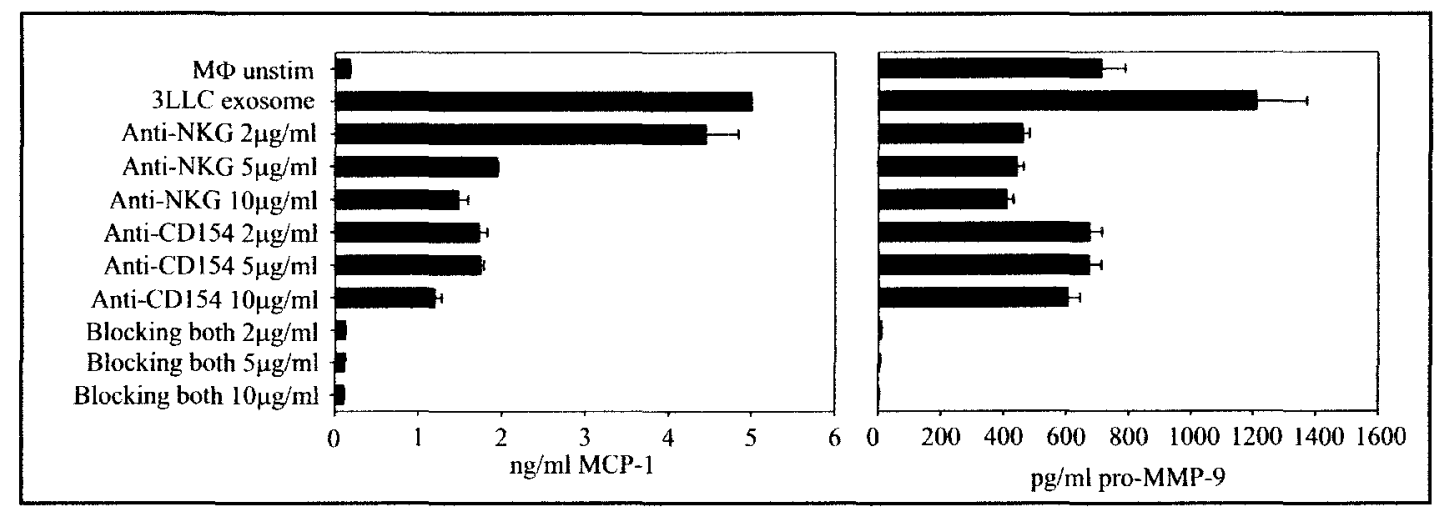

Figure 28. Blockade of either NKG2D or CD40 ligation interferes with exosome activation of MF. The combined inhibition of ligation of both CD40 and NKG2D was synergistic and completely abrogated the response of MF to 3LLC exosomes. Normal peritoneal MФ were plated in the presence or absence of 3LLC exosomes in increasing concentrations of anti-NKG2D and/or anti-CD154 Ab separately or in combination for 17 hrs. Culture supernatants were then harvested and analyzed by ELISA for cytokine production. Results are displayed as mean \pm s.d. of triplicate cultures and representative of two independent trials. 


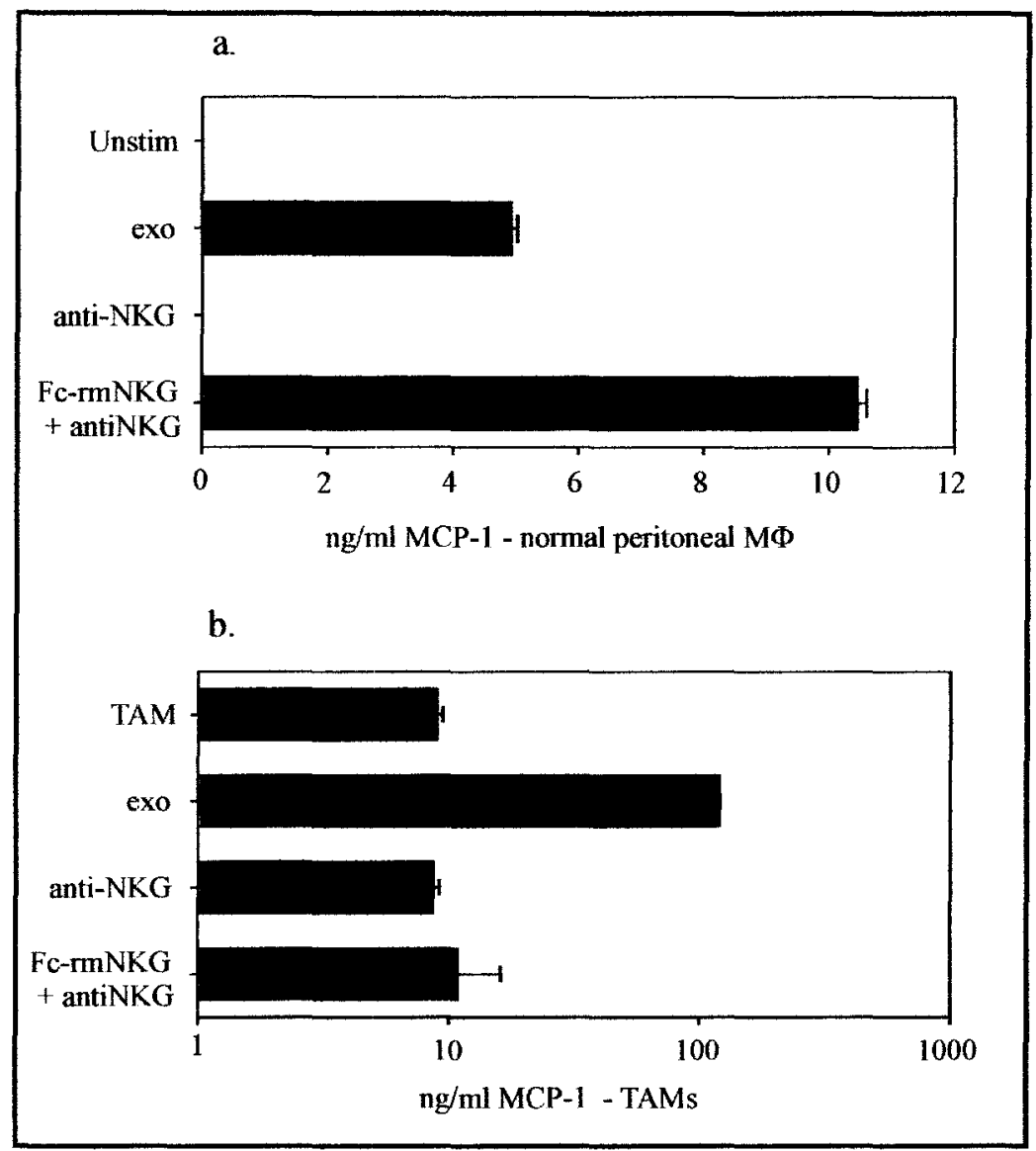

Figure 29. Anti-NKG2D Ab specifically blocks macrophage activation by exosome NKG2D ligation as shown Fc-rmNKG2D inhibited the ability of the blocking antibody to prevent cytokine production. Peritoneal macrophages were purified from (a) normal animals or (b) tumor bearing animals and cultured for $17 \mathrm{hrs}$ with 3 LLC exosomes in the presence or absence of blocking anti-NKG2D or Fc-rmNKG2D + anti-NKG2D. The supernatants were then harvested and assayed by ELISA. Data are displayed as mean \pm s.d. of triplicate cultures. The results are representative of 2 independent trials. 
CD40-/- macrophages cultured with exosomes for $17 \mathrm{hrs}$ were activated as measured by macrophage cytokine production. CD40-/- macrophages stimulated with exosomes were observed to produce substantial levels of MCP-1, IL-6, and pro-MMP-9 production in comparison to un-stimulated macrophages, but they produced significantly less TNF $\alpha$, MCP-1, IL-10, IL-6, and pro-MMP-9 than wild type macrophages stimulated with exosomes (Fig.30). In addition blocking NKG2D in CD40-/- macrophages completely abrogated TNF $\alpha$, MCP-1, IL-10, IL-6, and pro-MMP-9 production and/or release (Fig. 30). As a control it was shown that blocking Abs for NKG2D and CD154 did not prevent macrophage activation of cytokine production in response to LPS stimulation (Fig 31).

\section{CD40 is essential for metastatic growth.}

Macrophages have been shown to be important for tumor metastatic capabilities. They have been referred to as the mediators of the angiogenic switch in tumorigenesis by providing many cytokines and growth factors important for tumor escape and metastasis, particularly VEGF and MMP- $9^{26,30,37,94}$. Therefore to determine whether the failure of macrophage activation by CD40 ligation in vivo altered the activities of TAMs or tumor cells, 3LLC tumor cells were injected subcutaneously into wild type or CD40-/- mice and macrophages were harvested when the tumors were approaching $0.5 \mathrm{cc}$ to assay for cytokine production. Production of TNF $\alpha$, MCP-1, IL-10, and IL-6 were greatly decreased in the TAMs from CD40-/- animals compared to wild type TAMs (Fig.32). Interestingly, even the release of intracellular IL-15 upon exosome 


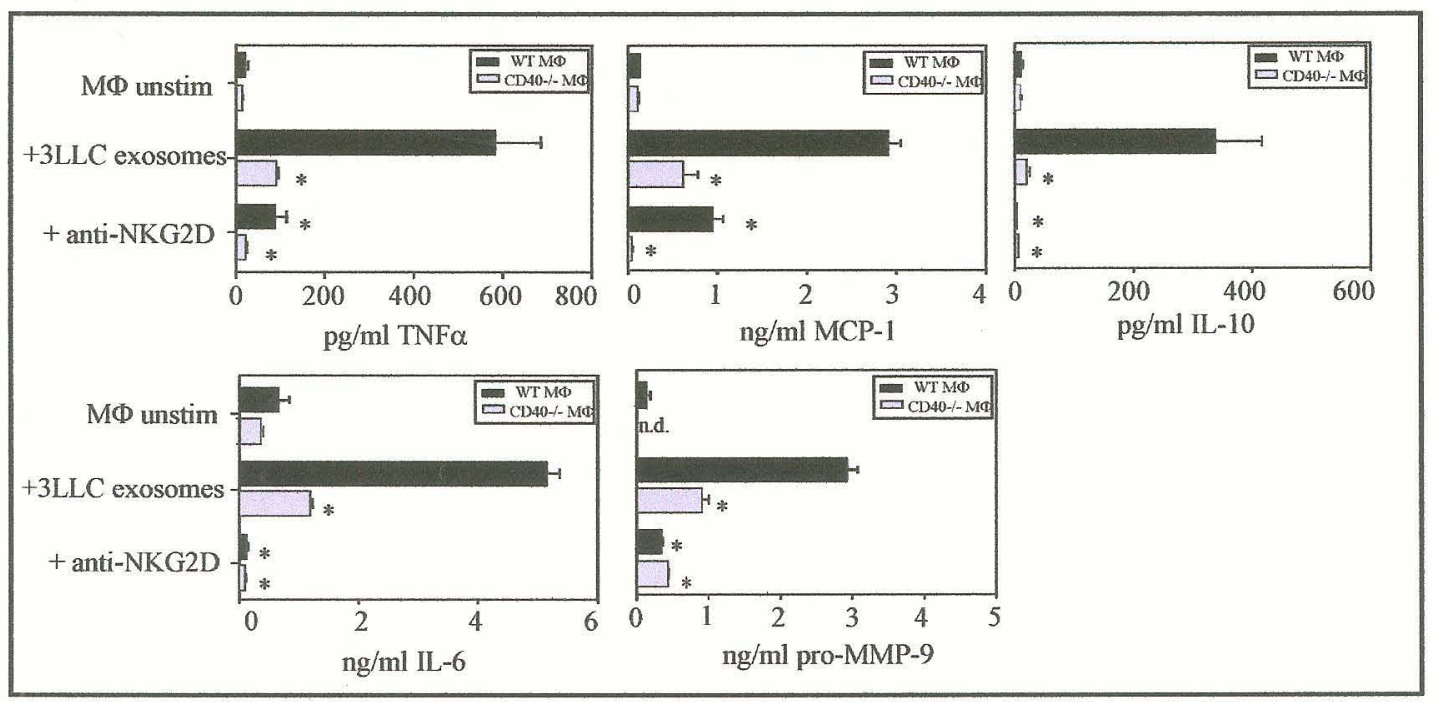

Figure 30. CD40 and NKG2D are involved in macrophage activation by tumor exosomes. Peritoneal MФs from normal or CD40-/- mice were cultured overnight with $3 \mathrm{LLC}$ exosomes in the presence or absence of neutralizing anti-NKG2D. The culture supernatants were assayed for cytokines by CBA and ELISA for MMP-9. Data are expressed as mean \pm s.d. of triplicate cultures $* p<0.05$ comparing groups WT MФs vs CD40-/- MФs stimulated by 3LLC exosomes. Data representative of 2 independent trials. 


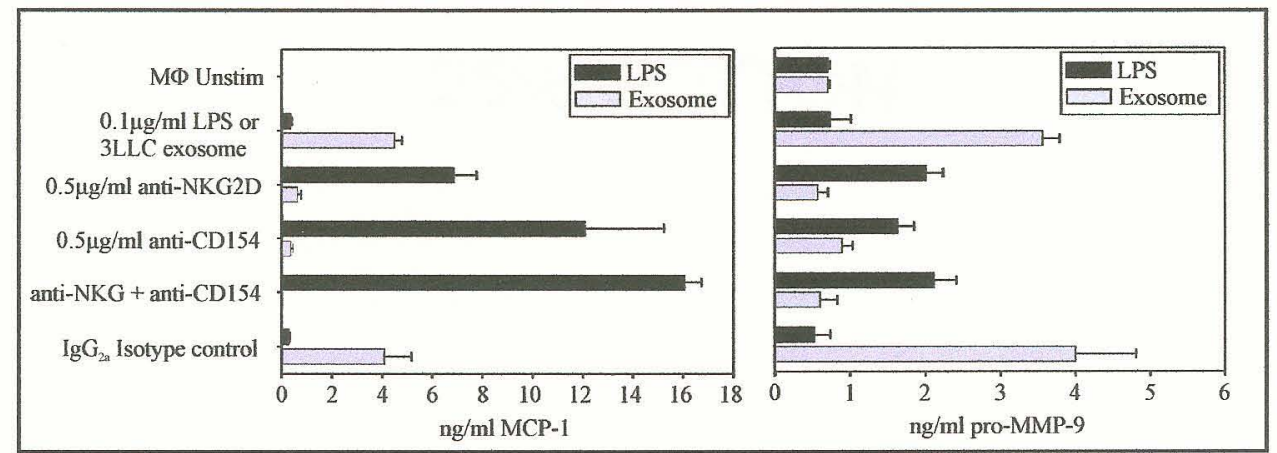

Figure 31. Blocking Abs to NKG2D and CD154 do not inhibit macrophage cytokine production in response to LPS stimulation. Macrophages were purified by bead selection from peritoneal lavage of normal mice. Cells were then stimulated with LPS or 3LLC exosomes in the presence or absence of anti-NKG2D and anti-CD154 blocking Abs for $17 \mathrm{hrs}$. The supernatants were then harvested and assayed by ELISA to detect cytokine production. Results are displayed as mean \pm s.d. of triplicate cultures. Results are representative of 2 independent trials. 


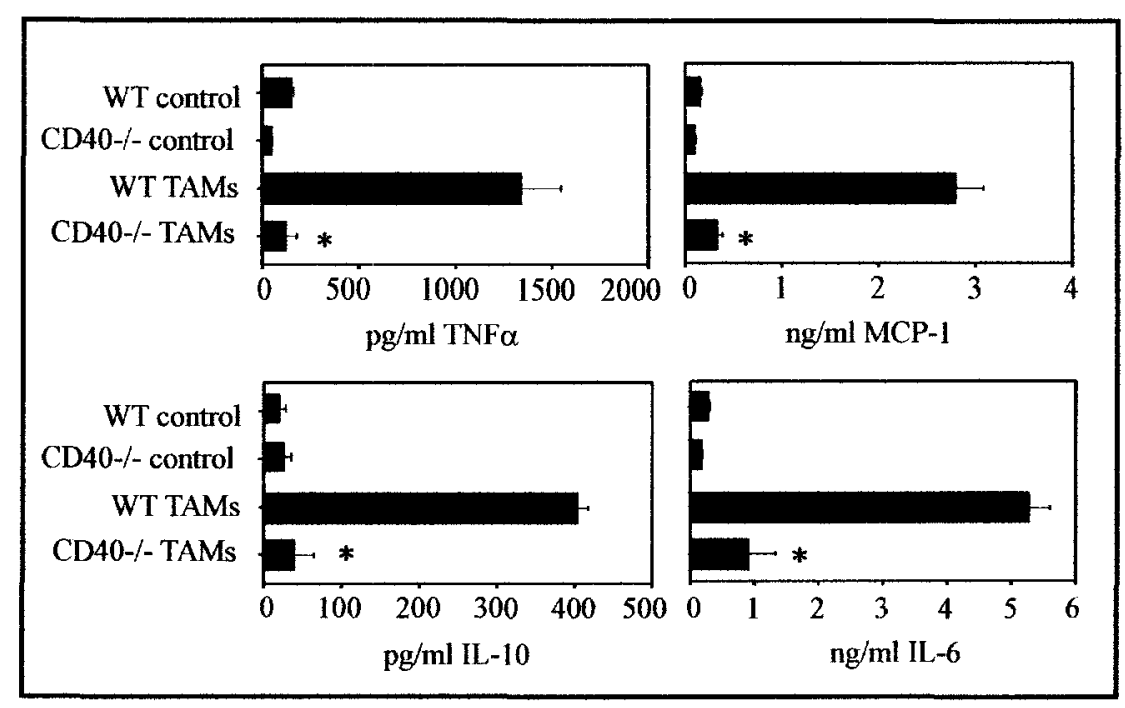

Figure 32. Cytokine secretion is decreased in TAMs from CD40-/-tumor bearing mice. Peritoneal MФs were purified from peritoneal lavage of wild type and CD40-/- tumor bearing mice and cultured overnight without stimulus to allow for cytokine accumulation. The supernatants were analyzed for cytokines by CBA. Data are expressed as mean \pm s.d. for 3 mice per group. ${ }^{*} p<0.05$ comparing groups wild type TAMs vs CD40-/- TAMs. Results are representative of 2 independent trials. 


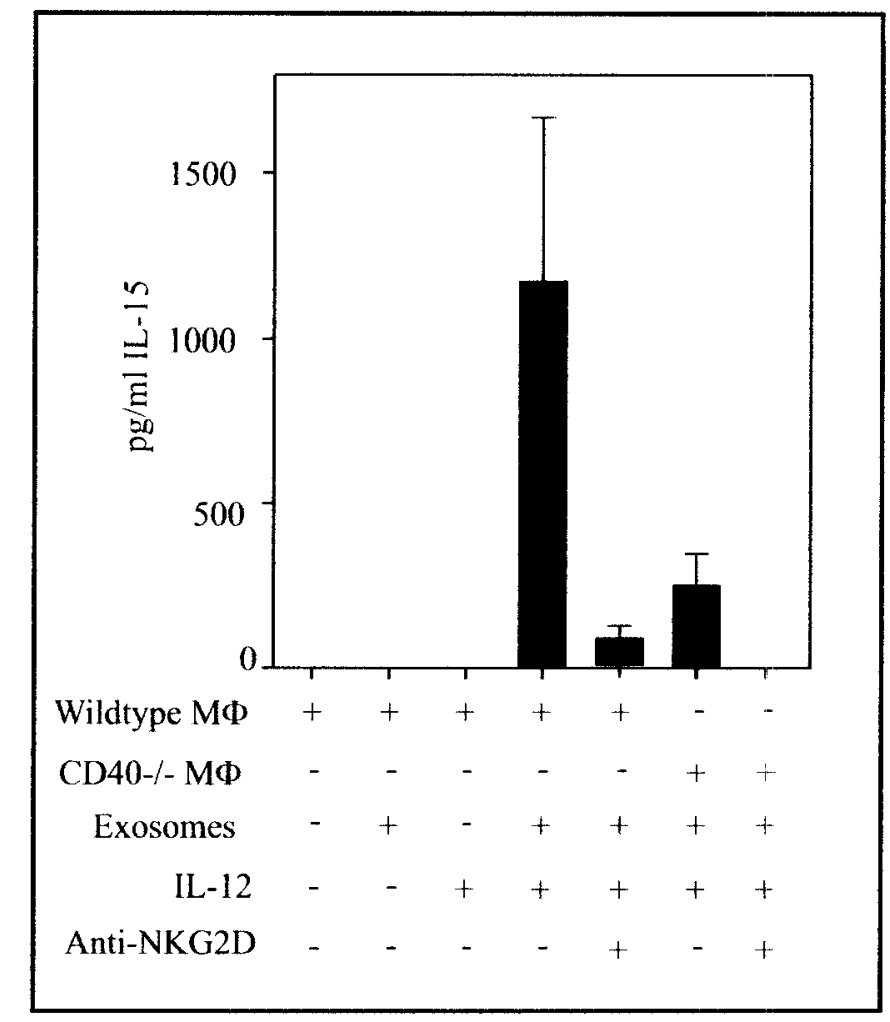

Figure 33. Tumor activated macrophage IL-15 release is blocked in the absence of CD40 and/or NKG2D ligation. Wild type or CD40 $\%$ peritoneal macrophages were cultured for $2 \mathrm{hrs}$ with exosomes $+5 \mathrm{ng} / \mathrm{ml} \mathrm{IL-12} \mathrm{in} \mathrm{the} \mathrm{presence} \mathrm{or} \mathrm{absence} \mathrm{of} \mathrm{anti-NKG2D.} \mathrm{IL-15}$ was assayed by ELISA. Data represented as mean $+\mathrm{s} . \mathrm{d} . \mathrm{n}=3$ mice, $\mathrm{p}=0.001$ for anti-NKG2D vs. wild type control, $p=0.0008$ for CD40-/- vs. wild type control, and $\mathrm{p}=0.0001$ for CD40-/ + anti-NKG2D vs. wild type control. n.d. $=$ no cytokine detected. Data representative of 2 independent trials. 
and IL-12 stimulation was blocked in the absence of functional CD40 or upon the addition of anti-NKG2D blocking Ab (Fig. 33). Conversely, although cytokine production and release by TAMs was inhibited in CD40-/- mice, growth of the primary tumor mass appeared to be more rapid. However, further observations revealed that unlike wild type animals there were no metastatic lesions in the lung tissue of CD40-/mice (Fig. 34), coinciding with a significant decrease in pro-MMP-9 and VEGF production by TAMs from CD40-/- animals (Fig. 35).

\section{TAMs stimulate tumor cells via CD40/CD154 interaction.}

The reduction in cytokine production was observed not only in the CD401- TAMs, but was also significantly decreased in tumor cells harvested from CD40-/animals in comparison to wild type mice (Fig.36). To determine whether this effect was due at least in part to reduced interaction with macrophages, the stability of tumor phenotype, which contributes to macrophage activation, was examined by flow cytometry. We observe that, 3LLC phenotype is not constitutive, but when cultured in vitro in the absence of the tumor stroma as well as the absence of macrophages or macrophage produced tumor supportive cytokines, the expression of surface receptors and ligands recedes (Fig.37a and Table 1). However, adding TAMs, but not normal macrophages, to cultures sustained or restored tumor expression of surface molecules, CD40, CD154,NKG2D, CD44, IL-15R $\beta$, Rae-1, and H2Kb (Fig.37b and Table 1). TAM support of tumor phenotype appeared to be dependent on several receptor ligand interactions, as indicated by the observation that TAMs from CD40-/- only were able to 


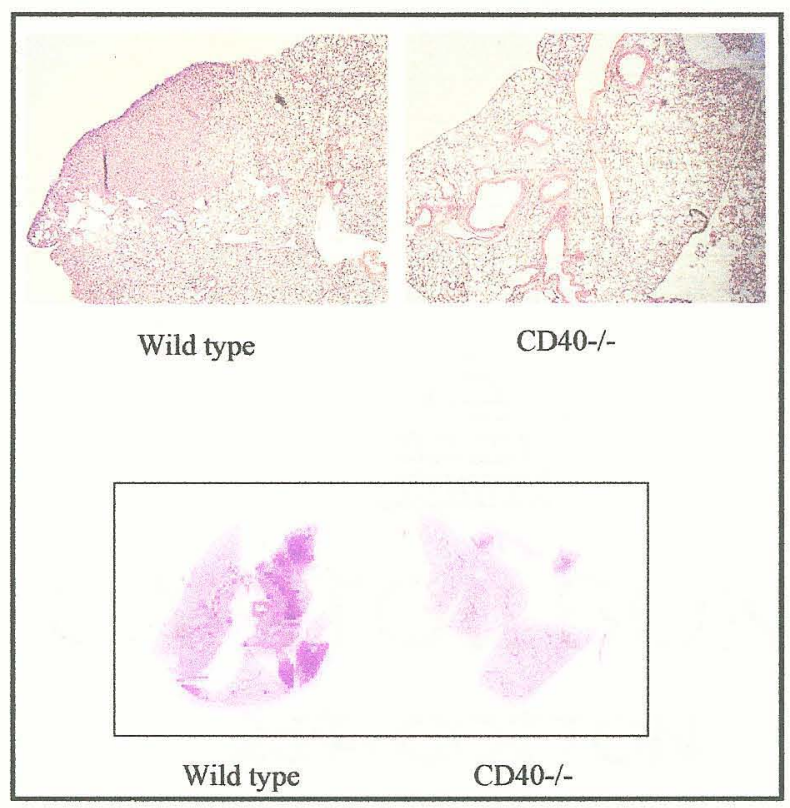

Figure 34. CD40-/- tumor bearing animals displayed reduced lung metastasis in comparison to tumor bearing wild type mice. Mice bearing $0.5 \mathrm{cc}$ subcutaneous tumors were sacrificed and purfused for lung extraction. The lungs were then sent to the University of Louisville Pathology Dept. for sectioning and an H\&E staining. Cross sections were then scanned and digital pictures were taken at 10x magnification. Images are representative of 3 mice per group. 


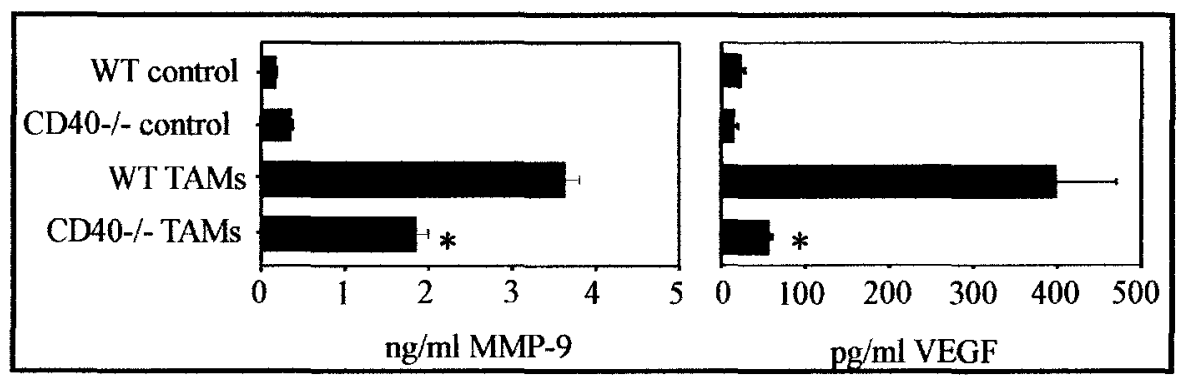

Figure 35. CD40-/- TAMs produce reduced levels of MMP-9 and VEGF in comparison to wild type TAMs. Peritoneal MФs were purified from peritoneal lavage of wild type and CD40-/-tumor bearing mice. The cells were then cultured overnight without stimulus to allow for ELISA. Data are expressed as mean \pm s.d. for 3 mice per group. ${ }^{*} p<0.05$ comparing groups wild type TAMs vs CD40-/ TAMs. Results are representative of 2 independent trials. 


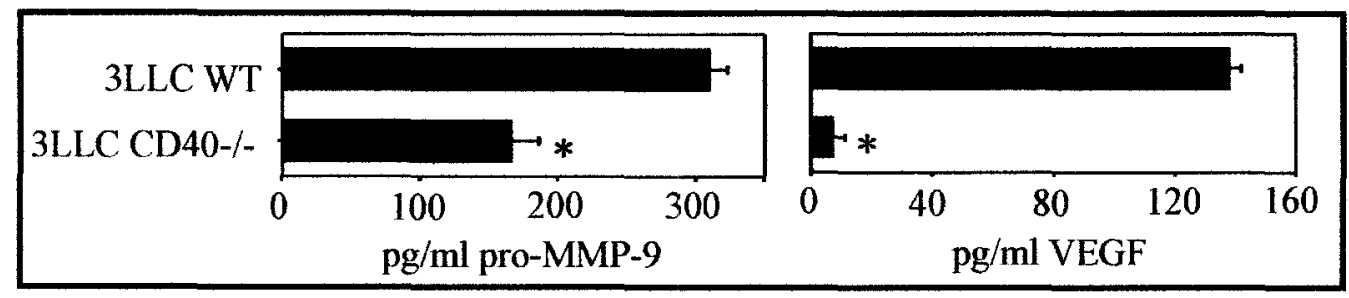

Figure 36. Tumors grown in CD40-/- mice produce significant amounts of angiogenic factors MMP-9 and VEGF, although drastically less than tumors grown in wild type mice. Tumors approaching $0.5 \mathrm{cc}$ were harvested from wild type or CD40-/- mice, made into single cell suspension and 3LLCs were purified by hMuc-1 $\mathrm{Ab}$ and magnetic beads. The cells were then plated for 17 hours to allow for cytokine accumulation without stimulus. The supernatants were then assayed by ELISA. Data are displayed as mean \pm s.d. of triplicate cultures from 3 separate mice. ${ }^{*} p<0.05$ comparing tumors from WT mice to tumor cells from CD40-/- mice. 


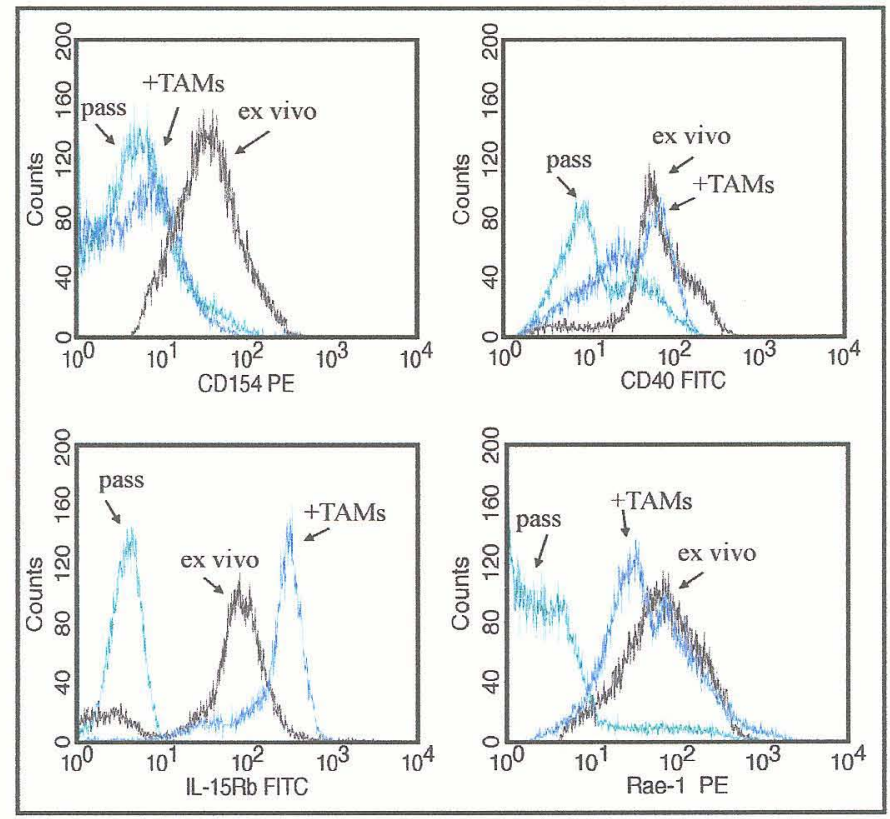

Figure 37 - TAMs restore tumor expression of surface molecules. Tumors were harvested and stained for the presence of CD154, CD40, IL-15Rb and Rae-1 ex vivo, after 3 in vitro passages and after 3 in vitro passages with TAMs added for the last $24 \mathrm{hrs}$. Results are representative of 4 independent trials. 


\begin{tabular}{|ccc|ccc|c|}
\hline 3LLC & ex vivo & pass x 2 & WT & CD40KO & IL-15KO \\
\hline CD40 & 70.4 & 4.4 & 92.8 & 6.3 & 28.9 \\
CD154 & 63.6 & 4.5 & 67.8 & 1.4 & 28.9 \\
CD44 & 148.2 & 136.5 & 325.9 & 1430.8 & 422.7 \\
\hline Rae-1 & 42.9 & 27 & 79.5 & 93.7 & 67.1 \\
NKG2D & 51.7 & 6.6 & 338.4 & 20.4 & 126.5 \\
\hline IL-15Rb & 320.1 & 7.3 & 456.7 & 10.2 & 13.4 \\
H2Kb & 26.5 & 19.5 & 317.7 & 2.65 & 413.02 \\
\hline
\end{tabular}

\begin{tabular}{|c|c|c|c|}
\hline B16F10 & ex vivo & pass X 2 & WT \\
CD40 & 11.81 & 9.59 & 11.09 \\
\hline CD154 & 8.83 & 3.78 & 9.06 \\
CD44 & 500.7 & 190.4 & 3164.8 \\
\hline Rae-1 & 56.4 & 8.9 & 63.7 \\
\hline NKG2D & 62.9 & 36.9 & 65.1 \\
\hline IL-15Rb & 98.4 & 13.8 & 609.4 \\
\hline H2Kb & 59.5 & 28.5 & 144.9 \\
\hline
\end{tabular}

\begin{tabular}{|c|cc|c|}
\hline TC-1 & ex vivo & pass x 2 & WT \\
\hline CD40 & 159.6 & 43.5 & 107.1 \\
\hline CD154 & 37.1 & 10.1 & 74.4 \\
CD44 & 706.2 & 73.06 & 1458.1 \\
\hline Rae-1 & 22.9 & 3.5 & 32.6 \\
NKG2D & 28.7 & 6.18 & 44.8 \\
\hline IL-15Rb & 7.8 & 2.5 & 4.81 \\
H2Kb & 40.9 & 3.5 & 136.7 \\
\hline
\end{tabular}

Table 1. Tumor - macrophage interactions dependent on many receptor ligand interactions, wild type TAMs, but not CD40-/- or IL-15-/- TAMs restore various tumors phenotype. Tumors were harvested and stained for the presence of CD40, CD154, CD44, Rae-1, NKG2D, IL-15Rb, and $\mathrm{H} 2 \mathrm{~Kb}$ ex vivo, after 2 in vitro passages and after 2 in vitro passages with TAMs from WT, CD40-/- and IL-15-/- mice added for the last $24 \mathrm{hrs.} \mathrm{Data}$ are displayed as mean fluorescence intensities (MFIs). Results are representative of 2 independent trials. 
restore 3LLC expression of CD44 and Rae-1 but were not able to restore 3LLC expression of CD40, CD154, NKG2D, IL-15R $\beta$, or H2Kb. In contrast, TAMs from IL- 15-/- mice effectively restored expression of CD44, NKG2D, and H2Kb, but not CD40, CD154, Rae1 or IL-15R $\beta$. In addition to surface molecule expression 3LLC cytokine production and gene expression was also greatly reduced with each in vitro passage (Fig.38). However, cytokine production and gene expression was restored or in some instances increased when 3LLCs and TAMs were co-cultured overnight (Fig 38). This apparent symbiotic relationship between TAMs and tumor cells held true for other carcinoma cell lines, such as LL-2 and TC-1 and to a smaller degree the B16F1 melanoma (Fig. 38). 


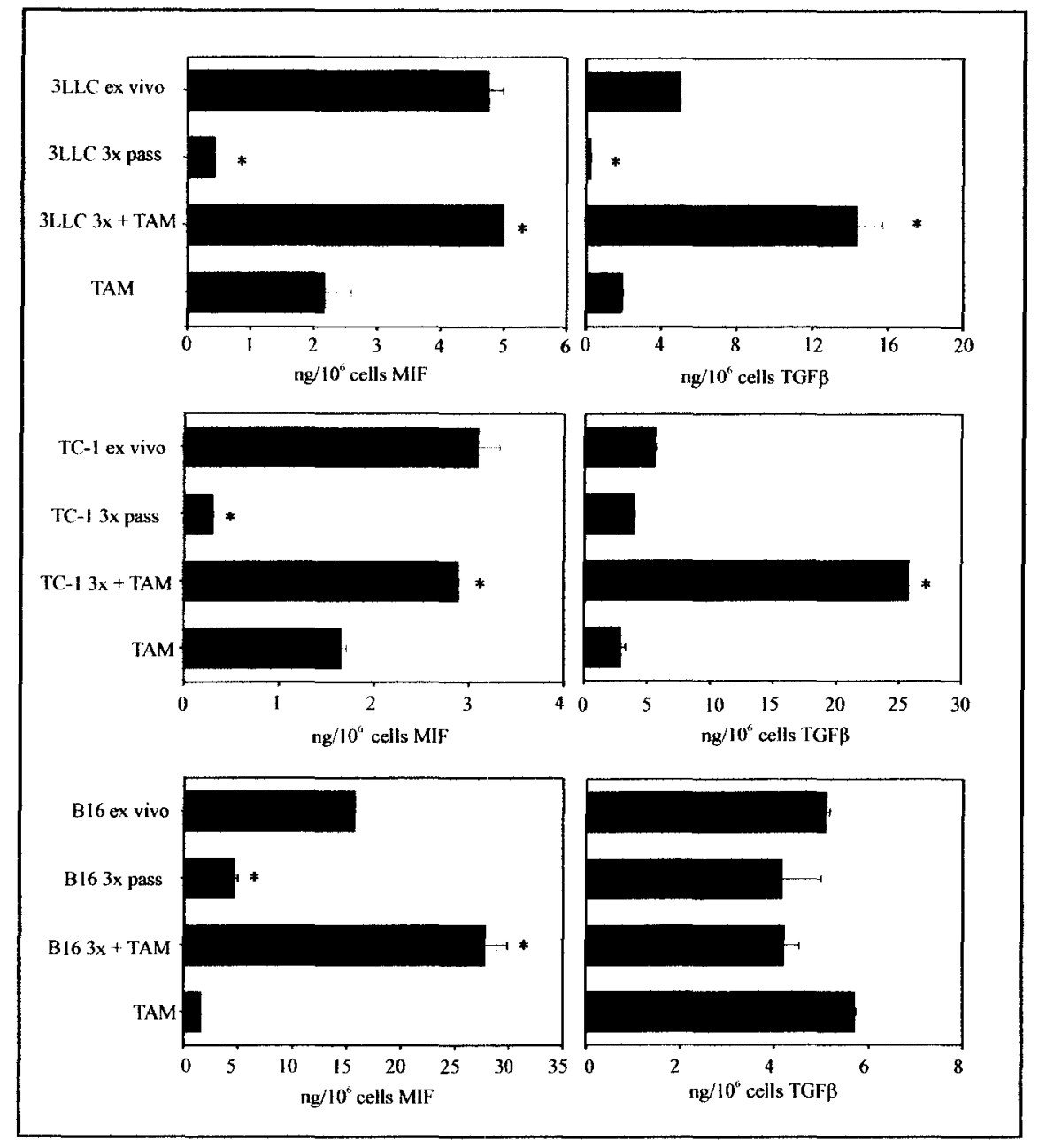

Figure 38. Tumor cytokine production induced by TAMs. Tumors were harvested and lysed for mRNA extraction for qr-PCR analysis ex vivo, after 3 in vitro passages and after 3 in vitro passages with TAMs added for the last $24 \mathrm{hrs,} \mathrm{the} \mathrm{cells} \mathrm{were} \mathrm{then} \mathrm{separated} \mathrm{prior}$ to lysis by magnetic beads. Data are displayed as ng/10 cell mRNA and mean \pm s.d. of triplicate cultures and 3 individual mice. ${ }^{*} p<0.05$ comparing ex vivo tumor cells vs in vitro passaged tumor cells or passaged tumor cells vs passaged tumor cells + TAMs. Results are representative of 3 independent trials. Results were confirmed in TC-1 carcinoma and $\mathrm{B} 16 \mathrm{~F} 1$ melanoma. 


\section{DISCUSSION}

Macrophages display an array of diverse functions which are differentially expressed based on response modifiers present in the environment. TAM function is a prime example of functional plasticity, as tumor produced metabolites alter macrophage functional properties toward tumor supportive. Tumor cells employ a number of mechanisms to prevent detection and destruction by the immune system. Mechanisms including, the down regulation of HLA class I expression on their surface, the absence of co-stimulatory molecules on tumor cells, which reduces the ability of the adaptive immune response to detect the tumor, and the production of cytokines and growth factors, which further promotes an anti-inflammatory environment ${ }^{19,20,28,42,53,95,96}$. These mechanisms can directly act on cells of the immune system by preventing their activation, for example, Ag processing is decreased on class I and class II MHC, NK cytotoxic activity, IFN $\gamma$ production and NKG2D expression are down regulated, and there is a significant increase in the number of regulatory $T$ cells ${ }^{28,67,97,98}$. The tumor and the suppressive tumor environment contributes to the altered functional phenotype and activities of macrophages 1,5,45,96. Macrophages within the tumor contribute to tumor growth by the production of pro-tumorigenic factors as shown in figure 7 and macrophages distal to the tumor mass also produce pro-tumorigenic cytokines and growth factors as shown in figures $5,7,11$, and 12. Tumor infiltrating and tumor associated macrophages, as shown here, are 
modulated by tumor produced cytokines, but are likely to receive the activation stimulus by cell contact with tumor cells or tumor exosomes.

Exosomes can bind to cells and mediate different biological functions. They were first observed to be biologically active in 1996 when B cell derived exosomes were shown to induce $\mathrm{Ag}$ specific MHC class II restricted T cell responses and also in 1998 in a report where DC derived exosomes were shown to induce tumor eradication ${ }^{99 .}$ 101. There is evidence of interaction between tumor derived exosomes and APCs. In addition to ligands, most tumor derived exosomes also have enriched heat shock proteins (HSPs), which are believed to increase interaction or uptake by APCs ${ }^{50,51}$. The exosome / APC interaction was shown to induce stimulation of an immune response, mice were injected with a single dose of plasmocytoma cell derived exosomes, and were then challenged by injection of wild type tumors. The mice undergoing challenge generated specific cytotoxic $\mathrm{T}$ cells with tumor specific immunity and were protected from tumor development ${ }^{50-52}$.

The functions that an exosome can mediate will be highly dependent on the surface proteins expressed. The surface complexes of exosomes include ligands to various cell surface receptors, which can mediate interactions between two cells. In the case of the 3LLC model used in this study, tumor cells and tumor exosomes were observed to be able to activate macrophages by ligation of CD40 and/or NKG2D as evidenced by 1) the activation of macrophages from culture with tumor released exosomes, shown in figures 21 and 22,,2) the inhibition of activation, shown in figures 25 and 26, by antiCD154 and/or anti-NKG2D, and 3) by the attenuated activation stimulation of ex vivo 
TIMs and TAMs from tumor bearing CD40-/- mice along with the reduced responsiveness of CD40-/- macrophages stimulated with exosomes as shown in figures 27 and 28 .

Taking a closer look at the impact of CD40 deficiency on tumor growth and macrophage activation, we observed a vast difference in the activation of both TAMs and tumor cells from CD40-/- tumor bearing mice. TAMs from these animals produced significantly less cytokines than TAMs harvested from wild type mice bearing tumors which were comparable in size to the CD40-/- mice. In addition to reduced macrophage activation and cytokine production, $\mathrm{CD} 40-/$ - tumor bearing mice, were not observed to have metastatic lesions on the lung, whereas the lungs of tumor bearing wild type mice contained areas of heavy metastases. Application of information from reports demonstrating that macrophages are important for metastatic spread ${ }^{7,20,31}$, and the data herein, which demonstrates that TAMs are not activated in CD40-/- tumor bearing mice, and that normal macrophages are not activated by exosomes in the presence of blocking anti-CD154, indicates that there is link between macrophage activation by CD40 ligation and tumor progression and the development of metastasis. This connection could potentially serve as a target for CD40 driven therapies. Administering an agent such as $\mathrm{CD} 154$ or anti-CD40 mAb may disrupt the tumor-macrophage relationship or the activation of tumor supportive activities in macrophages by interfering with the availability of cell contact interactions through CD40-CD154. In speculation, the disruption of this relationship may relieve some of the effects of the immuno suppressive environment on NK, DC and T cells, enabling mobilization of an anti-tumor response. Targeting CD40, by treatment with ligands and/or monoclonal Abs has previously been attempted, in the aspect of its direct effect on the tumor ${ }^{29,33,110}$. The relevance of CD40 
expression by tumors is unclear. However, literature indicates that CD40 stimulation on tumor cells, results in increased cytokine production by the tumor, most commonly IL-6 and IL-8 $93,114,118$. Both of these cytokines are important for angiogenesis and contribute to metastatic spread. CD40 activation has been shown to both induce proliferation and survival in many tumors, malignancies, and leukemias by inhibiting Fas mediated apoptosis, and has also been reported to induce growth arrest and apoptosis in several other carcinomas although, through unknown mechanisms ${ }^{29,112,114-117}$. The observed differences in stimulation of tumor cells by CD40 ligation have been attributed to the setting in which stimulus was received, for example, prolonged vs. transient stimulation and/or soluble CD154 vs. membrane bound stimulation ${ }^{29,33,93}$. The activation of CD40 has been examined in therapeutic settings by the delivery of soluble CD154 or anti-CD40 $\mathrm{mAb}$ and/or delivery of ligand through gene therapy. Reports suggest that CD154 therapy has shown promising effects, inducing elevated levels of serum IFN $\gamma$ and IL-12 as well as inducing a measurable increase in T cell activation, which can specifically recognize and destroy tumor cells ${ }^{33,110,114-116,119}$. The greatest successes acquiring DC and CD8+ T cell responses were attained by combining CD40 ligation with the administration of proinflammatory cytokines IL-12 or IL-2. CD40 stimulation can synergize with IL-2 for antitumor responses, as was shown against mouse metastatic renal cell carcinomas, the administration of combined treatment resulted in increase numbers of tumor-specific CD8+ T-cells and dendritic cells in both the spleen and liver 6, 29, 120, 121 .

In addition to CD40 ligation, in this study, we demonstrate a potential role for NKG2D ligation in tumor macrophage activation. NKG2D is an activation receptor, typically reported to be preferentially expressed by NK cells and functions, when 
activated, to allow for the detection and destruction of diseased host cells ${ }^{53}$. Furthermore, it is a multi subunit receptor complex, and NKG2D signaling is mediated by specialized signaling adaptors. Mouse NKG2D can associate with two distinct adaptors, DAP-10 and DAP-12, while human NKG2D only associates with DAP-10 ${ }^{92}$ NKG2D receptor is constitutively expressed on most innate immune effector cells including NK, TCR $\gamma \delta \mathrm{T}$ cells and most NKT cells. NKG2D expression on macrophages is controversial, it was first reported by Diefenbach in 2000, but the reports of surface expression have not been able to be confirmed, although, mRNA expression has been reported several times ${ }^{20,92,122}$. Interestingly, many reports show that there is functional NKG2D on murine IFN producing dendritic cells, which are of myeloid origin ${ }^{20,92,123,124}$. We demonstrate here that levels of NKG2D mRNA gene expression can be up or down regulated in macrophages. High NKG2D gene expression is observed in TAM populations and lower expression is observed in normal macrophages populations, which can also be up-regulated when normal macrophages are cultured in the presence of 3LLC exosomes. This data is in opposition to patterns of NKG2D expression in NK cells. Normal NK cells express high levels of NKG2D, whereas NK cells from tumor animals express lower levels of NKG2D, both mRNA as well as protein expression. Ligation of NKG2D results in surface modulation of the receptor and reduced function upon subsequent receptor engagement. Thus, chronic exposure to tumor cells expressing ligands to NKG2D alters NKG2D signaling in NK cells which facilitates the evasion of tumor cells from NK detection and cytotoxic activities $^{53}$.

Another avenue for therapy currently under investigation is cytokine therapy. Rather than targeting activation of receptors, our data supports the idea that it may be more 
beneficial to use the activation provided by the tumor and modulate the functions expressed by immune cells through cytokine treatments. TIMs and TAMs appear to be both activated and modulated by tumor cells and tumor exosomes, but they maintain a high degree of plasticity, they therefore, can continue to be rapidly converted to other functional phenotypes by the appropriate cytokine treatment. As we have shown here, TIMs and TAMs are observed to be producing tumor supportive cytokines, TGF $\beta$, VEGF, MIF, MCP-1, and IL-10, upon ex vivo analysis. However, IL-12 treatment in vitro and in vivo rapidly down-regulated or attenuated production of the tumor supportive cytokines and concomitantly up-regulated production of pro-immunogenic cytokines, TNF $\alpha$, IL-6, IL-18, and released their store of cytoplasmic IL-15. The rapid change in the functional profile suggests three things, 1) IL-12 treatment can effectively overcome the influence of the tumor environment and allows the shift of macrophage functions form pro-tumorigenic to pro-immunogenic, 2) the shift in macrophage function occurs very rapidly, at least within 90 min of IL-12 treatment, and is stable for at least up to 5 days, and 3) the shift in TAM phenotype occurred systemically as was observed by analysis of tumor infiltrating, peritoneal, lung and splenic macrophages. The importance of this shift in function, is that 1) macrophages no longer contribute to angiogenesis and tumor growth and 2) it allows for a reduction in the immunosuppressive environment, allowing NK and $\mathrm{T}$ cells to become activated. NK cells have been observed to become activated 3-5 days after IL-12 treatment and CD8 + CTLs have been observed to be present and activated 5-7 days post IL-12 treatment, both of which display cytotoxic activities and producing elevated levels of pro-inflammatory cytokines ${ }^{61,62,102-104}$. The activation and pro-inflammatory cytokine 
production of NK and CTLs, then drives the anti-tumor response, including the induction of cytotoxic and effector activities by the macrophage.

IL-12 has a strong anti-tumor effect against many cancers including melanomas and colon, renal, mammary and ovarian carcinomas ${ }^{13,60,64}$. However, enthusiasm for the use of IL-12 as a treatment for human patients, waned when i.v. administration led to severe toxicity in phase II trials ${ }^{95}$. However, toxicity has been greatly over come in recent trials by administering the cytokine by subcutaneous injections or most impressively by local delivery to the tumor in encapsulated microspheres, which allows for a slower and prolonged release of IL-12 $23,61,103,105$. A single dose of IL-12 has been shown to cause elevated levels of IFN $\gamma, \mathrm{IL}-18$, and IL-15 ${ }^{63,96,106}$. Tumor regression was consistent when elevated serum levels of IFN $\gamma$, IL-18, and IL-15 were maintained. While it has been established that NK and T cells respond to IL-12 by increasing production of IFN $\gamma$, they do not produce or release IL-15. Many cells, such as epithelia and other stromal cells, reportedly express IL-15 on their membrane, the source of IL-12 induced free IL-15 in the serum is unknown. We show here that TAMs rapidly release cytoplasmic IL-15 as a bolus, during the peak time point at which IL-15 is observed in the serum, going from undetectable to 5-6 ng/ml. This data demonstrates that TAMs are responsible, at least in part, for elevated serum IL-15.

The rapid release of IL-15 from macrophages as well as other stromal cells in response to IL-12 treatment seems to play a significant role in the efficacy of IL-12 treatment, as evidenced by the lack of lymphocyte infiltration into the tumor and metastatic lung, as well as the lack of tumor destruction and metastatic clearance when IL15 is neutralized or blocked. The data shown in this study, in the reports of IL-15 being 
essential to NK survival and activation ${ }^{76}$, the demonstration of the essential role of IL-15

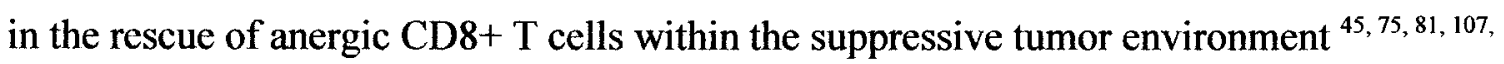
${ }^{108}$ and the observation of the ability of IL-15 to reverse tumor induced inhibition of class I MHC processing by DCs indicates that IL-15 is an essential component for effective cytokine therapy $58,71,72,74,109$. IL-15 is currently being examined as a potential candidate for cancer therapies ${ }^{69}$, however, IL-15 alone has not been shown to be as efficient as it is in combined cytokine therapies ${ }^{32,61}$. Our study indicates that TAM release of IL-15 in response to IL-12 is substantial and important to the initiation and promotion of the antitumor response . It is likely that upon release, IL-15 combines, or acts synergistically, with the IL-12, as well as other released pro-inflammatory cytokines such as IL-18, IL-21, IFN $\gamma$, etc. to promote the innate and adaptive anti-tumor responses ${ }^{61,70,84}$. According to literature there seems to be a link between available IL-15 and NKG2D expression. IL-15 has been reported to up-regulate both DAP-10 and NKG2D expression, as well as prime NKG2D signal transduction by constitutive ERK phosphorylation ${ }^{92,125-127}$. However, the mechanism of this priming remains elusive. Interestingly we observe that macrophage release of IL-15 is inhibited when NKG2D is blocked, which further indicates there may be a link between IL-15 and NKG2D expression. Smyth et. al. report that neutralization of NKG2D reversed the efficacy of IL12 treatment ${ }^{92,128}$. This report agrees with our data in two ways, 1) we show that neutralization of NKG2D prevents IL-15 release, and blocking IL-15 prevents lymphocyte infiltration into the tumor and lung, and prevents tumor destruction and metastatic clearance, and 2) neutralizing NKG2D attenuated production of other pro-inflammatory cytokines by macrophages, important for both the initiation and effector phases of the anti- 
tumor response. It is the hope that future investigations into the macrophage-tumor cell relationship will lead to better understanding of TAM activation and TAM support of tumor phenotype. Understanding of this relationship is likely to provide insight as to the mechanisms by which IL-12 induces IL-15 release by TAMs, which currently remains elusive, but is clearly an important event for effective cytokine therapy.

\section{Conclusions}

This study provides insight as to the activation and modulation of TAMs by tumor cells, tumor exosomes and tumor produced cytokines. We demonstrate that TAMs are not a stable, functional subset of suppressive tumor macrophages, but can rather can be rapidly converted to display pro-immunogenic functions by the appropriate pro-inflammatory cytokine treatment both in vitro and in vivo. Furthermore, in this demonstration we observed that macrophages are responsible, in part, for the initiation of an anti-tumor immune response by releasing a bolus of IL- 15 upon treatment with IL12. IL-15 is important to the mobilization of the NK, CD4+, and CD8+ T cells, as we demonstrated by the inhibition of mobilization when IL-15 was blocked during IL-12 treatment. As an indication of their importance in initiation of anti-tumor responses macrophages are capable of responding to IL-12 treatment, decreasing pro-tumorigenic cytokine production and increasing pro-immunogenic cytokine production and releasing intracellular IL-15, in the absence of NK cells. These functions occurred at the same rate and level whether NK cells were present or absent providing further evidence that TAMs are in an activated state, having been previously activated by the tumor, and modulate 
their function upon IL-12 treatment. Based on this study we hypothesize that it is these mechanisms by which $\mathrm{IL}-12$ is an effective treatment agent. Regardless of the therapy, it seems to first be necessary to disrupt the immuno subversive activities of the tumor, including the disruption of the tumor / macrophage relationship. This disruption provides the needed window of opportunity which allows for the generation of an antitumor cytotoxic response, and which could possibly lead to the development of central memory for long term tumor immunity. 


\section{REFERENCES}

1. Stout,R.D. \& Suttles,J. Functional plasticity of macrophages: reversible adaptation to changing microenvironments. Journal of Leukocyte Biology 76, 509-513 (2004).

2. Stout,R.D. \& Suttles,J. Immunosenescence and macrophage functional plasticity: dysregulation of macrophage function by age-associated microenvironmental changes. Immunological Reviews 205, 60-71 (2005).

3. Gordon,S. Alternative activation of macrophages. Nature Reviews Immunology 3, 23-35 (2003).

4. Arnold,L. et al. Inflammatory monocytes recruited after skeletal muscle injury switch into antiinflammatory macrophages to support myogenesis. J. Exp. Med. 204, 1057-1069 (2007).

5. Mantovani,A., Sozzani,S., Locati,M., Allavena,P., \& Sica,A. Macrophage polarization: tumor-associated macrophages as a paradigm for polarized M2 mononuclear phagocytes. Trends in Immunology 23, 549-555 (2002).

6. Bhaumik,S., Mitra,R., Varalakshmi,C., \& Khar,A. Activated macrophages migrate to the subcutaneous tumor site via the peritoneum: A novel route of cell trafficking. Experimental Cell Research 266, 44-52 (2001).

7. Allavena,P. et al. IL-10 prevents the differentiation of monocytes to dendritic cells but promotes their maturation to macrophages. European Journal of Immunology 28, 359-369 (1998). 
8. Mottonen,M. et al. Interleukin-10 inhibits the capacity of synovial macrophages to function as antigen-presenting cells. British Journal of Rheumatology 37, 1207-1214 (1998).

9. Mazzoni,A. et al. Myeloid suppressor lines inhibit T cell responses by an NOdependent mechanism. Journal of Immunology 168, 689-695 (2002).

10. Mannel,D.N. et al. Tumor-induced tumor necrosis factor production in macrophages. Lymphokine Res. 9, 485-489 (1990).

11. Stout,R.D. et al. Macrophages sequentially change their functional phenotype in response to changes in microenvironmental influences. Journal of Immunology 175, 342349 (2005).

12. Stout,R.D. \& Suttles,J. T-Cell-Macrophage Cognate Interaction in the Activation of Macrophage Effector Function by Th2 Cells. Journal of Immunology 150, 5330-5337 (1993).

13. Brunda,M.J. \& Gately,M.K. Interleukin-12: potential role in cancer therapy. Important Adv. Oncol.3-18 (1995).

14. Brunda,M.J. Interleukin-12. J. Leukoc. Biol. 55, 280-288 (1994).

15. Pollard,J.W. Tumour-educated macrophages promote tumour progression and metastasis. Nat. Rev. Cancer 4, 71-78 (2004).

16. Nowicki,A. et al. Impaired tumor growth in colony-stimulating factor 1 (CSF-1)deficient, macrophage-deficient op/op mouse: evidence for a role of CSF-1-dependent macrophages in formation of tumor stroma. Int. J. Cancer 65, 112-119 (1996). 
gland development and tumor progression. J. Mammary. Gland. Biol. Neoplasia. 7, 147162 (2002).

18. Leek,R.D. \& Harris,A.L. Tumor-associated macrophages in breast cancer. $J$. Mammary. Gland. Biol. Neoplasia. 7, 177-189 (2002).

19. Almand,B. et al. Increased production of immature myeloid cells in cancer patients: A mechanism of immunosuppression in cancer. Journal of Immunology 166, 678-689 (2001).

20. Diefenbach,A., Jamieson,A.M., Liu,S.D., Shastri,N., \& Raulet,D.H. Ligands for the murine NKG2D receptor: expression by tumor cells and activation of NK cells and macrophages. Nat. Immunol. 1, 119-126 (2000).

21. Gazzaniga,S. et al. Targeting Tumor-Associated Macrophages and Inhibition of MCP-1 Reduce Angiogenesis and Tumor Growth in a Human Melanoma Xenograft. J. Invest Dermatol.(2007).

22. Stolina,M. et al. Specific inhibition of cyclooxygenase 2 restores antitumor reactivity by altering the balance of IL-10 and IL-12 synthesis. Journal of Immunology 164, 361-370 (2000).

23. Kuriakose,M.A. et al. Interleukin-12 delivered by biodegradable microspheres promotes the antitumor activity of human peripheral blood lymphocytes in a human head and neck tumor xenograft/SCID mouse model. Head Neck 22, 57-63 (2000).

24. Meyer-Siegler,K.L., Iczkowski,K.A., Leng,L., Bucala,R., \& Vera,P.L. Inhibition of macrophage migration inhibitory factor or its receptor (CD74) attenuates growth and invasion of DU-145 prostate cancer cells. J. Immunol. 177, 8730-8739 (2006).

25. Lin,E.Y. \& Pollard,J.W. Macrophages: modulators of breast cancer progression. Novartis. Found. Symp. 256, 158-168 (2004). 
26. Lin,E.Y. et al. Macrophages regulate the angiogenic switch in a mouse model of breast cancer. Cancer Res. 66, 11238-11246 (2006).

27. Bronte,V., Serafini,P., Mazzoni,A., Segal,D.M., \& Zanovello,P. L-arginine metabolism in myeloid cells controls T-lymphocyte functions. Trends in Immunology 24, 302-306 (2003).

28. Ganss,R. \& Hanahan,D. Tumor microenvironment can restrict the effectiveness of activated antitumor lymphocytes. Cancer Res. 58, 4673-4681 (1998).

29. Ohm,J.E. et al. VEGF inhibits T-cell development and may contribute to tumorinduced immune suppression. Blood 101, 4878-4886 (2003).

30. Hiratsuka,S., Watanabe,A., Aburatani,H., \& Maru,Y. Tumour-mediated upregulation of chemoattractants and recruitment of myeloid cells predetermines lung metastasis. Nat. Cell Biol. 8, 1369-1375 (2006).

31. Condeelis,J. \& Pollard,J.W. Macrophages: obligate partners for tumor cell migration, invasion, and metastasis. Cell 124, 263-266 (2006).

32. Elgert,K.D., Alleva,D.G., \& Mullins,D.W. Tumor-induced immune dysfunction: the macrophage connection. J. Leukoc. Biol. 64, 275-290 (1998).

33. Hagemann,T. \& Balkwill,F. MIFed about cancer? Gastroenterology 129, 1785$1787(2005)$.

34. Mullins,D.W., Martins,R.S., Burger,C.J., \& Elgert,K.D. Tumor cell-derived TGFbeta and IL-10 dysregulate paclitaxel-induced macrophage activation. J. Leukoc. Biol. 69, 129-137 (2001).

35. Lamagna,C., urrand-Lions,M., \& Imhof,B.A. Dual role of macrophages in tumor growth and angiogenesis. J. Leukoc. Biol. 80, 705-713 (2006). 
36. Lewis,C.E. \& Pollard,J.W. Distinct role of macrophages in different tumor microenvironments. Cancer Res. 66, 605-612 (2006).

37. Lin,E.Y. \& Pollard,J.W. Tumor-associated macrophages press the angiogenic switch in breast cancer. Cancer Res. 67, 5064-5066 (2007).

38. Leek,R.D. et al. Macrophage infiltration is associated with VEGF and EGFR expression in breast cancer. Journal of Pathology 190, 430-436 (2000).

39. Nelson,D. \& Ganss, R. Tumor growth or regression: powered by inflammation. $J$. Leukoc. Biol. 80, 685-690 (2006).

40. Wyckoff,J.B. et al. Direct visualization of macrophage-assisted tumor cell intravasation in mammary tumors. Cancer Res. 67, 2649-2656 (2007).

41. Bordin,S. \& Young,E.T. Tumor-associated macrophages as the primary source of lysozyme in the urine of mice bearing GPC-11, a transplantable reticulum cell sarcoma. $J$. Natl. Cancer Inst. 57, 827-835 (1976).

42. Bronte,V., Serafini,P., Apolloni,E., \& Zanovello,P. Tumor-induced immune dysfunctions caused by myeloid suppressor cells. Journal of Immunotherapy 24, 431-446 (2001).

43. Bronte, V. et al. Identification of a CD11b(+)/Gr-1(+)/CD31(+) myeloid progenitor capable of activating or suppressing CD8 $(+)$ T cells. Blood 96, 3838-3846 (2000).

44. Bronte,V. et al. Apoptotic death of CD8(+) T lymphocytes after immunization: Induction of a suppressive population of Mac- $1(+) / \mathrm{Gr}-1(+)$ cells. Journal of Immunology 161, 5313-5320 (1998). 
45. Gabrilovich,D.I., Velders,M.P., Sotomayor,E.M., \& Kast,W.M. Mechanism of immune dysfunction in cancer mediated by immature $\mathrm{Gr}-1(+)$ myeloid cells. Journal of Immunology 166, 5398-5406 (2001).

46. Bronte, $\mathrm{V}$. et al. IL-4-induced arginase 1 suppresses alloreactive $\mathrm{T}$ cells in tumorbearing mice. Journal of Immunology 170, 270-278 (2003).

47. Rodriguez,P.C. et al. Arginase I production in the tumor microenvironment by mature myeloid cells inhibits T-cell receptor expression and antigen-specific T-cell responses. Cancer Research 64, 5839-5849 (2004).

48. Hagemann, T. et al. Ovarian cancer cells polarize macrophages toward a tumorassociated phenotype. J. Immunol. 176, 5023-5032 (2006).

49. Johnstone,R.M., Adam,M., Hammond,J.R., Orr,L., \& Turbide,C. Vesicle formation during reticulocyte maturation. Association of plasma membrane activities with released vesicles (exosomes). J. Biol. Chem. 262, 9412-9420 (1987).

50. Gastpar,R. et al. Heat shock protein 70 surface-positive tumor exosomes stimulate migratory and cytolytic activity of natural killer cells. Cancer Res. 65, 5238-5247 (2005).

51. Segura,E., Amigorena,S., \& Thery,C. Mature dendritic cells secrete exosomes with strong ability to induce antigen-specific effector immune responses. Blood Cells Mol. Dis. 35, 89-93 (2005).

52. Hwang,I., Shen,X., \& Sprent,J. Direct stimulation of naive T cells by membrane vesicles from antigen-presenting cells: distinct roles for CD54 and B7 molecules. Proc. Natl. Acad. Sci. U. S. A 100, 6670-6675 (2003).

53. Coudert,J.D. et al. Altered NKG2D function in NK cells induced by chronic exposure to NKG2D ligand-expressing tumor cells. Blood 106, 1711-1717 (2005). 
54. Keller,S., Sanderson,M.P., Stoeck,A., \& Altevogt,P. Exosomes: from biogenesis and secretion to biological function. Immunol. Lett. 107, 102-108 (2006).

55. Abusamra,A.J. et al. Tumor exosomes expressing Fas ligand mediate CD8+ Tcell apoptosis. Blood Cells Mol. Dis. 35, 169-173 (2005).

56. Biancone,L. et al. Activation of CD40 favors the growth and vascularization of Kaposi's sarcoma. J. Immunol. 163, 6201-6208 (1999).

57. Wagner,D.H., Jr., Stout,R.D., \& Suttles,J. Role of the CD40-CD40 ligand interaction in $\mathrm{CD} 4+\mathrm{T}$ cell contact-dependent activation of monocyte interleukin-1 synthesis. Eur. J. Immunol. 24, 3148-3154 (1994).

58. Chu,C.S. et al. Tumor-associated macrophages as a source of functional dendritic cells in ovarian cancer patients. Clinical Immunology 102, 291-301 (2002).

59. Broderick, L. et al. Human CD4(+) effector memory T cells persisting in the microenvironment of lung cancer xenografts are activated by local delivery of IL-12 to proliferate, produce IFN-gamma, and eradicate tumor cells. Journal of Immunology 174, 898-906 (2005).

60. Colombo,M.P. \& Trinchieri,G. Interleukin-12 in anti-tumor immunity and immunotherapy. Cytokine Growth Factor Rev. 13, 155-168 (2002).

61. Hill,H.C. et al. Cancer immunotherapy with interleukin 12 and granulocytemacrophage colony-stimulating factor-encapsulated microspheres: Coinduction of innate and adaptive antitumor immunity and cure of disseminated disease. Cancer Research 62, 7254-7263 (2002).

62. Trinchieri,G. Interleukin-12: a cytokine at the interface of inflammation and immunity. Adv. Immunol. 70, 83-243 (1998). 
63. Lesinski,G.B. et al. IL-12 pretreatments enhance IFN-alpha-Induced janus kinaseSTAT signaling and potentiate the antitumor effects of IFN-alpha in a murine model of malignant melanoma. Journal of Immunology 172, 7368-7376 (2004).

64. Brunda,M.J. et al. Antitumor and antimetastatic activity of interleukin 12 against murine tumors. J. Exp. Med. 178, 1223-1230 (1993).

65. Losana,G. et al. IFN-gamma and IL-12 differentially regulate CC-chemokine secretion and CCR5 expression in human T lymphocytes. J. Leukoc. Biol. 72, 735-742 (2002).

66. Egilmez,N.K. et al. Cytokine immunotherapy of cancer with controlled release biodegradable microspheres in a human tumor xenograft/SCID mouse model. Cancer Immunol. Immunother. 46, 21-24 (1998).

67. Egilmez,N.K. et al. Human CD4+ effector T cells mediate indirect interleukin-12and interferon-gamma-dependent suppression of autologous HLA-negative lung tumor xenografts in severe combined immunodeficient mice. Cancer Res. 62, 2611-2617 (2002).

68. Trinchieri,G. Interleukin-12 and interferon-gamma. Do they always go together? Am. J. Pathol. 147, 1534-1538 (1995).

69. Fehniger,T.A., Cooper,M.A., \& Caligiuri,M.A. Interleukin-2 and interleukin-15: immunotherapy for cancer. Cytokine \& Growth Factor Reviews 13, 169-183 (2002).

70. French,A.R., Holroyd,E.B., Yang,L., Kim,S., \& Yokoyama,W.M. IL-18 acts synergistically with IL-15 in stimulating natural killer cell proliferation. Cytokine 35, 229234 (2006).

71. Fehniger,T.A. \& Caligiuri,M.A. Interleukin 15: biology and relevance to human disease. Blood 97, 14-32 (2001). 
72. Kuwajima,S. et al. Interleukin 15-dependent crosstalk between conventional and plasmacytoid dendritic cells is essential for CpG-induced immune activation. Nat. Immunol. 7, 740-746 (2006).

73. Ohteki,T. Critical role for IL-15 in innate immunity. Curr. Mol. Med. 2, 371-380 (2002).

74. Ohteki,T. et al. Essential roles of DC-derived IL-15 as a mediator of inflammatory responses in vivo. J. Exp. Med. 203, 2329-2338 (2006).

75. Van Belle,T. \& Grooten,J. IL-15 and IL-15R alpha in CD4(+) T cell immunity. Archivum Immunologiae et Therapiae Experimentalis 53, 115-126 (2005).

76. Cooper,M.A. et al. Interleukin 15 is required for natural killer cell survival in vivo. Blood 98, 236A (2001).

77. Koka,R. et al. Interleukin (IL)-15R[alpha]-deficient natural killer cells survive in normal but not IL-15R[alpha]-deficient mice. J. Exp. Med. 197, 977-984 (2003).

78. Doherty,T.M., Seder,R.A., \& Sher,A. Induction and regulation of IL-15 expression in murine macrophages. J. Immunol. 156, 735-741 (1996).

79. Dubois,S., Waldmann,T.A., \& Muller,J.R. ITK and IL-15 support two distinct subsets of CD8+ T cells. Proc. Natl. Acad. Sci. U. S. A 103, 12075-12080 (2006).

80. Fehniger,T.A. et al. Fatal leukemia in interleukin 15 transgenic mice follows early expansions in natural killer and memory phenotype CD8(+) T cells. Journal of Experimental Medicine 193, 219-231 (2001). 
81. Sato,N., Patel,H.J., Waldmann,T.A., \& Tagaya,Y. The IL-15/IL-15Ralpha on cell surfaces enables sustained IL-15 activity and contributes to the long survival of CD8 memory T cells. Proc. Natl. Acad. Sci. U. S. A 104, 588-593 (2007).

82. Ohteki,T., Ho,S., Suzuki,H., Mak,T.W., \& Ohashi,P.S. Role for IL-15/LL-15 receptor beta-chain in natural killer $1.1+\mathrm{T}$ cell receptor-alpha beta+ cell development. $J$. Immunol. 159, 5931-5935 (1997).

83. Klebanoff,C.A. et al. IL-15 enhances the in vivo antitumor activity of tumorreactive CD8+ T cells. Proc. Natl. Acad. Sci. U. S. A 101, 1969-1974 (2004).

84. Smeltz,R.B. Profound enhancement of the IL-12/IL-18 pathway of IFN-gamma secretion in human CD8+ memory T cell subsets via IL-15. J. Immunol. 178, 4786-4792 (2007).

85. Murphy,W.J. et al. Synergistic anti-tumor responses after administration of agonistic antibodies to CD40 and IL-2: coordination of dendritic and CD8+ cell responses. J. Immunol. 170, 2727-2733 (2003).

86. Luo,Y. et al. Targeting tumor-associated macrophages as a novel strategy against breast cancer. J. Clin. Invest 116, 2132-2141 (2006).

87. Kusmartsev,S., Nefedova,Y., Yoder,D., \& Gabrilovich,D.I. Antigen-specific inhibition of $\mathrm{CD} 8(+) \mathrm{T}$ cell response by immature myeloid cells in cancer is mediated by reactive oxygen species (vol 172,pg 989, 2004). Journal of Immunology 172, 4647 (2004).

88. Hussein,M.R. Tumour-associated macrophages and melanoma tumourigenesis: integrating the complexity. Int. J. Exp. Pathol. 87, 163-176 (2006).

89. Meyer-Siegler,K.L., Iczkowski,K.A., \& Vera,P.L. Further evidence for increased macrophage migration inhibitory factor expression in prostate cancer. $B M C$. Cancer 5, 73 (2005). 
90. Liu,C. et al. Murine mammary carcinoma exosomes promote tumor growth by suppression of NK cell function. J. Immunol. 176, 1375-1385 (2006).

91. Yu,S. et al. Tumor exosomes inhibit differentiation of bone marrow dendritic cells. J. Immunol. 178, 6867-6875 (2007).

92. Coudert,J.D. \& Held,W. The role of the NKG2D receptor for tumor immunity. Semin. Cancer Biol. 16, 333-343 (2006).

93. Wang,T. et al. Regulation of the innate and adaptive immune responses by Stat-3 signaling in tumor cells (vol 10, pg 48, 2004). Nature Medicine 10, 209 (2004).

94. Houghton,A.M. et al. Macrophage elastase (matrix metalloproteinase-12) suppresses growth of lung metastases. Cancer Res. 66, 6149-6155 (2006).

95. Gollob,J.A. et al. Phase I trial of twice-weekly intravenous interleukin 12 in patients with metastatic renal cell cancer or malignant melanoma: ability to maintain IFNgamma induction is associated with clinical response. Clin. Cancer Res. 6, 1678-1692 (2000).

96. Watkins,S.K., Egilmez,N.K., Suttles,J., \& Stout,R.D. IL-12 Rapidly Alters the Functional Profile of Tumor-Associated and Tumor-Infiltrating Macrophages In Vitro and In Vivo. J. Immunol. 178, 1357-1362 (2007).

97. Diefenbach,A. \& Raulet,D.H. The innate immune response to tumors and its role in the induction of T-cell immunity. Immunol. Rev. 188, 9-21 (2002).

98. Valenti,R. et al. Tumor-released microvesicles as vehicles of immunosuppression. Cancer Res. 67, 2912-2915 (2007). 
99. Li,X.B., Zhang,Z.R., Schluesener,H.J., \& Xu,S.Q. Role of exosomes in immune regulation. J. Cell Mol. Med. 10, 364-375 (2006).

100. Raposo,G. et al. B lymphocytes secrete antigen-presenting vesicles. $J$. Exp. Med. 183, 1161-1172 (1996).

101. Roberts,A.I. et al. NKG2D receptors induced by IL-15 costimulate CD28negative effector CTL in the tissue microenvironment. J. Immunol. 167, 5527-5530 (2001).

102. Hess,S.D. et al. Human CD4+ T cells present within the microenvironment of human lung tumors are mobilized by the local and sustained release of IL-12 to kill tumors in situ by indirect effects of IFN-gamma. J. Immunol. 170, 400-412 (2003).

103. Nair,R.E. et al. IL-12 + GM-CSF microsphere therapy induces eradication of advanced spontaneous tumors in her-2/neu transgenic mice but fails to achieve long-term cure due to the inability to maintain effector T-cell activity. J. Immunother. (1997. ) 29, 10-20 (2006).

104. Portielje,J.E. et al. Repeated administrations of interleukin (IL)-12 are associated with persistently elevated plasma levels of IL-10 and declining IFN-gamma, tumor necrosis factor-alpha, IL-6, and IL-8 responses. Clin. Cancer Res. 9, 76-83 (2003).

105. Egilmez,N.K., Jong,Y.S., Mathiowitz,E., \& Bankert,R.B. Tumor vaccination with cytokine-encapsulated microspheres. Methods Mol. Med. 75, 687-696 (2003).

106. Gollob,J.A., Mier,J.W., \& Atkins,M.B. Clinical use of systemic IL-12 therapy. Cancer Chemother. Biol. Response Modif. 19, 353-369 (2001).

107. Teague,R.M. et al. Interleukin-15 rescues tolerant CD8+ T cells for use in adoptive immunotherapy of established tumors. Nat. Med. 12, 335-341 (2006). 
108. Diab,A., Cohen,A.D., Alpdogan,O., \& Perales,M.A. IL-15: targeting CD8+ T cells for immunotherapy. Cytotherapy. 7, 23-35 (2005).

109. Fehniger,T.A. et al. Cutting edge: IL-15 costimulates the generalized Shwartzman reaction and innate immune IFN-gamma production in vivo. Journal of Immunology 164, 1643-1647 (2000).

110. Alexandroff,A.B. et al. Role for CD40-CD40 ligand interactions in the immune response to solid tumours. Mol. Immunol. 37, 515-526 (2000).

111. Suttles,J. et al. CD40 signaling of monocyte inflammatory cytokine synthesis through an ERK1/2-dependent pathway - A target of interleukin (IL)-4 and IL-10 antiinflammatory action. Journal of Biological Chemistry 274, 5835-5842 (1999).

112. Guiducci,C., Vicari,A.P., Sangaletti,S., Trinchieri,G., \& Colombo,M.P. Redirecting in vivo elicited tumor infiltrating macrophages and dendritic cells towards tumor rejection. Cancer Res. 65, 3437-3446 (2005).

113. Stout,R.D. \& Suttles,J. The many roles of CD40 in cell-mediated inflammatory responses. Immunol. Today 17, 487-492 (1996).

114. Shorts,L. et al. Stimulation through CD40 on mouse and human renal cell carcinomas triggers cytokine production, leukocyte recruitment, and antitumor responses that can be independent of host CD40 expression. J. Immunol. 176, 6543-6552 (2006).

115. Wang,E.S. \& Wetzler,M. Targeting CD40. Leuk. Lymphoma 48, 229-231 (2007).

116. Alexandroff,A.B. et al. Role for CD40-CD40 ligand interactions in the immune response to solid tumours. Mol. Immunol. 37, 515-526 (2000). 
117. Biancone,L. et al. Activation of CD40 favors the growth and vascularization of Kaposi's sarcoma. J. Immunol. 163, 6201-6208 (1999).

118. Georgopoulos,N.T. et al. CD40-mediated death and cytokine secretion in colorectal cancer: A potential target for inflammatory tumour cell killing. Int. J. Cancer 121, 1373-1381 (2007).

119. Shorts,L. et al. Stimulation through CD40 on mouse and human renal cell carcinomas triggers cytokine production, leukocyte recruitment, and antitumor responses that can be independent of host CD40 expression. J. Immunol. 176, 6543-6552 (2006).

120. Welniak,L.A. et al. Tumor regression by anti-CD40 and interleukin-2: role of CD40 in hematopoietic cells and organ-specific effects. Biol. Blood Marrow Transplant. 10, 534-539 (2004).

121. Murphy,W.J. et al. Synergistic anti-tumor responses after administration of agonistic antibodies to CD40 and IL-2: coordination of dendritic and CD8+ cell responses. J. Immunol. 170, 2727-2733 (2003).

122. Diefenbach,A., Hsia,J.K., Hsiung,M.Y., \& Raulet,D.H. A novel ligand for the NKG2D receptor activates NK cells and macrophages and induces tumor immunity. Eur. J. Immunol. 33, 381-391 (2003).

123. Chan,C.W. et al. Interferon-producing killer dendritic cells provide a link between innate and adaptive immunity. Nat. Med. 12, 207-213 (2006).

124. Taieb,J. et al. A novel dendritic cell subset involved in tumor immunosurveillance. Nat. Med. 12, 214-219 (2006).

125. Zitvogel,L. et al. Eradication of established murine tumors using a novel cell-free vaccine: dendritic cell-derived exosomes. Nat. Med. 4, 594-600 (1998). 
126. Sutherland,C.L. et al. ULBPs, human ligands of the NKG2D receptor, stimulate tumor immunity with enhancement by IL-15. Blood 108, 1313-1319 (2006).

127. Meresse,B. et al. Coordinated induction by IL15 of a TCR-independent NKG2D signaling pathway converts CTL into lymphokine-activated killer cells in celiac disease. Immunity. 21, 357-366 (2004).

128. Smyth,M.J. et al. NKG2D function protects the host from tumor initiation. J. Exp. Med. 202, 583-588 (2005). 


\section{CURRICULUM VITAE}

Name: $\quad$ Stephanie K. Watkins

Address: Department of Microbiology and Immunology

University of Louisville School of Medicine

319 Abraham Flexnar Way

Louisville, KY 40202

DOB: $\quad$ Kenton, OH USA

04/01/1981

SSN: $\quad 270-88-6255$

\section{Education/Training:}

B.A., Bellarmine University, Louisville, KY - 1999-2003

M.S., University of Louisville, Louisville, KY - 2003-2004

$\mathrm{PhD}$ candidate, University of Louisville, Louisville, KY - 2004 - present

\section{Research and Professional Experience:}

2002-2002 Undergraduate Internship - Princess Alexandria Hospital, University of Queensland - Brisbane, Australia. Project - Insulin-like Growth Factors in Renal Cell Carcinoma.

2002-2003 Undergraduate Research Assistant - Bellarmine University, Louisville, KY. Project -Detecting and Isolating Common Strains of Polymorphic DNA from Aspergillus.

2003-2003 Research Technician - University of Louisville, Dept. of Microbiology and Immunology (June-Aug).

2003 - Graduate Research Assistant - University of Louisville School of Medicine, Department of Microbiology and Immunology. Dissertation topic-IL-12 modulation of tumor associated macrophages. 
2003 - $\quad$ Professional Membership - Society for Leukocyte Biology (SLB)

2007 - Professional Membership - American Association of Immunologist (AAI)

Awards:

1999-2003 Monsignor Horrigan Academic Scholar, Bellarmine University

2002-2003 Undergraduate Student Research Award --NIH Grant Number P20 RR16481 from the BRIN Program of the National Center for Research Resources.

2003 - Undergraduate Research Award: Dr. Robert W. Korn Research Award, Bellarmine University.

2006-2007 Pre-doctoral Fellowship Award - Lung Health Dissertation Grant Therapeutic Targeting of Macrophages Associated with Lung Carcinoma, funded by the Kentucky Chapter of the American Lung Association.

2007 - $\quad$ Renewal - Pre-doctoral Fellowship Award - Lung Health Dissertation Grant - Therapeutic Targeting of Macrophages Associated with Lung Carcinoma, funded by the Kentucky Chapter of the American Lung Association.

\section{Peer-reviewed publications:}

Stout, R. D., Jiang, C. C., Matta, B., Tietzel, I., Watkins, S.K., and Suttles, J. (2005). Macrophages sequentially change their functional phenotype in response to changes in microenvironmental influences. Journal of Immunology 2005 Jul 1;175(1):342-9.

Watkins, S.K., Egilmez, N.K., Suttles, J., Stout, R.D. (2007). IL-12 rapidly alters the functional profile of tumor-associated and tumor-infiltrating macrophages in vitro and in vivo. Journal of Immunology 2007 Feb 1; 178(3):1357-62

\section{Published abstracts:}

Watkins, S.K., Egilmez, N.K., Stout, R.D., (2005). Functional adaptivity of tumorassociated macrophages. Journal of Leukocyte Biology Supplement. S:44. 
Watkins, S.K., Egilmez, N.K., Suttles, J., Stout, R.D., (2006) Tumor-activated macrophages release cytoplasmic IL-15 in vivo in response to IL-12 immunotherapy. Journal of Leukoctye Biology Supplement. S:77.

\section{Presentations:}

\section{Seminars}

Insulin like growth factors in renal cell carcinoma. Princess Alexandra Hospital, Brisbane, Australia. Aug. 2002

Detecting and isolating common strains of polymorphic DNA from various species of Aspergillus. Butler University, Indianapolis, IN April, 2003 and Bellarmine University, Louisville, KY. May 2003.

Functional adaptivity of tumor associated macrophages. University of Louisville, Dept. Microbiology and Immunology, Louisville, KY. December 14, 2005.

Activation and functional plasticity of tumor associated macrophages University of Louisville, Dept. Microbiology and Immunology, Louisville, KY. April 24, 2007

Activation and functional plasticity of tumor associated macrophages. NCI Frederick, Frederick MD. May 4, 2007.

\section{Posters}

Therapeutic targeting of macrophages associated with lung carcinoma. Kentucky Lung Association, Louisville KY. May 2005

Functional Adaptivity of tumor associated macrophages. Society for Leukocyte Biology, Oxford, England. September 2005.

Tumor associated macrophages release cytoplasmic IL-15 in response to IL-12 treatment. Society for Leukocyte Biology, San Antonio, TX. November 2006.

Tumor associated macrophages release cytoplasmic IL-15 in response to IL-12 treatment. Brown Cancer Center, Louisville, KY. November, 2006. - Poster Award - $3^{\text {rd }}$ place.

Tumor associated macrophages release cytoplasmic IL-15 in response to IL-12 treatment. AAI meeting, Miami, FL. May 2007. 